NBER WORKING PAPER SERIES

\title{
WHAT'S MISSING IN ENVIRONMENTAL (SELF-)MONITORING: EVIDENCE FROM STRATEGIC SHUTDOWNS OF POLLUTION MONITORS
}

\author{
Yingfei Mu \\ Edward A. Rubin \\ Eric Zou \\ Working Paper 28735 \\ http://www.nber.org/papers/w28735 \\ NATIONAL BUREAU OF ECONOMIC RESEARCH \\ 1050 Massachusetts Avenue \\ Cambridge, MA 02138 \\ April 2021, Revised May 2022
}

We thank Michael Anderson, Trudy Ann Cameron, Jonathan M.V. Davis, Eric Edwards, Dave Evans, Mary Evans (thanks to the Adopt-a-Paper program), Meredith Fowlie, Cynthia Giles, Corbett Grainger, Andreas Hagemann, Alex Hollingsworth, Nicolai Kuminoff, Shanjun Li, Julian Reif, Michelle M. Rubin, Ivan Rudik, Jay Shimshack, William Wheeler, Jianwei Xing, officials at the U.S. Environmental Protection Agency, and seminar participants at Arizona State University, ASSA Annual Meeting, CU Environmental \& Resource Economics Workshop, Jinan University, Online Summer Workshop in Environment, Energy, and Transportation Economics, Society for Benefit-Cost Analysis Annual Meeting, Symposium on Natural Resources Governance for Young Scholars, and University of California Davis for helpful comments. All errors are our own. The views expressed herein are those of the authors and do not necessarily reflect the views of the National Bureau of Economic Research.

NBER working papers are circulated for discussion and comment purposes. They have not been peer-reviewed or been subject to the review by the NBER Board of Directors that accompanies official NBER publications.

(C) 2021 by Yingfei Mu, Edward A. Rubin, and Eric Zou. All rights reserved. Short sections of text, not to exceed two paragraphs, may be quoted without explicit permission provided that full credit, including $\odot$ notice, is given to the source. 
What's Missing in Environmental (Self-)Monitoring: Evidence from Strategic Shutdowns of Pollution Monitors

Yingfei Mu, Edward A. Rubin, and Eric Zou

NBER Working Paper No. 28735

April 2021, Revised May 2022

JEL No. C12,H77,Q53

\section{ABSTRACT}

Regulators often rely on regulated entities to self-monitor compliance, potentially creating strategic incentives for endogenous monitoring. This paper builds a framework to detect whether local governments skip air pollution monitoring when they expect air quality to deteriorate. The core of our method tests whether the timing of monitor shutdowns coincides with the counties' air quality alerts - public advisories based on local governments' own pollution forecasts. Applying the method to a monitor in Jersey City, NJ, suspected of a deliberate shutdown during the 2013 "Bridgegate" traffic jam, we find a 33\%reduction of this monitor's sampling rate on pollutionalert days. Building on large-scale inference tools, we then apply the method to test more than 1,300 monitors across the U.S., finding 14 metro areas with clusters of monitors showing similar strategic behavior. We assess geometric imputation and remote-sensing technologies as potential solutions to deter future strategic monitoring.

Yingfei $\mathrm{Mu}$

Department of Economics

University of Oregon

1415 Kincaid Street

Eugene, OR 97405

yingfeim@uoregon.edu

Edward A. Rubin

Department of Economics

University of Oregon

1415 Kincaid Street

Eugene, OR 97405

edwardr@uoregon.edu
Eric Zou

Department of Economics

University of Oregon

1415 Kincaid Street

Eugene, OR 97403

and NBER

ericzou@uoregon.edu 


\section{Introduction}

When regulators face substantial monitoring requirements, they commonly ask regulated entities to monitor their own compliance. Police officers are charged with turning on/off body cameras that verify their maintenance of ethical behavior; hospital staff transcribe operation events to catalog surgeons' regulatory compliance; countries self-monitor greenhouse gas emissions to demonstrate adherence to climate commitments. This system of self-monitoring is particularly common within environmental regulation, where local entities - such as state governments and individual firms - assume the roles of both the subject of regulation and the recorder of pollution data that demonstrate compliance. Can federal regulators rely on the regulated to provide complete, representative self-monitoring data? We study this question in the context of U.S. air quality regulation, where state and local governments monitor air pollution to demonstrate compliance with federally set air quality standards. We show that state agencies' leeway to decide when (not) to monitor, combined with the ability to anticipate pollution events in the near future, results in strategic timing in monitoring activities at some locations. We begin with a motivating anecdote, followed by an econometric analysis of the general prevalence of strategic monitoring.

On September $9^{\text {th }}, 2013$, two of three lanes to the George Washington Bridge closed for five days at the toll plaza connecting Fort Lee, New Jersey and Manhattan, New York for what was initially said to be a traffic study. The event was later found to be a deliberate act of political retribution. ${ }^{1}$ Coincidentally, at the time of the Bridgegate-induced traffic jams, a nearby fine particulate matter $\left(\mathrm{PM}_{2.5}\right)$ air pollution monitor on the rooftop of the Jersey City Firehouse stopped collecting data. The monitor, placed by the state government to continuously monitor compliance with federal Clean Air Act mandates, was later found to have been inoperative for 13 days (September $6^{\text {th }}-18^{\text {th }}$ ), the longest inactive period recorded in the decade since its installation. While an investigation by the U.S. Environmental Protection Agency (EPA) blamed "equipment malfunction," 2 the timing of the incident has raised concerns that the monitor was intentionally disabled so that it would not record the spike in air pollution caused by the Bridgegate traffic jam.

This incident raises a general question of whether local officials are able to deliberately halt pollution monitoring when they anticipate the monitor will record elevated pollution levels. First, state governments have the incentive to "game." While the federal EPA sets the national air quality standards (NAAQS), states and local governments self-monitor compliance with these standards. When state governments' own monitoring indicates a lack of compliance, they bear the regulatory penalties including

\footnotetext{
${ }^{1}$ See Wikipedia, The Free Encyclopedia, s.v. "Fort Lee lane closure scandal," (accessed November 13, 2020), https://en.wikipedia.org/wiki/Fort_Lee_lane_closure_scandal

${ }^{2}$ Enck, Judith. Regional Administrator of the U.S. Environmental Protection Agency Region 2. Letter to Jeff Ruch, Executive Director of the Public Employees for Environmental Responsibility. February 28, 2014.
} 
elevated requirements of expensive emission-reduction investments. Second, state governments have the discretion to game. While the federal EPA encourages states to stick to their monitoring schedules as strictly as possible, states have significant leeway, with every monitor typically allowed to miss up to $25 \%$ of its scheduled data during each quarter. Third, state governments have the ability to game. In many states, the same agencies that carry out monitoring also run advanced air quality forecasting - providing these agencies with the best data and forecasts of air quality in the near future. Despite these concurrent factors, the current system is not set up to detect strategic monitoring. Missing days are ignored by the federal regulators, implicitly assuming that pollution levels on monitored days are equal to pollution levels on unmonitored days. This tolerance for gaps in compliance monitoring data may induce strategic timing in state and local agencies' self-monitoring activity.

We propose an econometric framework that assesses whether air pollution monitors strategically shut down to avoid sampling on high-pollution days - specifically focused on identifying individual monitors whose pattern of shutdowns suggests gaming. Our framework has three components. The first component infers the government's expectation of high-pollution events through locally issued air quality alerts. These public advisories calling for citizens to reduce outdoor activities and vehicle use are often issued when forecasts predict that air pollution will exceed the Clean Air Act standards. We use an event study to assess whether a monitor's sampling rate falls when pollution alerts are in place. As a motivating example, our analysis begins with the sampling patterns from the $\mathrm{PM}_{2.5}$ monitor at the Jersey City Firehouse (JCF). Our analysis focuses on the JCF monitor's data capture rate: the share of scheduled monitoring days in which the monitor produces readings. Analyzing 21 alerts sent by Jersey City from 2007 to 2014, we show that the data capture rate of the JCF monitor drops significantly during pollution alert weeks (declining by 10 percentage points from a mean of $88 \%$ ) and especially during the alert day itself (declining by 28 percentage points). Though we do not directly address the reasons for the failure of the JCF air pollution monitor during the Bridgegate incident, our analysis indicates that the JCF monitor's sampling pattern over the seven-year period is consistent with strategic shutdowns during times of high pollution.

The second component of our framework incorporates simultaneous inference. We repeat the Jersey City Firehouse monitoring exercise to analyze 1,359 monitors that are set up to continuously sample air quality compliance for six different pollutants $\left(\mathrm{PM}_{2.5}, \mathrm{PM}_{10}, \mathrm{O}_{3}, \mathrm{NO}_{2}, \mathrm{SO}_{2}\right.$, and $\left.\mathrm{CO}\right)$ throughout the contiguous United States. These monitors are located in 167 counties with similar pollution alert programs. Importantly, our task is not to estimate the response of the average monitor, but instead to pinpoint which monitors are gaming the regulatory design by excluding days likely to have high pollution levels. This inference problem poses two challenges, which we address with large-scale inference tools (Efron, 2012). First, for each individual monitor, the event-study test for strategic shutdowns likely uses a small sample due to the limited number of alerts and/or short time series for the monitor. Consequently, traditional 
inference comparing the test statistic with its theoretical (asymptotic) null distribution is likely invalid. We remedy this issue with a randomization inference scheme, which allows us to generate an empirical null distribution based upon "placebo" event studies that each use randomly dated pollution alerts (Rosenbaum, 2002). We then calculate $p$-values, for each monitor, as the proportion of the empirical null distribution that is more extreme than the observed effect. Second, by testing a large number of monitors, there is risk of overstating the confidence of rejection for any individual monitor. We address this risk in several ways, including an assessment of the $p$-value histogram (we find an overabundance of tests with small $p$-values; see e.g., Hung, O’Neill, Bauer and Kohne, 1997; Simonsohn, Nelson, and Simmons, 2014), a standard false discovery control strategy (Benjamini and Hochberg, 1995), and an "eye-ball" screening of monitors whose patterns of strategic missingness are the most visually apparent. Following these steps, we generate a list of "interesting" monitors whose distinctive monitoring patterns warrant further regulatory attention. ${ }^{3} \mathrm{We}$ post detailed estimation results for all monitors on a publicly available website. Together, these first two steps of detection and inference offer researchers and policymakers the ability to narrow in on a potentially small subset of gamers within a much larger population of monitors/agencies.

The third component of our framework is economic characterization. We document key characteristics of these interesting monitors, and we shed light on underlying mechanisms for the patterns we see. We discover two primary features. First, we map the locations of the interesting monitors, and find 14 metro areas with clusters of interesting cases. Because the statistical procedure to determine interesting monitors does not use geographic proximity as an input, the fact that interesting cases cluster in specific regions suggests state- and/or local-government influences. Second, we use regression analysis to characterize counties with interesting monitoring patterns, and we find that a county's Clean Air Act compliance status plays a major role. For example, our state fixed effects regression suggests that being located in a noncompliant (nonattainment) county raises the probability a monitor is "interesting" by 64 percent, compared to other counties within the same state. Regressions with additional county-level characteristics, such as environmental friendliness, government size, and corruption, show limited explanatory power conditional on nonattainment status. Together, these test results support our hypothesis that strategic shutdowns arise from state and local governments' incentives to avoid or alleviate nonattainment penalties.

One possible way federal regulators could deter strategic shutdowns would be filling in missing monitoring data with values that better approximate the true air quality conditions, rather than omitting the missing days from records. We first build a $\mathrm{PM}_{2.5}$ pollution dataset with imputed values based on inverse distance weighting (IDW), a spatial-averaging prediction method commonly used in the epidemiology and

\footnotetext{
${ }^{3}$ We follow Efron (2012)'s language on the large-scale inference goal of detecting "interesting" units.
} 
the economics literatures to infer air quality at an unmonitored location using available data from nearby monitors (e.g., Shepard, 1968; Schwartz, 2001; Currie and Neidell, 2005). We adapt this idea to our study context in which data are temporally incomplete; we impute a monitor's missing value on a given day by using the inverse distance-weighted average of data from a set of nearby "donor" monitors on that day. Because donor monitors that are closer to the monitor of interest are more heavily weighted, we use a liberal, 20-mile search windows for donor monitors. This allows the IDW to provide substantial coverage while still preserving local variations in pollution concentration. We find that the IDW is able to explain $81.4 \%$ of observed $\mathrm{PM}_{2.5}$ variation and provide predictions for $38.6 \%$ of the missing values. ${ }^{4}$ In a complementary exercise, we consider an alternative imputation method that uses newly available atmospheric modelingbased $\mathrm{PM}_{2.5}$ products (Di et al., 2019) thanks to the increasing availability of satellite observations of air pollution. This second imputation is methodologically more complex, but is able to provide imputation values for all days. We use these imputed datasets to illustrate that among the aforementioned interesting monitors, the distribution of pollution on "unobserved" days exhibits a longer right tail - a pattern that replicates in both the IDW data and the modeling data. No such pattern is observed for non-interesting monitors, where the distribution of pollution across observed and unobserved days are indistinguishable from each other. In other words, although our quasi-experimental framework detects strategic monitors using a specific indicator - low levels of data capture rate around pollution alerts - these monitors turn out to the ones, and likely the only ones, that are generally strategic in sampling air quality. Had the measurements been taken for the interesting monitors, $\mathrm{PM}_{2.5}$ levels would have exceeded the $15 \mathrm{ug} / \mathrm{m}^{3}$ annual standard on $23 \%$ of these missing days and would have exceeded the $35 \mathrm{ug} / \mathrm{m}^{3}$ daily standard on $2.7 \%$ of the unmonitored days. These findings suggest strategic shutdowns could have misled federal compliance status designations. ${ }^{5} \mathrm{We}$ hope our method may provide the regulator with a tractable route to assessing strategic shutdowns beyond the scope of this study - such as monitors located in areas without pollution alert programs.

We believe that the strategic self-monitoring problem highlighted in this paper is an underappreciated challenge for environmental compliance. We reported our findings to members of the federal EPA's ambient air quality monitoring group. ${ }^{6}$ Officials with whom we spoke reacted that the shutdowns may be explained by local agencies' benevolent actions to prepare for incoming pollution

\footnotetext{
${ }^{4}$ The remaining missing observations are too far from non-missing monitors to use IDW with any confidence.

${ }^{5}$ We calculate that the forgone health values from air quality improvements that the county would otherwise have enjoyed without strategic monitoring amount to about $\$ 67$ million (2020 dollars) per strategic monitor per year

${ }^{6}$ We held a 1-hour meeting with a senior staff scientist and a statistician, both with expertise in ambient air quality monitoring and enforcement. Our discussion primarily focused on Figures 1A, 3, 4, and 5 of this paper.
} 
episodes by taking the monitors offline and conducting maintenance. ${ }^{7}$ In fact, we take away from the conversation that federal regulators tend not to worry about strategic responses in ambient air quality monitoring programs in which the entity of regulation is the state/local government - at least much less so than they would worry about point-source monitoring where the entity of regulation is often a company. The officials do agree with the importance of identifying interesting monitors. In their language, while these patterns do not necessarily suggest the local agencies are doing something "wrong", it is worth informing the corresponding agencies that their data look "different" from the data generated by others. We hope our analysis can raise awareness about monitoring and enforcement challenges associated with the tension between the imperative of national environmental protection and individual states' compliance incentives (Giles, 2020).

The existing literature on environmental federalism emphases the role of decentralization on policy decisions such as inter-regional competition of environmental standards (e.g., Oates, 2001; Levinson, 2003; Millimet, 2014). Our work contributes to an emerging literature on monitoring and enforcement (e.g., Gray and Shimshack, 2011; Shimshack, 2014; Evans and Stafford, 2019) which emphasizes the fact that federalism in environmental legislations - and in many other regulatory contexts too as we mentioned at the beginning of this paper - often comes with the decentralization of the responsibility of monitoring and enforcement as well, creating potential principle-agent type of incentive misalignment in local agencies' self-monitoring activities. We believe this paper is among the first to examine selective monitoring as local agencies' strategy to help achieve environmental compliance. We corroborate an emerging literature that reveals strategic actions that contribute to an underrepresentation of high-pollution observations in states' self-monitored air quality data in the U.S. (Fowlie, Rubin, and Walker, 2019; Sullivan and Krupnick, 2019). Examples of strategic actions include states' decisions as of where to locate pollution monitoring sites (Grainger, Schreiber, and Chang, 2017) and where to locate polluters (Morehouse and Rubin, 2021); in a related paper, Zou (2021) presents evidence of strategic polluting suppression in places where pollution monitoring follows pre-scheduled on-and-off cycles (the effect of monitoring on strategic polluting behavior). Our paper presents the converse setting in which monitors that are scheduled to operate continuously choose to strategically shut down in response to expected high pollution events (the effect of pollution on strategic monitoring behavior).

Similar phenomenon has been observed in developing country settings as well, where local officials' desire to demonstrate air quality achievements has impaired truthfulness in pollution monitoring (Andrews, 2008; Chen, Jin, Kumar, and Shi, 2012; Duflo, Greenstone, Pande, and Ryan, 2013; Duflo, Greenstone,

\footnotetext{
${ }^{7}$ We believe these explanations do not fit the data. Our findings suggest that, if anything, such maintenance actions have caused the monitors to miss out the incoming pollution peaks.
} 
Pande, and Ryan, 2018; Ghanem and Zhang, 2014; Greenstone, He, Jia, and Liu, 2020; Yang, 2020). ${ }^{8}$ Our analysis is also inspired by Bennear, Jessoe, and Olmstead (2009) who showed that, in the context of drinking water regulation, local agencies undertook more testing to avoid the appearance of violating environmental standards.

Methodologically, we demonstrate that basic econometric tools can assist in making individualized conclusions about where strategic behavior occurs. The empirical literature typically identifies the average extent of an activity and subgroup heterogeneity, but rarely seek to provide insight into exactly where "interesting" behaviors may merit a closer look. Policymakers, on the other hand, often care more about actionable evidence than of broader characterizations of the extent of a problem. Our paper aims to provide concrete evidence for regulatory responses. On this front, we are related to recent development in the application of large-scale inference tools, where the research goal is to credibly detect a relatively small group of interesting units among a sea of null (Efron, 2012). ${ }^{9}$

Section 2 provides background and a description of the data. Section 3 presents a conceptual model of strategic self-monitoring. Section 4 explains our framework and discusses the results. Section 5 describes the imputation method and the results. Section 6 concludes.

\section{Background and Data}

\subsection{Clean Air Act and Ambient Air Quality Monitoring}

The National Ambient Air Quality Standards (NAAQS). The U.S. Clean Air Act (CAA) delegates the U.S. Environmental Protection Agency (EPA) to set up safety standards in the form of maximum concentration levels for outdoor air pollution. These are the National Ambient Air Quality Standards (NAAQS). Since the 1970s, the EPA has set up NAAQS for "criteria" air pollutants including particulate matter $\left(\mathrm{PM}_{2.5}\right.$ and $\left.\mathrm{PM}_{10}\right)$, ozone $\left(\mathrm{O}_{3}\right)$, nitrogen dioxide $\left(\mathrm{NO}_{2}\right)$, sulfur dioxide $\left(\mathrm{SO}_{2}\right)$, lead $(\mathrm{Pb})$,

\footnotetext{
${ }^{8}$ A related literature analyzes emission test cheating and collusion behavior in the vehicle sector (e.g., Oliva, 2015; Reynaert, 2020; Ale-Chilet et al., 2021; Reynaert and Sallee, 2021). Our paper is also related to the broader forensic economics literature that aims at detecting hidden, socially undesirable actions, often by modeling honest behavior and testing for deviations. We are grateful for Jay Shimshack who pointed us to this strand of literature. See Zitzewitz (2012) for a review.

${ }^{9}$ See applications in bioinformatics, such as high-throughput screening for drug discovery (Malo et al., 2006), and genomics/proteomics data analysis (Dudoit, Shaffer, and Boldrick, 2003; Bantscheff et al, 2007; Huang, Sherman, and Lempicki, 2009). Within economic applications, we are most closely related to the literature on permutation inference (e.g., Barrios, Diamond, Imbens, and Kolesár, 2012; Buchmueller, Miller, and Vujicic, 2016; Young, 2016; Hagemann, 2019), multiple hypothesis testing (e.g., Anderson, 2008; Heckman et al., 2010; Finkelstein et al., 2012; Christensen and Miguel, 2018; Jones, Molitor, and Reif, 2019; List, Shaikh, and Xu, 2019; Kline and Walters, 2021) and heterogeneous treatment effects estimation (e.g., Athey and Imbens, 2016; Chernozhukov, Demirer, Duflo, and Fernandez-Val, 2018; Davis and Heller, 2020).
} 
and carbon monoxide (CO). The CAA charges state governments with monitoring air quality within their own jurisdictions. The federal EPA uses states' submitted data to categorize counties into "attainment" (adhering to the standards) and "nonattainment" (violating the standards) groups. Most criteria pollutants have two standards: a 24-hour standard and an annual standard; ozone's standard is based upon an 8-hour period. For example, a county falls into $\mathrm{PM}_{2.5}$ nonattainment if its three-year average for $\mathrm{PM}_{2.5}$ exceeds 15 $\mathrm{ug} / \mathrm{m}^{3}$, and $/$ or if the three-year average of annual $98^{\text {th }}$ percentile concentration values exceeds $35 \mathrm{ug} / \mathrm{m}^{3}$. The most updated NAAQS for all criteria pollutants are listed in the federal EPA's NAAQS Table (https://www.epa.gov/criteria-air-pollutants/naaqs-table).

Nonattainment counties face substantially elevated regulatory costs for both existing and prospective entities. The state is required to develop a State Implementation Plan (SIP) that details plantspecific regulations to bring the county back into compliance. These regulations typically involve the adoption of expensive pollution abatement technologies and emission limits on existing factories. Factories planning new production capacity in nonattainment jurisdictions must adopt technologies with the "lowest achievable emission rate," irrespective of the cost of doing so. ${ }^{10}$ Local governments and individual polluters occasionally receive direct penalties from the EPA in cases of sustained nonattainment. The NAAQS provision functions as the CAA's ultimate safeguard for outdoor air quality. Its regulatory incentives for the state economy - with respect to the compliance costs, firms' productivity changes, and labor market implications - have been widely documented in the literature (e.g., Greenstone, List, and Syverson, 2012; Walker, 2013; Blundell, Gowrisankaran, and Langer, 2018; Shapiro and Walker, 2020). A separate strand of literature finds evidence that by directing regulatory resources toward sources in high-pollution areas, local governments have been able to achieve localized air quality improvements near the violating monitors (e.g., Bento, Freedman, and Lang, 2015; Auffhammer, Bento, and Lowe, 2019).

EPA Rules for Incomplete Monitoring. To demonstrate compliance with NAAQS, states' monitoring data must satisfy completeness goals. Appendix Figure B.1 tabulates the EPA's completeness goals for each of the criteria pollutants (U.S. EPA, 2013). The typical requirement is for each monitor to take at least $75 \%$ of required samples per quarter of the year. What happens if monitoring data fall below the completeness goals? In principle, incomplete data cannot be used to demonstrate compliance, and the areas is thus designated as "unclassifiable." In practice, an unclassifiable county is treated just as an attainment county. However, if statistics computed from incomplete data suggest a potential violation of NAAQS, then the EPA can invoke rights to assign "nonattainment" status using limited data available. For

\footnotetext{
${ }^{10}$ Lowest Achievable Emission Rate, or LAER, refers to technologies that achieve the lowest possible emission rate in practice without cost consideration. In contrast, new sources in attainment jurisdictions comply with the Best Available Control Technology, which is often much less strict and allows for considerations of energy, environmental, and economic impacts and other costs.
} 
example, in the case of $\mathrm{PM}_{2.5}$ monitors, only 11 days of observations per quarter are needed for the EPA to designate violation - if, for example, the average of the available observations exceeds the annual standard of $15 \mathrm{ug} / \mathrm{m}^{3}$. If the monitor collects even fewer than 11 samples per quarter, the CAA gives the EPA the right to use alternative data. The federal regulation states that the EPA administrator "may consider factors such as monitoring site closures/moves, monitoring diligence, the consistency and levels of the daily values that are available, and nearby concentrations" in determining attainment / nonattainment status. ${ }^{11}$

These rules imply that the completeness goal per se is not subject to gaming. A violating area cannot bring itself out of nonattainment simply by reducing its data capture rate below $75 \%$ per quarter because nonattainment can be designated using very limited data (11 observations); for a non-violating area, it makes little difference if its quarterly capture rate is above the $75 \%$ level (attainment) or below (unclassifiable). However, strategic responses can arise when local monitoring agencies skip high-pollution days to water down the average (or whatever relevant statistics) of captured pollution, which is the focus of this study.

How Do Monitors Work and Why Do Monitors Miss Data? Ambient pollution monitoring involves measurement acquisition, quality assurance, and data submission. Here we briefly describe each step and explain ways in which data missingness may arise in each of these steps. Our primary reference is the EPA's Quality Assurance Handbook for Air pollution Measurement Systems (U.S. EPA, 2013).

Measurement Acquisition. The monitoring process begins with in situ ambient pollution sampling at states' monitoring sites. From the outside, a monitoring site looks like a fenced shelter; Panel A of Appendix Figure B.2 provides an example. The structures seen on the rooftop are sampling air inlets. Most of the pollution analyzers reside inside the shelter and its HVAC-controlled environment. One exception is mass filtration-based particulate pollution monitors, which are less sensitive to ambient temperature changes; these monitors are often placed directly on the rooftop. Panels B and C of Appendix Figure B.2 provide an example shelter design. The actual sampling procedure differs by the type of pollutant being monitored. For example, particulate pollution sampling $\left(\mathrm{PM}_{2.5}, \mathrm{PM}_{10}\right.$, and $\left.\mathrm{Pb}\right)$ often requires manual collection of pollution filters and subsequent laboratory analysis; trace gas (such as ozone) sampling is done with fully automated laser-based methods, and the results can be obtained on site. Monitoring fieldwork is done by state personnel or contractors with appropriate training in ambient pollution monitoring.

It is worth noting that EPA imposes stringent standards on which monitoring technologies can be used towards states' air quality monitoring. Monitors using technologies certified by the EPA (known as Federal Reference Methods or Federal Equivalence Methods) are expected to robustly operate under

1140 C.F.R. Appendix N to Part 50 - Interpretation of the National Ambient Air Quality Standards for PM2.5. 
various meteorological and pollution conditions. Pollution monitors are also not expected to "max out" at the ranges of air pollution concentrations observed in the U.S. For example, all regulatory $\mathrm{PM}_{2.5}$ monitors are capable of measuring 24-hour fine particulates mass concentration of at least $200 \mathrm{ug} / \mathrm{m}^{3}$, while over $99 \%$ of daily monitor readings in our study sample are below $100 \mathrm{ug} / \mathrm{m}^{3}$. Given these demands for regulatory monitors, there is little reason to expect a "spontaneous" relationship between monitor performance and meteorological or pollution conditions.

The missing-data problem can arise at measurement acquisition stage. Reasons for missingness may include instrument malfunction, sample contamination, preventive maintenance, staff shortage, power outage, or, as we argue in this paper, strategic non-sampling. This type of data missingness, which often lasts for only a short period of time, is likely the primary scenario of interest of this paper.

Quality Assurance. The missing-data problem can also occur if a monitor fails periodic quality control (QC) processes conducted by the state agency. For example, once every two weeks, a monitor is required to go through a one-point QC check in which the monitor is exposed to a gas of known concentration. If the test exceeds the EPA's specified critical criteria for the one-point QC check (e.g., in the case of ozone, the critical criterion is that the measured concentration exceeds the true concentration by more than 7\%), the monitoring agency voids all previous readings from that monitor, indicating that data are missing for the period extending back to the date when the monitor passed the previous one-point QC check. Similar checks are done for other instrument tasks, such as monthly flow rate audits.

It also bears mentioning how extreme values are treated in the QC process. The EPA guideline encourages manual inspections of all data to spot unusual values, which can be used to "indicate a gross error in the data collection system." Importantly for our study, an outlier is considered valid until there is an explanation for why the data should be invalidated, for example, if a subsequent one-point QC fails.

Because QC failures typically result in the invalidation of large chunks of monitoring data, this type of missingness is unlikely to explain the findings in this paper.

Data Submission. Finally, the processed and quality-controlled data are submitted by the state to the federal EPA's Air Quality System (AQS) for NAAQS compliance determination. The federal EPA has the ultimate authority to decide whether it will use a monitoring agency's submitted data in determining NAAQS compliance. Occasionally, the EPA has invalidated data submitted to the AQS after failures in federal audits. For example, in a recent incident, a contract laboratory's audit failure led data from four states to be suspended from NAAQS comparison (https://www.epa.gov/air-trends/pm25-data-omitted-airtrends-assessment). As with QC-based missingness, these cases also tend to invalidate large swaths of data. 
Consequently, these cases are also unlikely to the explain strategic, short-term missingness in monitoring data that we test in this paper.

\subsection{Pollution Alerts}

Pollution alerts are based on air quality forecasts made by state and local agencies using chemistry transport models, such as the Community Multiscale Air Quality Modeling System (CMAQ). ${ }^{12}$ An alert is issued when unfavorable local weather events (thermal inversions, light winds, high pressure zones, etc.) and emission events (traffic congestion, wildfires, etc.) are expected to push air pollution to unhealthy levels as defined by the NAAQS nonattainment standards. ${ }^{13}$ Appendix Figure B.3 shows the distribution of the predicted Air Quality Index associated with the alerts. The distribution exhibits substantial pileup at the AQI cutoff of 100 (at which point the AQI code moves from "Moderate" to "Unhealthy for Sensitive Groups") and the cutoff of 150 (at which point the AQI code becomes "Unhealthy").

We are unaware of any institutional reasons for a mechanical link between alerts and missing monitoring data. To the extent that a forecasting algorithm uses monitoring data as predictors for future pollution, lower data capture on higher pollution days, if anything, would decrease the odds of pollution alerts, generating a positive correlation between capture rate and alerts. Alerts are associated with changes in general atmospheric conditions, such as temperature and precipitation, which could influence monitors' data capture due to equipment and/or staff performance. However, such mechanical association would affect all monitors, and it should not be specific to "interesting" monitor groups. We provide further details in Section 4.1 where we discuss the empirical strategy.

\subsection{Data}

Pollution Monitoring. Pollution monitoring data come from the EPA's Air Quality System (AQS) database. We use AQS Daily Summary Data which contain information from every monitor for each day from 2004 to 2015. A daily summary record is the aggregate of all sub-daily measurements, typically 24 hourly samples taken by the monitor. Our primary variable of interest is a monitor-by-day level indicator for missing data, i.e., none of the sub-daily measurements being available.

Pollution Alerts. We obtain air pollution alerts data through the EPA AirNow (airnow.gov) Action Day Program. Action Day provides a tracker of all air quality alert programs implemented by state and

\footnotetext{
${ }^{12} \mathrm{https}: / /$ www.epa.gov/cmaq/cmaq-models- 0

${ }^{13}$ Pollution alerts are often salient. Previous research has shown that alerts suppress outdoor activities and influence transportation choices (Neidell, 2009; Cutter and Neidell, 2009; Graff Zivin and Neidell, 2009).
} 
local agencies. ${ }^{14}$ The database we use contains a total of 33,357 pollution alerts issued by 342 jurisdictions between 2004 and 2015. An advisory is often issued one day ahead of the actual alert day. We use the alert day itself to define the timing of pollution alert events.

\section{A Model of Strategic Self-Monitoring}

\subsection{Model Setup}

In this section, we present a stylized model of self-monitoring that illustrates a regulator's difficulty in eliciting complete and/or unbiased monitoring results from a regulated entity. For the sake of discussion, we describe the model in the context of pollution regulation, although the setup may be readily applied to many other contexts in which a self-monitoring agent faces the trade-off between satisfying a regulator's data-reporting requirement and the risk of revealing violations. For instance, a police officer may face this trade-off when deciding whether to turn on the body camera (particularly when anticipating a nonnegligible probability of misconduct). Readers not interested in theoretical predictions can jump to the empirical analysis in Section 4.

We consider a setting in which a federal regulator sets a standard for pollution, and a regulated entity generates monitoring data to document its level of compliance with the standard. The regulated entity - the monitor henceforth - has private information about its compliance (e.g., the local level of pollution). Suppose there are $\mathrm{N}$ days in a sample period of which $\mathrm{n}_{\mathrm{v}} \in(0, \mathrm{~N})$ days violate the regulator's standard. The remaining $\mathrm{n}_{\mathrm{c}}=\mathrm{N}-\mathrm{n}_{\mathrm{v}}$ days comply with the standard.

For simplicity, we assume the monitor has full information about all $\mathrm{N}$ realizations - i.e., whether each day violates or complies with the standard - and it chooses which observations to reveal to the regulator. In reality, depending on the context, the monitor may act upon its expectations, for instance, through pollution forecasting. The monitor reports $\left\{r_{v}, r_{c}\right\}$ to the regulator, where $r_{v} \leq n_{v}$ and $r_{c} \leq n_{c}$ are the reported number of violating and compliant observations, respectively. Let each reported observation cost the monitor $\mathrm{c}$ to generate, so that the total cost of monitoring is $\mathrm{c} \cdot\left(\mathrm{r}_{\mathrm{v}}+\mathrm{r}_{\mathrm{c}}\right)$.

Based on the reports $\left\{r_{v}, r_{c}\right\}$, the regulator imposes two kinds of penalties on the monitor. First, the regulator charges the monitor a fine of $\mathrm{p}_{\mathrm{v}}\left(\mathrm{r}_{\mathrm{v}}\right)$ for the violating days. Second, there is a penalty for missing observations $p_{m}\left(N-r_{v}-r_{c}\right)$. The monitor's reporting problem is thus choosing $\left\{r_{v}, r_{c}\right\}$ that minimizes the total loss

\footnotetext{
${ }^{14}$ For example, this includes the "Spare the Air" program in the Bay Area of California (https://www.sparetheair.org/), and the "High Pollution Advisory" program managed by the state of Arizona (https://ein.az.gov/keywords/highpollution-advisory). A full list of programs contained in the database is here: https://www.airnow.gov/aqi/action-days/.
} 


$$
\begin{gathered}
\min _{\mathrm{r}_{\mathrm{v}}, \mathrm{r}_{\mathrm{c}}} \mathrm{p}_{\mathrm{v}}\left(\mathrm{r}_{\mathrm{v}}\right)+\mathrm{p}_{\mathrm{m}}\left(\mathrm{N}-\mathrm{r}_{\mathrm{v}}-\mathrm{r}_{\mathrm{c}}\right)+\mathrm{c} \cdot\left(\mathrm{r}_{\mathrm{v}}+\mathrm{r}_{\mathrm{c}}\right) \\
\text { s.t. } 0 \leq \mathrm{r}_{\mathrm{v}} \leq \mathrm{n}_{\mathrm{v}} \text { and } 0 \leq \mathrm{r}_{\mathrm{n}} \leq \mathrm{n}_{\mathrm{n}}
\end{gathered}
$$

We assume that $c>0$ and that both penalty functions are increasing $\left(\mathrm{p}_{\mathrm{v}}^{\prime}>0\right.$ and $\left.\mathrm{p}_{\mathrm{m}}^{\prime}>0\right)$ and convex in shape $\left(\mathrm{p}_{\mathrm{v}}^{\prime \prime}>0\right.$ and $\left.\mathrm{p}_{\mathrm{m}}^{\prime \prime}>0\right)$. The convexity assumption approximates the reality where often some degree of violations or missingness are tolerated, while large number of violations and missing data are heavily penalized. We now characterize monitor's reporting behavior depending on the properties of $\mathrm{p}_{\mathrm{v}}(\cdot), \mathrm{p}_{\mathrm{m}}(\cdot)$ and c.

Proposition 1. The monitor reports $\mathrm{r}_{\mathrm{v}}^{*}=0$ and $\mathrm{n}_{\mathrm{c}}>\mathrm{r}_{\mathrm{c}}^{*}>0$ when it is feasible to find $\mathrm{r}_{\mathrm{c}}^{*}$ such that $\mathrm{p}_{\mathrm{m}}^{\prime}\left(\mathrm{N}-\mathrm{r}_{\mathrm{c}}^{*}\right)=\mathrm{c}$.

That is, the monitor reports no violations and some (but not all) compliant observations whenever it is feasible to strike a balance between the marginal penalty for missing data and the cost of monitoring. Note that the behavior characterized in Proposition 1 will become infeasible when violating days are numerous, so that reporting only compliant days will incur large penalty for missing data. ${ }^{15}$ In these cases, one would expect to observe reporting behavior characterized by the following proposition:

Proposition 2. The monitor reports $\mathrm{n}_{\mathrm{v}}>\mathrm{r}_{\mathrm{v}}^{*}>0$ and $\mathrm{n}_{\mathrm{c}}=\mathrm{r}_{\mathrm{c}}^{*}$ when $\mathrm{p}_{\mathrm{m}}^{\prime}\left(\mathrm{n}_{\mathrm{v}}-\mathrm{r}_{\mathrm{v}}^{*}\right) \geq \mathrm{c}$, where $\mathrm{r}_{\mathrm{v}}^{*}$ is determined by $\mathrm{p}_{\mathrm{m}}^{\prime}\left(\mathrm{n}_{\mathrm{v}}-\mathrm{r}_{\mathrm{v}}^{*}\right)=\mathrm{p}_{\mathrm{v}}^{\prime}\left(\mathrm{r}_{\mathrm{v}}^{*}\right)+\mathrm{c}$.

That is, the monitor reports all compliant observations plus some (but not all) violations when the penalty for missing observations is sufficiently large relative to monitoring cost. Together, Propositions 1 and 2 suggest that, because compliant observations carry a lower penalty than violations, the monitor will report some (and perhaps all) compliant days. Only when the penalty for missing data is sufficiently large does the monitor report violations (due to many violating days or a highly convex penalty for missed observations $\mathrm{p}_{\mathrm{m}}$ ). In this case, the monitor reports all compliant days and reports violation up to the point where the marginal penalty for missing data equals the marginal cost of monitoring plus the marginal fine of an additional reported violation. Importantly, in our stylized model, monitor will not choose any solution such that $\mathrm{n}_{\mathrm{v}}>\mathrm{r}_{\mathrm{v}}^{*}>0$ and $\mathrm{n}_{\mathrm{c}}>\mathrm{r}_{\mathrm{c}}^{*}>0$ - that is, the monitor will not report violating observations before they "use up" all their compliant observations - as long as the marginal damage of reporting violation is positive $\mathrm{p}_{\mathrm{v}}^{\prime}\left(\mathrm{r}_{\mathrm{v}}^{*}\right)>0$.

Finally, we show that even if a monitor is at a point where it has to reveal violating days, it will not report all of its violating days unless the penalty for missing data is overwhelmingly large:

\footnotetext{
${ }^{15}$ This scenario also depends upon the convexity of $\mathrm{p}_{\mathrm{m}}$, e.g., if $\mathrm{p}_{\mathrm{m}}^{\prime}\left(\mathrm{n}_{\mathrm{v}}\right)>\mathrm{c}$, then the monitor will report violations.
} 
Proposition 3. Monitor will not report $\mathrm{r}_{\mathrm{v}}^{*}=\mathrm{n}_{\mathrm{v}}$ and $\mathrm{r}_{\mathrm{c}}^{*}=\mathrm{n}_{\mathrm{c}}$ unless $\mathrm{p}_{\mathrm{m}}^{\prime}(0) \geq \mathrm{c}+\mathrm{p}_{\mathrm{v}}^{\prime}\left(\mathrm{n}_{\mathrm{v}}\right)$.

The condition of Proposition 3 says that complete reporting only occurs when the marginal cost of missing any observations must overwhelm marginal operating costs and the marginal regulatory fines when all violations are reported. Unless the true data have very few violations $\left(\mathrm{n}_{\mathrm{v}} \approx 0\right)$, the assumed convexity of $\mathrm{p}_{\mathrm{v}}$ and $\mathrm{p}_{\mathrm{m}}$ functions likely rules out this possibility. In Appendix $\mathrm{B}$, we provide the full proof of the propositions and discuss all other boundary possibilities.

\subsection{Implications for Empirical Analysis}

We now describe the mapping between the theoretical propositions and our empirical analysis. Our stylized model offers two general corollaries. A first corollary is on the existence of strategic monitoring: the federal regulator in our model cannot expect complete and/or unbiased reporting. It is almost always the case that violating days are undersampled relative to compliant days: $r_{v} / n_{v}<r_{c} / n_{c}$. In other words, the reported monitoring data are biased toward compliance.

The main empirical tests for the existence of strategic monitors are presented in Sections 4.1 and 4.2. In Section 4.1, we propose an event study test of strategic monitoring behavior which examines whether a monitor shuts down more frequently during pollution alerts. In Section 4.2, we scale up this test and propose inference steps to identify a set of strategic ("interesting") monitors across the country.

The advantage of the event study design of Sections 4.1 and 4.2 is that it provides a "sharp" method to tease out strategic monitoring shutdown from spontaneous missingness. A drawback of this method is that it may only identify strategic monitoring behavior in a specific setting, i.e., monitor shutdowns around pollution alerts. In practice, not all monitors reside in counties that operate pollution alert programs; local agencies may also form expectations on future pollution based on other information sources other than pollution forecasting. In Sections 5.1 and 5.2, we provide complementary evidence which shows the quasiexperimental framework we propose in Sections 4.1 and 4.2, though focusing on specific setting of pollution alerts, is likely sufficient to identify most strategic monitors. To do this, we use geospatial imputation and atmospheric modeling approaches to provide predicted value for one of the primary air pollutants: fine particulate matter $\left(\mathrm{PM}_{2.5}\right)$. We then compare the distribution of $\mathrm{PM}_{2.5}$ values on missing days and non-missing days. Evidence suggests that the distributional deviation of "missed" $\mathrm{PM}_{2.5}$ manifests

only for the interesting monitors and not for other monitors (non-interesting monitors and untested monitors in counties that do not have alert programs). In other words, although our quasi-experimental framework detects strategic monitors using a specific indicator - low levels of data capture rate around pollution alerts 
- these monitors turn out to the ones, and likely the only ones, that are generally strategic in sampling air quality.

The second corollary of our stylized model involves the heterogeneity of strategic monitoring: for a monitor that has very few violating days to begin with $\left(\mathrm{n}_{\mathrm{v}} \approx 0\right)$, the marginal fine for reporting violating days is sufficiently small that the reporting problem becomes less strategic in nature and instead reduces to a simple tradeoff between non-reporting costs and the operating cost. Our empirical analysis tests this prediction by examining whether monitors at lower risk of violating the regulatory standards are less likely to exhibit strategic monitoring. We present heterogeneity analysis in Section 4.3, in which we correlate a monitor's "interesting" / "non-interesting" status with the history of NAAQS regulatory violation and measures of pollution abatement costs from the existing literature.

\section{Framework and Evidence}

This section describes the three components of our framework and presents our findings. We begin in Section 4.1 with the Jersey City Firehouse (JCF) $\mathrm{PM}_{2.5}$ monitor and explain how we test for strategic shutdowns for a single monitor. Section 4.2 describes the simultaneous testing problem where we scale up the exercise in Section 4.1 to all 1,359 monitors. Section 4.3 presents an econometric analysis of the characteristics of monitors that are deemed "interesting" by the testing process.

\subsection{Test of Individual Monitor: The Jersey City Firehouse Monitor as an Example}

Using an event study framework, we model the JCF monitor's "capture rate" of $\mathrm{PM}_{2.5}$ data - an indicator variable equaling " 1 " when scheduled monitoring occurs - around the timing of pollution alerts (the "events"). There are a total of 37 pollution alert days in Jersey City during our study period. Alerts are sometimes issued for several consecutive days, in which case we keep the first day of the episode to avoid overlapping windows and focus on the alert issuance effect. This leaves us with 21 pollution alert events. For each alert event, we pull the JCF monitor's operational status 30 days before and 30 days after the alert day, forming an event study dataset with 1,281 observations (21 alert events multiplied by the 61-day event study window for each alert). The estimation equation is:

$$
\text { Capture Rate } \mathrm{t}_{\mathrm{t}}=1-\mathbb{I}\left(\text { Missing } \mathrm{PM}_{2.5} \text { Data }\right)_{\mathrm{t}}=\sum_{\tau \in[-30,30]} \beta_{\tau} \cdot \mathbb{I}(\mathrm{t}=\tau)+\varepsilon_{\mathrm{t}}
$$

where $\mathbb{I}(\cdot)$ represents the indicator function. Note that the $\widehat{\beta}_{\tau}$ estimates are simply the capture rate $\tau$-day relative to the pollution alert day, averaged across all 21 events. 
Our goal is to assess whether the $\widehat{\beta}_{\tau}$ 's have lower values around $\tau=0$, i.e., a lower capture rate (more missing values) near the time when a pollution alert is issued. We specify a donut difference-inmeans estimator as our test statistic:

$$
\mathrm{T}=\frac{1}{7} \sum_{\tau \in[-3,3]} \hat{\beta}_{\tau}-\frac{1}{40} \sum_{\tau \in[-30,-11]} \hat{\beta}_{\tau}
$$

which is the average capture rate within a seven-day event window around the pollution alert day, subtracted by the average capture rate outside that window, with a seven-day buffer on each side of the event window. In Appendix Figure B.4, we show that our findings are insensitive to alternative choices of the buffer window. ${ }^{16}$ Our identification premise is the standard zero-trend assumption: under the null hypothesis that the pollution alert has no impact on the capture rate, we should have $\mathrm{T}=0$, and, alternatively, if alerts do affect the capture rate, we expect $T \neq 0$. One departure from the standard event study framework is that our treatment (pollution alert) reflects the monitoring agency's belief about future pollution, and thus the shutdown of monitors may well occur before the issuance of pollution alerts. Such anticipation underlies our choice to allow the test statistic to capture potential change in shutdown rates several days before the actual alert day.

Figure 1, panel A plots the $\widehat{\beta}_{\tau}$ coefficients for the JCF monitor. The graph features a clear drop of the monitor's capture rate around the pollution alert day. The corresponding T estimate is -0.101 , meaning the capture rate within the seven-day window around a pollution alert is 10.1 percentage points lower than the outside-window average of 88 percent (an 11.5\% reduction). Note that the largest change in the monitor's capture rate occurs on the alert day and the day before, with a 28.6 percentage points reduction (a $32.5 \%$ reduction).

An important feature of Figure 1, panel $\mathrm{A}$ is that the decline in the data capture rate began days before the actual pollution alert day. This is a pattern that we repeatedly observe among "interesting" monitors. Note that pollution not only increases on the actual alert day, but that it tends to rise leading up to the alert-day peak. Thus, the capture rate pattern is likely a consequence of forward-looking agencies

\footnotetext{
${ }^{16}$ Another potential issue with the simple difference-in-means test statistic is that it may falsely categorize monitors as strategic when a long-term shutdown occurs near the pollution alert day. For example, imagine an always-active monitor that shuts down at $\tau=0$ and remains inactive for a month. From equation (1), this event is associated with a test statistic of -7.1 percentage points. In practice, we will find that such possibility is only relevant with carbon monoxide (CO) monitors that often experience seasonal shutdowns (Section 4.2). We will show that a slightly sharpened version of the test statistic

$$
\mathrm{T}=\max \left(\frac{1}{7} \sum_{\tau \in[-3,3]} \hat{\beta}_{\tau}-\frac{1}{20} \sum_{\tau \in[-30,11]} \widehat{\beta}_{\tau}, \frac{1}{7} \sum_{\tau \in[-3,3]} \widehat{\beta}_{\tau}-\frac{1}{20} \sum_{\tau \in[11,30]} \widehat{\beta}_{\tau}\right)
$$

can successfully detect strategic monitors in the presence of seasonal shutdowns, as the null hypothesis is rejected when the capture rate around the alert day is lower than that of both the pre-alert period and the post-alert period.
} 
changing monitoring effort in anticipation of a future high-pollution episode (Malani and Reif, 2015). This pattern also suggests data absences are not mechanically linked with alert issuances (Section 2.2), in which case one would expect to see a change in capture on the alert day only.

We are now ready to conduct inference on whether $\mathrm{T}$ is statistically different from zero. In a largesample setting, we could implement a $t$-test of $\mathrm{T}=0$ via an OLS regression of Capture Rate $\mathrm{t}_{\mathrm{t}}$ on an indicator for the seven-day window around the alert day. This approach has several limitations in our setting. First, it relies on the distributional assumption that the $t$-statistic of $\mathrm{T}$ under the null hypothesis will be normally distributed $\mathrm{N}(0,1)$, which may not be true in our finite-sample setting. Second, with a small sample, the magnitude and precision of $\mathrm{T}$ could be sensitive to specification choices. Therefore, rather than relying on distributional and specification assumptions, we build on the idea that, under the (sharp) null hypothesis that pollution alerts have no effects whatsoever on the monitor's capture rate, variable T does not depend on whether a pollution alert occurs; therefore, we can generate the null distribution of $\mathrm{T}$ from the data by randomly shuffling the timing of pollution alerts. In practice, we assign 21 dates as the "placebo" alert days. We restrict the randomization so that the placebo day does not occur within one month of the true alert day. In Appendix Figure B.4, we report that our results are robust to using alternative randomization buffers such as 15 days or 7 days. We repeat the process 5,000 times, each iteration generating a placebo test statistic. We then compute a two-tail $p$-value of the observed $\mathrm{T}$ as the proportion of the null distribution that is more extreme (in absolute value) than T. Note that we employ two-tail testing, allowing T to be significant for the "wrong" sign. In Section 4.2, we will show this "wrong" tail provides us with an opportunity for sanity checks.

Figure 1, panel B reports the inference exercise. The histogram plots the empirical null distribution of $\mathrm{T}$ across 5,000 randomization of pollution alerts. The vertical solid line marks the true estimate which lies outside of the 95 percent range of the null distribution, with a two-tail $p$-value of 0.014 . Evidence thus points to a statistically significant reduction of the JCF monitor's capture rate around pollution alerts.

Causal Interpretation and "Strategic" Shutdowns. Before proceeding, we discuss the causal interpretation of the $\mathrm{T}$ estimate. Taken at face value, patterns of Figure 1 suggest evidence of selective shutdowns. That is, more missing data are occurring around pollution alerts with significant deterioration of air quality. Note that selective shutdown per se is an undesirable feature of monitoring data that is worth documenting: if missing rate is differentially higher around high-pollution alerts, the resulting monitoring data will understate the true pollution concentration. Improving continuity of monitoring near these pollution events will thus increase accuracy of the monitoring data regardless of why such selective shutdowns were occurring. 
But to interpret such selective shutdowns as strategic behavior - a term that implies intentionality - we need the assumption to hold that a local government's expectation of bad air quality causes the reduction in monitor's capture rate. In other words, the strategic interpretation relies on the identification assumption that there will be no changes in the monitor's capture rate in the absence of pollution expectation. Here we discuss two concerns for potential violation of this identification assumption.

A first concern is selection. Equation (1) may be mis-specified if monitors' capture rates and pollution alerts are both correlated with some unobserved factors. We note that the permutation inference should purge the influence of unobserved factors except for those that are systematically correlated with the timing of alert issuance. Moreover, if systemic omitted variable bias exists, it likely applies broadly to many monitors. In contrast, we report in Section 4.3 that strategic shutdowns tend to occur in regions with higher risk of violating the clean air standards. Section 4.3 also shows that interesting monitors are also more likely to miss monitoring during bad pollution years in general, not just around pollution alerts.

We also note that we have little prior reasons to expect any "spontaneous" relationship between monitors' sampling rate and socioeconomic/atmospheric conditions. As we mentioned in Section 2.1, monitoring techniques certified by the EPA have stringent technological standards and can robustly operate under various meteorological and pollution conditions. The periodic quality control procedures we described in Section 2.1 are also precisely designed to make sure monitors are functioning properly. Nevertheless, in order to assess this point more directly, we consider an exercise that tries to predict daily monitoring missingness using weather conditions. Weather is a candidate for confounding that could affect both when a monitor misses observations and when local agencies issue air alerts (e.g., if bad weather events influence the functioning of monitoring devices and, at the same time, affect polluting activities such as road traffic). For this confounding to occur, some function of weather must predict missing monitoring data. We train several flexible machine-learning (ML) models that use contemporaneous and lagged weather data to predict whether monitors missed planned observations. ${ }^{17}$ None of the weather-based ML models successfully improve upon a "null model" that predicts a region's majority class ("not missing”). In fact, the models functionally ignore the weather inputs and replicate the null model - always predicting

\footnotetext{
${ }^{17}$ Specifically, we use three different ML algorithms: (i) lasso-penalized OLS regression, (ii) lasso-penalized logistic regression, and (iii) random forest. The outcome for each model is a binary indicator for whether the monitor-day's observation is missing. The predictors include contemporaneous and lagged weather features - temperature (mean, minimum, and maximum), precipitation, dew point, pressure, visibility, wind speed, wind gust, and indicators for extreme weather events. We tune the models' hyperparameters using 5-fold cross validation and ultimately assess performance on final, held out test set. The daily weather data come from NOAA's Global Summary of the Day (GSOD) 2005-2014. We use inverse-distance weighting to estimate each monitor-day's weather based upon the monitor's distance to each of the 4,579 NOAA weather stations in the GSOD data.
} 
"not missing." This fact remains true even when we oversample missing days to control for missingness days' relative infrequency.

A deeper concern is reverse causality. Because pollution forecasts such as the CMAQ use contemporaneous monitoring data as input, one might be concerned that the natural (non-strategic) absence of monitors' data may interact with the issuance of pollution alerts in ways that could generate a reversely causal relationship between monitor shutdowns and alerts. This is unlikely the case. Note that if missing data occur randomly, then the distribution of missing pollution data should mirror that of the observed data. Thus, natural missingness should not affect forecasts or alert issuances. On the other hand, if the data capture rate does fall on high pollution days (but for reasons unrelated to pollution alerts), then one would expect a decrease in the odds of pollution alerts because fewer high-pollution days are being captured. This thus creates a positive relationship between capture rates and alerts (i.e., the lower the capture rates $\rightarrow$ the smaller likelihood for alert issuance) instead of a negative one that we find in the data.

\subsection{Simultaneous Test of All Monitors}

We now repeat the exercise in Figure 1 with all other monitors. We make the following sample restrictions: First, we restrict to monitors located in counties that have issued at least two pollution alerts during our study period. Second, we restrict to monitors that are designated to sample air quality every day. For $\mathrm{PM}_{2.5}$ and $\mathrm{PM}_{10}$ monitors, this means restricting to monitors sampling on a "1-in-1-day" basis. ${ }^{18}$ For $\mathrm{O}_{3}$, $\mathrm{NO}_{2}, \mathrm{SO}_{2}$, and $\mathrm{CO}$ monitors, seasonal monitoring is often practiced (e.g., ozone is often deemed a problem only during the summer months), and we restrict to monitor-months for which at least one day of monitoring data was available. ${ }^{19}$ Our final pool of tests includes 1,359 pollution monitors (including the Jersey City Firehouse monitor) for $\mathrm{PM}_{2.5}, \mathrm{PM}_{10}, \mathrm{O}_{3}, \mathrm{NO}_{2}, \mathrm{SO}_{2}$, and $\mathrm{CO}$ located in 167 counties operating between 2004 and 2015. We begin with a collection of null hypotheses that we test at once:

$$
\left\{\mathrm{H}_{\mathrm{i}} \text { : Monitor i's operation schedule is not affected by pollution alerts }\right\}_{\mathrm{i}=1}^{\mathrm{N}}
$$

\footnotetext{
${ }^{18}$ Particulate pollution monitoring is often done intermittently (once every three or six days) for sites that still adopt manual sampling technologies. Intermittent monitoring is typically allowed by the Environmental Protection Agency in jurisdictions that are not in immediate danger of violating the NAAQS. We identify 1-in-1-day monitors using the Air Quality System database's "required day count" field. We do not test lead $(\mathrm{Pb})$ particulate monitors because virtually all lead monitors follow an intermittent monitoring schedule.

${ }^{19}$ Gaseous pollutant monitoring uses chemiluminescent technologies, and are by default conducted continuously. Our sample selection primarily reflects the fact that monitoring seasons may differ across monitors.
} 
and the corresponding mean-difference test statistics $\left\{\mathrm{T}_{\mathrm{i}}\right\}_{\mathrm{i}=1}^{\mathrm{N}}$ analogously defined as in equation (1). For each monitor, we use randomization inference to obtain its two-tail $p$-value $\mathrm{p}_{\mathrm{i}}$ measuring the degree to which the observed $\mathrm{T}_{\mathrm{i}}$ contradicts $\mathrm{H}_{\mathrm{i}}$.

We next turn to the simultaneous testing problem. At any given chosen rejection threshold $\alpha$, the test will falsely reject the null approximately $100 \alpha \%$ of the time. With a large number of hypotheses, a substantial number of monitors will be falsely considered to be "gaming." We introduce several measures to approach this issue. First, we present the $p$-value histogram. By construction, the $p$-value histogram should feature a uniform distribution $\mathrm{U}(0,1)$ if the null hypothesis holds true (i.e., alerts have no effect on data availability) for every monitor $\mathrm{i}^{20}$ In practice, the histogram of $\left\{\mathrm{p}_{\mathrm{i}}\right\}_{\mathrm{i}=1}^{\mathrm{N}}$ is potentially a mixture of cases where the null hypothesis is true and cases where the null is false. If enough monitors are gaming, one would expect a deviation from $\mathrm{U}(0,1)$; more specifically, the $p$-value histogram would exhibit an overabundance of small $p$-values $(<0.05)$. Figure 2, panel A presents the $p$-value histogram. We see a clear spike in small $p$-values in the $\mathrm{p}<0.05$ range. When test statistics are further partitioned into $\mathrm{T}_{\mathrm{i}} \leq 0$ ("correct"-signed test statistic) and $\mathrm{T}_{\mathrm{i}}>0$ ("wrong"-signed test statistic) groups, we find that the spike in small $p$-values are driven by tests with the "correct" signs, i.e., those with drops in the capture rate, rather than increases around pollution alerts (Figure 2, panel B). Figure 2 also shows that significant cases tend to emerge at the smallest $p$-values. This pattern may be consistent with (a) strategic behavior being concentrated with extreme cases rather than a large number of monitors being "slightly" strategic, and (b) the distribution of $p$-values under the alternative hypothesis is steeply right-skewed with high statistical power (Hung, O’Neill, Bauer and Kohne, 1997).

Second, we employ the Benjamini-Hochberg procedure to control for false discovery rates (Benjamini and Hochberg, 1995). This method is closely related to the $p$-value histogram. Large $p$-values on the $p$-value histogram mostly represent observations from the null hypothesis, and thus can be used to estimate the proportion of small $p$-values that also come from the null hypothesis. More formally, we order $\left\{\mathrm{p}_{\mathrm{i}}\right\}_{\mathrm{i}=1}^{\mathrm{N}}$ in an increasing order $\mathrm{p}_{(1)} \leq \cdots \leq \mathrm{p}_{(\mathrm{N})}$, and for a choice of target false discovery rate $\alpha=0.05$, we find the largest value of $\mathrm{k}$ such that $\mathrm{p}_{(\mathrm{k})} \leq \alpha \mathrm{k} / \mathrm{N}$, and reject the null for $\mathrm{i}=1, \ldots, \mathrm{k}$. For each $\mathrm{T}_{\mathrm{i}}$, we also compute a $q$-value equals to the minimum false discovery rate that can be attained when $\mathrm{T}_{\mathrm{i}}$ is

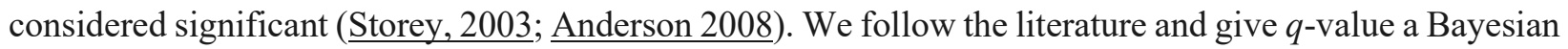
posterior significance level interpretation (i.e., false discovery adjusted significance level).

\footnotetext{
${ }^{20}$ To see this, suppose the cumulative distribution function of the test statistic $\mathrm{T}$ is $\mathrm{F}(\mathrm{t})=\operatorname{Pr}(\mathrm{T}<\mathrm{t})$. Consider a continuous and invertible $\mathrm{F}$ and a two-sided test $p$-value $=2 \min \{\operatorname{Pr}(\mathrm{T}>\mathrm{t}), \operatorname{Pr}(\mathrm{T}<\mathrm{t})\}$. Then for any $\mathrm{p} \in[0,1]$, $\operatorname{Pr}(p$-value $<\mathrm{p})=\operatorname{Pr}(2 \min \{\mathrm{F}(\mathrm{T}), 1-\mathrm{F}(\mathrm{T})\}<\mathrm{p})=\operatorname{Pr}(\min \{\mathrm{F}(\mathrm{T}), 1-\mathrm{F}(\mathrm{T})\}<\mathrm{p} / 2)=\operatorname{Pr}(\mathrm{F}(\mathrm{T})<\mathrm{p} / 2)+$ $\operatorname{Pr}(1-\mathrm{F}(\mathrm{T})<\mathrm{p} / 2)=\mathrm{p} / 2+\mathrm{p} / 2=\mathrm{p}$.
} 
Finally, recent applied econometrics has demoted sole reliance on $p$ - or $q$-values and promoted weights on the degree to which the data patterns are visually compelling. In our case, because monitoring agencies have no incentive to pull capture rates way down (Section 2.1), one would expect that interesting monitors would exhibit a T-shaped response where otherwise stable monitoring operation shows a sharp drop just around the days of high pollution episodes. To operationalize this test, we aggregate the $\widehat{\beta}_{\tau}$ 's estimates and compute average event study patterns separately for two groups: interesting monitors and other monitors. Figure 3 displays the findings. First, except for the case of $\mathrm{PM}_{10}$, the overabundance of small $p$-values is apparent for each type of monitor. Second, non-interesting monitors show a flat and stable operation pattern around pollution alerts; this suggests the average monitor is not strategically shutting down around pollution alerts. Third, except for the case of $\mathrm{CO}$, visual evidence is strong for interesting monitors, with a sharp but transient drop in capture rates around pollution alert days. As we noted, the Lshaped response for the $\mathrm{CO}$ monitors is likely driven by seasonal monitor shutdowns that are picked up by the simple difference-in-means test statistic of equation (2). In Appendix Figure B.5, we present results using a "sharpened" version of the test statistic with which the null hypothesis is rejected when the capture rate during the -3 to 3 day event window is lower than that of both the pre- and post-alert periods (Section 4.1). We find that this approach successfully identifies the T-shaped pattern for CO monitors, while the event-time patterns for other pollutants remain almost the same. For the rest of the paper, we choose to stick with the simpler test statistic as specified in equation (2).

Going one step further, we manually screen the event study patterns among all interesting monitors, and pinpoint those with "very interesting", T-shaped pattern. Figure 4 presents one example for each type of pollution monitor. Of course, this visual screening process is subjective and hence we do not use the "very interesting" status in any of the subsequent statistical analyses. We do note, however, that visual screening is likely the most directly accessible approach to regulators and practitioners in our context. We have made our estimation results for all monitors publicly available on a website. Figure 5 provides an illustration. The interactive map presents all tested areas, interesting monitors, very interesting monitors, and other tested monitors. For each monitor, we report the test statistic, the $p$ - and $q$-values, and a link to the event study graph.

\subsection{Some Features of "Interesting" Monitors}

The statistical procedure in the previous two sections generates a list of monitors whose patterns of missing data are consistent with strategic shutdowns. In this subsection, we present two exercises that document characteristics of these interesting monitors that speaks to underlying mechanisms. 
Location. Table 1 tabulates total number of pollution alerts, tested monitors, interesting monitors, and very interesting monitors by all 54 Core Based Statistical Areas (CBSAs) in our data. We find that interesting monitors tend to cluster in certain regions of the country. For example, among the 86 pollution monitors that we test in the Phoenix-Mesa-Scottsdale metro area in Arizona, 23 show up as interesting. Figure 6 maps the locations of monitors in the 14 CBSAs that in total house $60 \%$ of all these interesting monitors. The clustering pattern is not an artifact of some CBSAs simply having disproportionately more monitors. Several large metro areas we examined - such as Chicago-Naperville-Elgin (IL-IN-WI), Sacramento-Roseville-Arden-Arcade (CA), and Philadelphia-Camden-Wilmington (PA-NJ-DE-MD) have many monitors but very few interesting cases. Because the statistical procedure we use to determine interesting monitors does not use geographic proximity as an input, the fact that interesting cases cluster in certain places is informative, and suggests regional government influences.

The clustering pattern also implies that the decision to strategically monitor is spatially correlated, and some of the local variation in monitors' interesting/non-interesting status is due to the use of a sharp statistical decision criterion (i.e., monitor is interesting if its $p$-value is less than 0.05). For example, among all non-interesting $\mathrm{PM}_{2.5}$ monitors within 20 miles of interesting $\mathrm{PM}_{2.5}$ monitors, over $18 \%$ have permutation $p$-values between 0.05 and 0.15 . This fraction is $7 \%$ and $3 \%$ for non-interesting monitors within 20-50 mile and 50-100 mile distance, respectively. Put differently, some non-interesting monitors in fact do exhibit strategic monitoring patterns like their interesting neighbors, and they would have been considered as interesting if we were to use a less conservative decision rule in hypothesis testing.

County Characteristics. A key premise of our analysis is that state and local governments avoid sampling high-pollution days in an effort to either avoid nonattainment status of the federal air quality standards (NAAQS) or, in the case of counties already in violation, to move out of nonattainment. We now use cross-sectional regressions to test whether being located in counties currently in or with a history of NAAQS nonattainment in fact increased a monitor's likelihood to operate strategically. Table 2, column 1 reports a simple linear regression of an indicator variable for being labeled interesting ( $p$-value $\leq 0.05$ ) on an indicator for the NAAQS nonattainment status of the county in which the monitor is located. This is a cross-sectional regression with 1,359 underlying monitors, $11.7 \%$ of which are interesting cases. Our estimate suggests a county's nonattainment is associated with a 6.6 percentage point increase (or a $6.6 / 11.7=56$ percent increase) in the odds of the monitor being interesting. In column 2 , we repeat the "correct-vs-wrong sign" breakdown, finding that the nonattainment correlation is driven by cases with the "correct" sign (i.e., the capture rate decreases around pollution alerts). In column 3, we further control for several state-level regulatory/political characteristics including party affiliation, ${ }^{21}$ an index for

\footnotetext{
${ }^{21}$ Share of Democratic Party affiliation according to 2006 Gallup Pool.
} 
environmental friendliness, ${ }^{22}$ government size, ${ }^{23}$ and a proxy for corruption. ${ }^{24}$ We find that nonattainment is still a predominant predictor for monitor's “interesting” status. In column 4, we include state fixed effects, comparing monitors within the same state but locating in attainment versus nonattainment counties, thus purging of the influence of any observable or unobservable characteristics that might differ across states. The results again indicate a robust role of nonattainment status. In columns 5-8, we repeat the same set of regressions now using the FDR-adjusted significance, i.e., an indicator variable for $q$-value $\leq 0.05$, as the dependent variable. We obtain similar results from these alternative specifications.

Local agency's strategic monitoring behavior may also depend on how costly it is to comply with the regulation. Recent work by Shapiro and Walker (2020) provides revealed preference estimates of marginal cost of pollution abatement using price information from pollution offset trading markets that operate in nonattainment areas. These estimates are based on the fact that new polluting plants that wish to operate in nonattainment areas must offset their emissions by paying incumbent polluters to reduce their emissions; the price of these pollution offsets thus reflects the marginal pollution abatement cost in the corresponding area. We leverage the Shapiro-Walker estimates (for both NOx and VOC pollutants) to examine whether monitors in nonattainment areas facing higher marginal abatement costs are associated with a higher prevalence of strategic monitoring.

In Figure 7, panel $\mathrm{A}$, we first repeat the $p$-value distribution analysis (Figure 2) separating the sample by monitors in attainment versus nonattainment areas. Consistent with the correlate evidence of Table 2, we find that evidence of strategic monitoring (i.e., the overabundance of small $p$-values) concentrates in nonattainment areas. We then examine monitors in nonattainment areas in panel B of Figure 7, restricting to 582 monitors that located in offset trading markets. Results show that strategic monitoring mostly manifests in areas in the middle and highest terciles of the offset price distribution.

While we have focused on the importance of nonattainment history, a monitor's strategic incentive may rise as pollution levels approximate, but not yet exceed, the regulatory standards. Figure 8 provides evidence on the role of such nonattainment risk using $\mathrm{PM}_{2.5}$ monitors as an example. The chart documents the relationship between a monitor's quarterly capture rate (number of days with creditable sample as a fraction of required days of sampling) and 1-unit bins of annual $\mathrm{PM}_{2.5}$ concentration (the "design value") where an exceedance of $15 \mathrm{ug} / \mathrm{m}^{3}$ corresponds to a higher risk of violation. The underlying regression

\footnotetext{
${ }^{22}$ League of Conservation Voters score, which is based on state representatives' voting records on environmental issues. A higher score indicates to a stronger environmental preference (Dietz et al., 2015).

${ }^{23}$ Government-sector (two-digit NAICS: 92) employment as a share of total employment. Data are sourced from the Bureau of Economic Analysis.

${ }^{24}$ Per capita number of federal convictions among state and local public officials. Data are sourced from the Report to Congress on the Activities and Operations of the Public Integrity Section (Glaeser and Saks, 2006; Leeson and Sobel, 2008; Grooms, 2015).
} 
controls for monitor fixed effects and year fixed effects, so that the underlying variation comes from yearof-year changes in recorded pollution levels within the same monitor. Panel A reports that for interesting monitors, years with high levels of pollution correspond to low data capture rates. Panel B shows that for the other, non-interesting monitors, the capture rate is largely independent of the design value.

Two important messages emerge from this analysis. First, strategic monitoring may occur beyond situations that involve pollution alerts. Figure 8 panel A shows that, for interesting monitors, capture rates are generally lower when the monitor is closer to noncompliance. Second, while the identification of interesting monitors is based on the specific context of pollution alerts, the fact that Figure 8 panel B shows precise zero response from non-interesting monitors suggest the detection framework is able to capture most, if not all, $\mathrm{PM}_{2.5}$ monitors that exhibit strategic monitoring. We will see a similar set of results in Section 5.1, where we use $\mathrm{PM}_{2.5}$ imputation data to show that a deviation of pollution distribution on unobserved days from observed days exists only among the interesting monitors.

\section{Regulatory Consequences}

We now discuss potential consequences of the "interesting" (strategic) pollution monitors. We begin by estimating what the distribution of pollution readings would have been had monitoring been done on the missing days. In section 5.1, we discuss two imputation methods to achieve this goal - one uses simple geometric interpolation, and the other leverages remote sensing data and atmospheric modeling to provide pollution predictions. With the imputed data, we present evidence that the interesting monitors have the potential to distort air quality regulation, and such distortion may carry important health costs (Section 5.2). We offer some brief thoughts on the role of imputation tools in deterring strategic shutdowns in future regulation (Section 5.3).

\subsection{Imputation Methods}

The first imputation method we use is a simple and transparent prediction procedure known as the inverse distance weighting (IDW). The IDW builds on the idea that atmospheric conditions such as air pollution are often spatially correlated. The approach predicts the pollution in a given location as the average of readings from nearby "donor" monitors; each donor reading is weighted by the inverse of the donor monitor's distance to the location of interest. Formally, at any given point in time, the IDW pollution imputation for a monitor $x$ given a set of nearby donor monitors $\left\{\mathrm{x}_{\mathrm{i}}\right\}_{\mathrm{i}=1}^{\mathrm{N}}$ is 


$$
\mathrm{x}=\frac{\sum_{\mathrm{i}=1}^{\mathrm{N}}\left[\mathrm{d}\left(\mathrm{x}, \mathrm{x}_{\mathrm{i}}\right)\right]^{-1} \mathrm{x}_{\mathrm{i}}}{\sum_{\mathrm{i}=1}^{\mathrm{N}}\left[\mathrm{d}\left(\mathrm{x}, \mathrm{x}_{\mathrm{i}}\right)\right]^{-1}}
$$

where $d\left(x, x_{i}\right)$ is the distance between the monitor of interest and the donor monitor i. Because donor values that are closer to the monitor of interest are more heavily weighted, we use a liberal, 20-mile search window for donor monitors, which allows the IDW to provide substantial coverage while still preserving local variations in pollution concentration. Note that IDW "imputation" can be done even if $\mathrm{x}$ is not missing, given us an opportunity to conduct in-sample validation. The IDW is commonly used in epidemiology and environmental economics studies to improve spatial coverage of data as ground monitoring of weather and pollution is often sparse (e.g., Schwartz, 2001; Currie and Neidell, 2005). Here we adapt the same idea to the context where data are temporally incomplete.

A disadvantage of IDW is that it only works when at least one donor monitor exists within 20-mile radius to the monitor of interest. In our data, IDW provides imputation values for $38.6 \%$ of missing data. Further, because strategic monitoring behavior exhibits spatial clustering (Figure 6), one might worry the availability of donor monitors' imputation data per se is endogenous. We therefore also consider a second imputation method that relies upon machine-learned predictions of $\mathrm{PM}_{2.5}$ from Di et al. (2019) who provide predictions of daily $\mathrm{PM}_{2.5}$ concentrations for the contiguous United States on a grid of approximately $1 \mathrm{~km}$ by $1 \mathrm{~km}$ resolution. These predictions result from an ensemble of three machine-learning algorithms: a neural network, a random forest, and a boosted-tree model, each algorithm trained on more than 100 variables that should be predictive of ground-level $\mathrm{PM}_{2.5}$, including satellite-measurements of aerosol optical depth, simulation outputs from two chemical-transport models, meteorological data, physical variables like elevation, and land-use data (e.g., road density and industry). The algorithms' predictions were then aggregated in an ensemble based upon a generalized additive model. ${ }^{25} \mathrm{~A}$ pro of this second method is that it provides imputation values for all missing observations as the atmospheric modeling covers all place and time; a con is that the data result from complex modeling, thus being relatively less transparent than simple IDW. Below we present findings using these two approaches side by side.

\subsection{Regulatory and Health Implications}

Figure 9 presents results from the imputation exercise. Figure 9A presents data from the IDW method, while Figure 9B repeats the exact same exercises using the atmospheric modeling method.

\footnotetext{
${ }^{25}$ Di et al (2019) note that the ensemble step is productive because different models dominate in different parts of the country. The final cross-validated $\mathrm{R}^{2}$ for the daily $\mathrm{PM}_{2.5}$ ensemble is 0.860 with an RMSE of 2.786 .
} 
There are four panels. Each panel displays three distributions: observed $\mathrm{PM}_{2.5}$ (of course, for when monitoring is not missing), predicted $\mathrm{PM}_{2.5}$ when monitoring is not missing, and predicted $\mathrm{PM}_{2.5}$ when monitoring is missing. Hence, the two dashed lines tell us how closely the predicted $\mathrm{PM}_{2.5}$ tracks observed $\mathrm{PM}_{2.5}$ levels, and the solid line indicates what the distribution of $\mathrm{PM}_{2.5}$ would have looked like had monitoring been done on the missing days.

Take Figure 9A, left panel that summarizes data for interesting monitors using the IDW method. First, we find that IDW does a reasonable job predicting actual $\mathrm{PM}_{2.5}$ when pollution monitoring is not missing. A simple linear regression of observed $\mathrm{PM}_{2.5}$ on predicted $\mathrm{PM}_{2.5}$ yields an R-squared of 0.814 . Second, our prediction exercise suggests that, compared to observed $\mathrm{PM}_{2.5}$, the distribution of "missed" $\mathrm{PM}_{2.5}$ (solid line) features a longer right-tail. About $23.1 \%$ of the missing days would have shown $\mathrm{PM}_{2.5}$ exceeding $15 \mathrm{ug} / \mathrm{m}^{3}$ had the measurements been taken, and about $2.7 \%$ of the missing days would have exceeded $35 \mathrm{ug} / \mathrm{m}^{3}$. These fractions convert to about 6.6 days per year of annual standard exceedance and 0.8 days per year of 24-hour standard exceedance. Figure 9A, right panel shows no such discrepancy between observed and missed $\mathrm{PM}_{2.5}$ exists for non-interesting monitors. More broadly, we hope the IDW provides the regulator with a tractable tool to assess strategic shutdowns beyond the scope of this study. Evidence of Figure 9A reveals the difference in interesting monitors' $\mathrm{PM}_{2.5}$ distributions on observed days and missed days. The fact that we find the same group of monitors that respond to pollution alerts also exhibit a distribution-wide, selective pattern in the timing of absent data, suggesting that strategic monitoring goes beyond just the context of pollution alerts.

Figure 9B shows that the same patterns replicate almost exactly using the modeling data method.

Another important feature of Figure 9 is that the distributional deviation of "missed" $\mathrm{PM}_{2.5}$ manifests only for the interesting monitors and not for other monitors (which include non-interesting monitors and untested monitors in counties that do not have alert programs). In other words, although our quasi-experimental framework detects strategic monitors using a specific indicator - low levels of data capture rate around pollution alerts - these monitors turn out to the ones, and likely the only ones, that are generally strategic in sampling air quality.

Finally, we use the imputation data to provide an estimate on the health costs of strategic shutdowns. For example, how might the EPA assess the health risk imposed by an uncaptured 0.8 days per year of 24hour $\mathrm{PM}_{2.5}$ standard exceedance? We follow the idea of Sullivan and Krupnick (2018) and Fowlie, Rubin, and Walker (2019) to calculate the foregone health values due to regulation-induced air quality improvements that the county would otherwise have enjoyed without strategic monitoring. Our calculation is based on the following parameters: First, linking counts of exceedance days and county-level nonattainment history, we calculate that each additional day of 24-hour $\mathrm{PM}_{2.5}$ standard exceedance is 
correlated with a $13 \%$ increase in the odds of a county receiving a nonattainment designation in the following three years; this translates to a 9.6 percentage point increase in nonattainment probability. ${ }^{26}$ Second, to translate nonattainment status to air quality improvement, we use a published estimate that shows nonattainment causes on average $1.6 \mathrm{ug} / \mathrm{m}^{3}$ reduction in $\mathrm{PM}_{2.5}$ levels in the county per year for the 10 years following the designation (Sanders, Barreca, and Neidell, 2020). Third, to associate air quality improvement to mortality consequence, we use an epidemiology study that commonly cited by the EPA that each 10 $\mathrm{ug} / \mathrm{m}^{3}$ reduction in $\mathrm{PM}_{2.5}$ is associated with $6 \%$ reduction in all-cause adult mortality rates (Krewski et al., 2009). Finally, to convert rate changes to level changes, we use data from the Centers for Disease Control and Prevention (CDC) to calculate that the average county's baseline mortality rate in our sample is 671 per 100,000 people, and the average county's population count during the study period is 1.55 million. When multiplied together, these numbers indicate about 7.6 avoidable deaths per interesting monitor per year. Assuming a \$8.9 million Value of Statistical Life (2020 USD) commonly used by the EPA in regulatory impact analyses, these avoidable deaths amount to an annual foregone health value of $\$ 67.4$ million per interesting monitor.

\subsection{Potential Deterrence}

One implementable solution to the strategic shutdown problem is for federal regulators to change their practices regarding missing data. Rather than effectively ignoring the absence of data, regulators could use estimates to approximate conditions that prevailed during down times. Similar approaches have successfully been used in other pollution regulations. For example, one can learn from the successful enforcement experience of the EPA's Acid Rain Program (ARP), a cap-and-trade program based on a monetary system of tradeable emission allowances. All emission sources in the ARP - mostly in the powergenerating sector - are required to monitor emissions in real time through a continuous emission monitoring systems (CEMS). To incentivize monitoring compliance, the ARP specifies stringent data substitution procedures when approved CEMS technology is not used. For example, when the hourly capture rate of $\mathrm{SO}_{2}$ emissions falls below $90 \%$, the substitute data value will be the maximum value observed by looking back through the last 720 hours. The ultra-conservative approach to substitute for missing data is believed

\footnotetext{
${ }^{26}$ Specifically, we regress a dummy variable for whether the monitor's county receives a nonattainment status with respect to the 24-hour $\mathrm{PM}_{2.5}$ standard in the following three years (including this year) on the monitor's number of standard-exceeding days this year. Note that a one-day observation of 24-hour standard exceedance (over $35 \mathrm{ug} / \mathrm{m}^{3}$ on any given day) does not immediately trigger a county's nonattainment, a status that is determined by three-year moving averages of annual $98^{\text {th }}$ percentile values. Our calculation yields very similar conclusion when considering the forgone health value of 6.6 days per year of annual standard exceedance.
} 
to underly the ARP's near-perfect compliance record (Schakenbach, Vollaro, and Forte, 2006) ${ }^{27}$ The ARP's zero-tolerance approach is obviously very conservative; but it does highlight the importance of substituting for missing data - rather than ignoring missing data - in maintaining monitoring compliance. We conjecture that the type of data imputation methods we described, though imperfect, may act as a trigger for regulatory investigation, and may thus serve as reasonable deterrence to strategic shutdowns.

\section{Conclusion}

We investigate the operating patterns of air quality monitors in the U.S. using a framework that we create to test for evidence of shutdowns that are strategically timed to avoid periods when forecasts predict high levels of pollution. We identify clusters of monitors in at least 14 metropolitan areas whose patterns of operation show strong evidence of the use of such strategic timing and, thus, warrant further regulatory attention. (We make the list of these monitors available at a public website.) Our findings show that the monitors that display such operating patterns are predominantly located in federal nonattainment areas that face the likelihood of costly penalties for violations of US Clean Air Act standards.

Our work suggests that current regulatory practices that ignore gaps in compliance-monitoring data collection may incentivize strategic changes in local agencies' monitoring diligence. We propose two key ways to deter such behavior: detection and incentives. The statistical framework we have devised could detect monitors that show a pattern of skipping high-pollution days. We also suggest ways to disincentivize the use of strategic shutdowns. Regulators could consider revising the current practice of ignoring missing data when they determine compliance. For example, inverse distance weighting, a method used successfully by the research community, is one possible solution to provide imputed values. Imputation methods are imperfect, but their output may act as a trigger for regulatory investigation, and may thus serve as reasonable deterrence to strategic shutdowns.

We believe that concrete evidence can help level the playing field for environmental regulations, improve accuracy of air quality data, and motivate better design of monitoring and enforcement schemes in the future to better achieve the wider aims of improved public health from having less-polluted air. More broadly, we hope that the new possibilities made possible by large-scale inference tools can extend to other research contexts where the detection of a small group of units that evidence distinct patterns (among a sea of nulls) is important.

\footnotetext{
${ }^{27}$ See also statistics from here $<$ https://www.epa.gov/airmarkets/monitoring-insights $>$. Recent data from 2015-2019 suggests CEMS capture rate is about $99.4 \%$. We are thankful to Dr. Jeremy Schreifels at the U.S. EPA for pointing us to these data.
} 


\section{References}

Alé-Chilet, Jorge, Cuicui Chen, Jing Li, and Mathias Reynaert. "Colluding against environmental regulation." Working paper (2021).

Anderson, Michael L. "Multiple inference and gender differences in the effects of early intervention: A reevaluation of the Abecedarian, Perry Preschool, and Early Training Projects. " Journal of the American Statistical Association 103, no. 484 (2008): 1481-1495.

Andrews, Steven Q. "Inconsistencies in air quality metrics: 'Blue Sky' days and PM10 concentrations in Beijing." Environmental Research Letters 3, no. 3 (2008): 034009.

Athey, Susan, and Guido Imbens. "Recursive partitioning for heterogeneous causal effects." Proceedings of the National Academy of Sciences 113, no. 27 (2016): 7353-7360.

Auffhammer, Maximilian, Antonio M. Bento, and Scott E. Lowe. "Measuring the effects of the Clean Air Act Amendments on ambient PM10 concentrations: The critical importance of a spatially disaggregated analysis." Journal of Environmental Economics and Management 58, no. 1 (2009): 15-26.

Bantscheff, Marcus, Markus Schirle, Gavain Sweetman, Jens Rick, and Bernhard Kuster. "Quantitative mass spectrometry in proteomics: a critical review." Analytical and Bioanalytical Chemistry 389, no. 4 (2007): 1017-1031.

Bennear, Lori S., Katrina K. Jessoe, and Sheila M. Olmstead. "Sampling out: regulatory avoidance and the total coliform rule." Environmental Science \& Technology (2009): 5176-5182.

Sanders, Nicholas J., Alan I. Barreca, and Matthew J. Neidell. "Estimating Causal Effects of Particulate Matter Regulation on Mortality." Epidemiology 31, no. 2 (2020): 160-167.

Barrios, Thomas, Rebecca Diamond, Guido W. Imbens, and Michal Kolesár. "Clustering, spatial correlations, and randomization inference." Journal of the American Statistical Association 107, no. 498 (2012): 578-591.

Benjamini, Yoav, and Yosef Hochberg. "Controlling the false discovery rate: a practical and powerful approach to multiple testing." Journal of the Royal Statistical Society: Series B (Methodological) 57, no. 1 (1995): 289-300.

Bento, Antonio, Matthew Freedman, and Corey Lang. "Who benefits from environmental regulation? Evidence from the Clean Air Act Amendments." Review of Economics and Statistics 97, no. 3 (2015): 610622.

Blundell, Wesley, Gautam Gowrisankaran, and Ashley Langer. "Escalation of scrutiny: The gains from dynamic enforcement of environmental regulations." American Economic Review 110, no. 8 (2020): 255885.

Buchmueller, Thomas, Sarah Miller, and Marko Vujicic. "How do providers respond to changes in public health insurance coverage? Evidence from adult Medicaid dental benefits." American Economic Journal: Economic Policy 8, no. 4 (2016): 70-102.

Chernozhukov, Victor, Mert Demirer, Esther Duflo, and Ivan Fernandez-Val. Generic machine learning inference on heterogenous treatment effects in randomized experiments. No. w24678. National Bureau of Economic Research, 2018.

Currie, Janet, and Matthew Neidell. "Air pollution and infant health: what can we learn from California's recent experience?." The Quarterly Journal of Economics 120, no. 3 (2005): 1003-1030.

Chen, Yuyu, Ginger Zhe Jin, Naresh Kumar, and Guang Shi. "Gaming in air pollution data? Lessons from China." The BE Journal of Economic Analysis \& Policy 13, no. 3 (2012). 
Christensen, Garret, and Edward Miguel. "Transparency, reproducibility, and the credibility of economics research." Journal of Economic Literature 56, no. 3 (2018): 920-80.

Cutter, W. Bowman, and Matthew Neidell. "Voluntary information programs and environmental regulation: Evidence from 'Spare the Air'." Journal of Environmental Economics and Management 58, no. 3 (2009): 253-265.

Davis, Jonathan M.V. and Sara B. Heller. "Rethinking the Benefits of Youth Employment Programs: The Heterogeneous Effects of Summer Jobs." The Review of Economics and Statistics, 102, no. 4 (2020): 664667.

Di, Qian, Heresh Amini, Liuhua Shi, Itai Kloog, Rachel Silvern, James Kelly, M. Benjamin Sabath et al. "An ensemble-based model of PM2.5 concentration across the contiguous United States with high spatiotemporal resolution." Environment International 130 (2019): 104909.

Dietz, Thomas, Kenneth A. Frank, Cameron T. Whitley, Jennifer Kelly, and Rachel Kelly. "Political influences on greenhouse gas emissions from US states." Proceedings of the National Academy of Sciences 112, no. 27 (2015): 8254-8259.

Dudoit, Sandrine, Juliet Popper Shaffer, and Jennifer C. Boldrick. "Multiple hypothesis testing in microarray experiments." Statistical Science (2003): 71-103.

Duflo, Esther, Michael Greenstone, Rohini Pande, and Nicholas Ryan. "Truth-telling by third-party auditors and the response of polluting firms: Experimental evidence from India." The Quarterly Journal of Economics 128, no. 4 (2013): 1499-1545.

Duflo, Esther, Michael Greenstone, Rohini Pande, and Nicholas Ryan. "The value of regulatory discretion: Estimates from environmental inspections in India." Econometrica 86, no. 6 (2018): 2123-2160.

Efron, Bradley. Large-scale inference: empirical Bayes methods for estimation, testing, and prediction. Vol. 1. Cambridge University Press, 2012.

Evans, Mary F., and Sarah L. Stafford. "The Clean Air Act Watch List and federal oversight of state enforcement efforts." Journal of Environmental Economics and Management 93 (2019): 170-184.

Finkelstein, Amy, Sarah Taubman, Bill Wright, Mira Bernstein, Jonathan Gruber, Joseph P. Newhouse, Heidi Allen, Katherine Baicker, and Oregon Health Study Group. "The Oregon health insurance experiment: evidence from the first year." The Quarterly Journal of Economics 127, no. 3 (2012): 1057-1106.

Fowlie, Meredith, Edward Rubin, and Reed Walker. "Bringing satellite-based air quality estimates down to earth." In AEA Papers and Proceedings, vol. 109, pp. 283-88. 2019.

Ghanem, Dalia, and Junjie Zhang. "'Effortless Perfection:' Do Chinese cities manipulate air pollution data?" Journal of Environmental Economics and Management 68, no. 2 (2014): 203-225.

Giles, Cynthia. "Next Generation Compliance: Environmental Regulation for the Modern Era." Harvard Law School Environmental and Energy Law Program, Cambridge, Massachusetts (2020).

Glaeser, Edward L., and Raven E. Saks. "Corruption in America." Journal of Public Economics 90, no. 67 (2006): 1053-1072.

Graff Zivin, Joshua, and Matthew Neidell. "Days of haze: Environmental information disclosure and intertemporal avoidance behavior." Journal of Environmental Economics and Management 58, no. 2 (2009): 119-128.

Grainger, Corbett, Andrew Schreiber, and Wonjun Chang. " Do Regulators Strategically Avoid Pollution Hotspots when Siting Monitors? Evidence from Remote Sensing of Air Pollution." Working paper (2017). 
Gray, Wayne B., and Jay P. Shimshack. "The effectiveness of environmental monitoring and enforcement: A review of the empirical evidence." Review of Environmental Economics and Policy 5, no. 1 (2011): 324.

Greenstone, Michael, Guojun He, Ruixue Jia, and Tong Liu. Can Technology Solve the Principal-Agent Problem? Evidence from China's War on Air Pollution. No. w27502. National Bureau of Economic Research, 2020.

Greenstone, Michael, John A. List, and Chad Syverson. The effects of environmental regulation on the competitiveness of US manufacturing. No. w18392. National Bureau of Economic Research, 2012.

Grooms, Katherine K. "Enforcing the Clean Water Act: The effect of state-level corruption on compliance." Journal of Environmental Economics and Management 73 (2015): 50-78.

Hagemann, Andreas. "Placebo inference on treatment effects when the number of clusters is small." Journal of Econometrics 213, no. 1 (2019): 190-209.

Heckman, James, Seong Hyeok Moon, Rodrigo Pinto, Peter Savelyev, and Adam Yavitz. "Analyzing social experiments as implemented: A reexamination of the evidence from the HighScope Perry Preschool Program." Quantitative Economics 1, no. 1 (2010): 1-46.

Huang, Da Wei, Brad T. Sherman, and Richard A. Lempicki. "Bioinformatics enrichment tools: paths toward the comprehensive functional analysis of large gene lists." Nucleic Acids Research 37, no. 1 (2009): $1-13$.

Hung, HM James, Robert T. O'Neill, Peter Bauer, and Karl Kohne. "The behavior of the p-value when the alternative hypothesis is true." Biometrics (1997): 11-22.

Jones, Damon, David Molitor, and Julian Reif. "What do workplace wellness programs do? Evidence from the Illinois workplace wellness study." The Quarterly Journal of Economics 134, no. 4 (2019): 1747-1791.

Kline, Patrick, and Christopher Walters. "Reasonable Doubt: Experimental Detection of Job - Level Employment Discrimination." Econometrica 89, no. 2 (2021): 765-792.

Krewski, Daniel, Michael Jerrett, Richard T. Burnett, Renjun Ma, Edward Hughes, Yuanli Shi, Michelle C. Turner et al. Extended follow-up and spatial analysis of the American Cancer Society study linking particulate air pollution and mortality. No. 140. Boston, MA: Health Effects Institute, 2009.

Leeson, Peter T., and Russell S. Sobel. "Weathering corruption." Journal of Law and Economics 51, no. 4 (2008): 667-681.

Levinson, Arik. "Environmental regulatory competition: A status report and some new evidence." National Tax Journal (2003): 91-106.

List, John A., Azeem M. Shaikh, and Yang Xu. "Multiple hypothesis testing in experimental economics." Experimental Economics 22, no. 4 (2019): 773-793.

Malani, Anup, and Julian Reif. "Interpreting pre-trends as anticipation: Impact on estimated treatment effects from tort reform." Journal of Public Economics 124 (2015): 1-17.

Malo, Nathalie, James A. Hanley, Sonia Cerquozzi, Jerry Pelletier, and Robert Nadon. "Statistical practice in high-throughput screening data analysis." Nature Biotechnology 24, no. 2 (2006): 167-175.

Millimet, Daniel L. "Environmental federalism: a survey of the empirical literature." Case Western Reserve Law Review 64 (2013): 1669.

Morehouse, John, and Edward Rubin. "Do polluters strategically locate near borders? The geography of US power plants and their emissions." Working paper. 2021. 
Neidell, Matthew. "Information, avoidance behavior, and health the effect of ozone on asthma hospitalizations." Journal of Human Resources 44, no. 2 (2009): 450-478.

Oates, Wallace E. A reconsideration of environmental federalism. Resources for the Future Discussion Paper 01-54. 2001.

Oliva, Paulina. "Environmental regulations and corruption: Automobile emissions in Mexico City." Journal of Political Economy 123, no. 3 (2015): 686-724.

Reynaert, Mathias. "Abatement strategies and the cost of environmental regulation: Emission standards on the European car market." The Review of Economic Studies 88, no. 1 (2021): 454-488.

Reynaert, Mathias, and James M. Sallee. "Who Benefits When Firms Game Corrective Policies?." American Economic Journal: Economic Policy 13, no. 1 (2021): 372-412.

Rosenbaum, Paul R. "Overt bias in observational studies." In Observational Studies, pp. 71-104. Springer, New York, NY, 2002.

Schakenbach, John, Robert Vollaro, and Reynaldo Forte. "Fundamentals of successful monitoring, reporting, and verification under a cap-and-trade program." Journal of the Air \& Waste Management Association 56, no. 11 (2006): 1576-1583.

Schwartz, Joel. "Air pollution and blood markers of cardiovascular risk." Environmental Health Perspectives 109, no. suppl 3 (2001): 405-409.

Shapiro, Joseph S., and Reed Walker. Is Air Pollution Regulation Too Stringent? No. w28199. National Bureau of Economic Research, 2020.

Shepard, Donald. "A two-dimensional interpolation function for irregularly-spaced data." In Proceedings of the 1968 23rd ACM national conference, pp. 517-524. 1968.

Shimshack, Jay P. "The economics of environmental monitoring and enforcement: A review." Annual Review of Resource Economics 6 (2014): 339-60.

Simonsohn, Uri, Leif D. Nelson, and Joseph P. Simmons. "P-curve: a key to the file-drawer." Journal of Experimental Psychology: General 143, no. 2 (2014): 534.

Storey, John D. "The positive false discovery rate: a Bayesian interpretation and the q-value." The Annals of Statistics 31, no. 6 (2003): 2013-2035.

Sullivan, Daniel M., and Alan Krupnick. "Using Satellite Data to Fill the Gaps in the US Air Pollution Monitoring Network." Resources for the Future Working Paper (2018): 18-21.

U.S. EPA. Quality Assurance Handbook for Air Pollution Measurement Systems-Volume II-Ambient Air Quality Monitoring Program. Vol. 2. EPA-454/B-13-003 (2013).

Walker, W. Reed. "The transitional costs of sectoral reallocation: Evidence from the clean air act and the workforce." The Quarterly Journal of Economics 128, no. 4 (2013): 1787-1835.

Yang, Lin. "Pollution Monitoring, Strategic Behavior, and Dynamic Representativeness." Working paper (2020).

Young, Alwyn. "Channeling fisher: Randomization tests and the statistical insignificance of seemingly significant experimental results." The Quarterly Journal of Economics 134, no. 2 (2019): 557-598.

Zitzewitz, Eric. "Forensic economics." Journal of Economic Literature 50, no. 3 (2012): 731-69.

Zou, Eric. "Unwatched pollution: The effect of intermittent monitoring on air quality." American Economic Review 111, no. 7 (2021): 2101-26. 
Figure 1. Monitor's Sampling Behavior near Pollution Alerts: Jersey City Firehouse $\mathrm{PM}_{2.5}$ Monitor

Panel A. Event study of monitor's capture rate

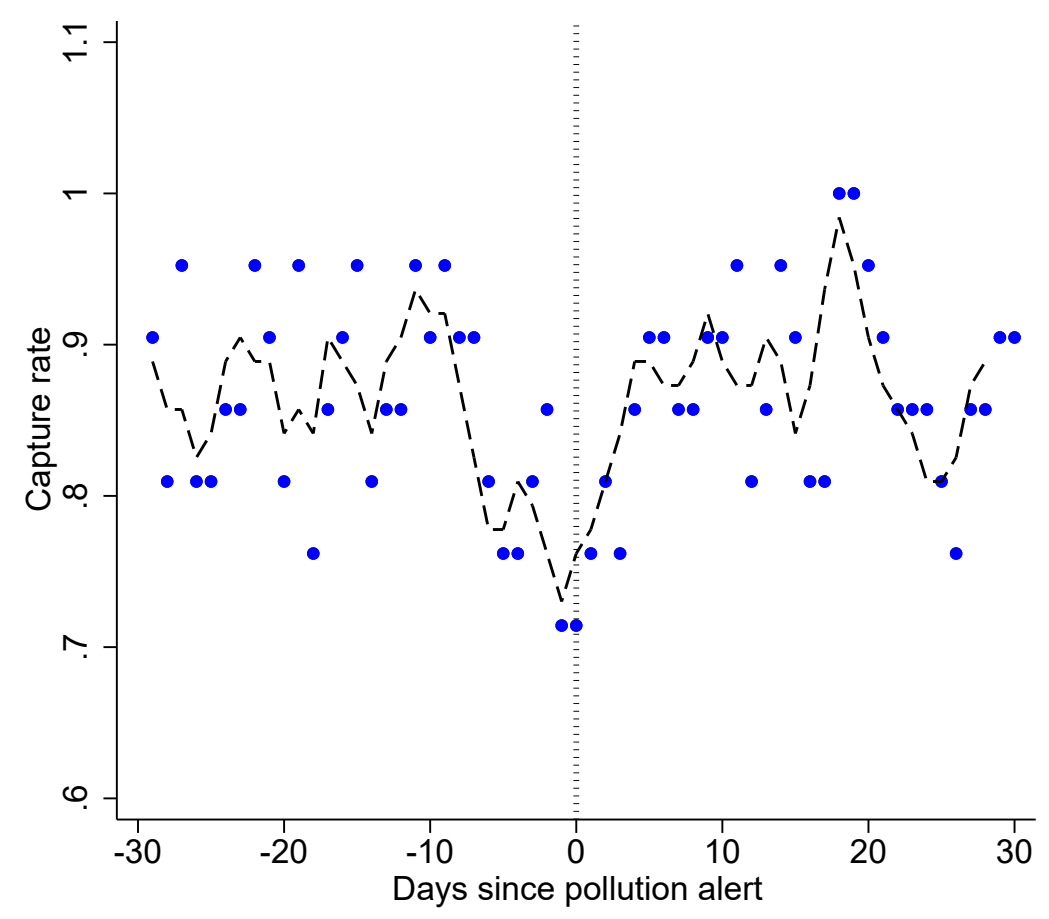

Panel B. Randomized inference with 5,000 placebo scenarios

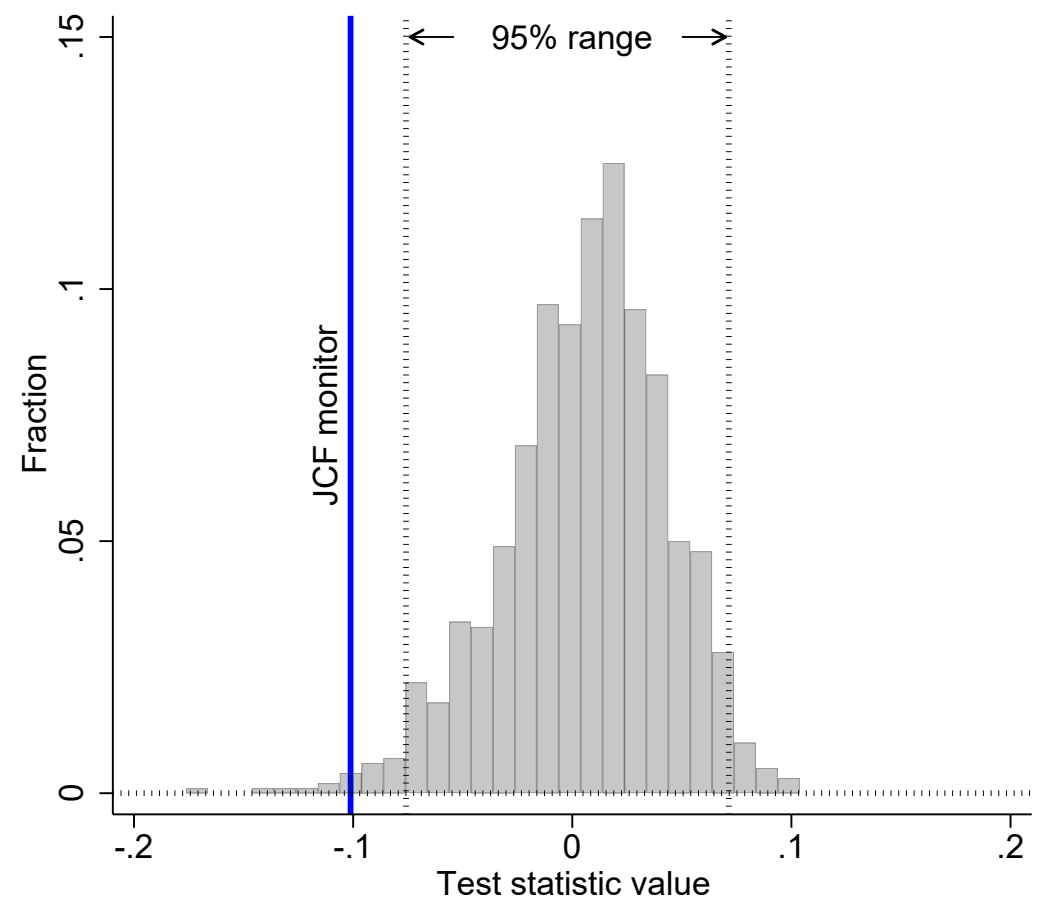

Notes: Panel A plots JCF monitor's average capture rate (i.e., one minus a dummy for missing data) as a function of days since pollution alerts issued by the Jersey City. Number of alerts $=21$. Dashed line represents three-day moving average of point estimates. Panel $B$ plots the distribution of the test statistics derived from 5,000 randomly assigned pollution alerts. Test statistic equals the difference between mean capture rates across event days $[-3,3]$ and mean capture rates across event days $[-30,-10] \cup[10,30]$. Solid vertical line is the observed (i.e., true) test statistic. Dashed vertical lines show $95 \%$ range of the randomized test statistics. 
Figure 2. Distribution of $p$-values, All Monitors

Panel A. Overall

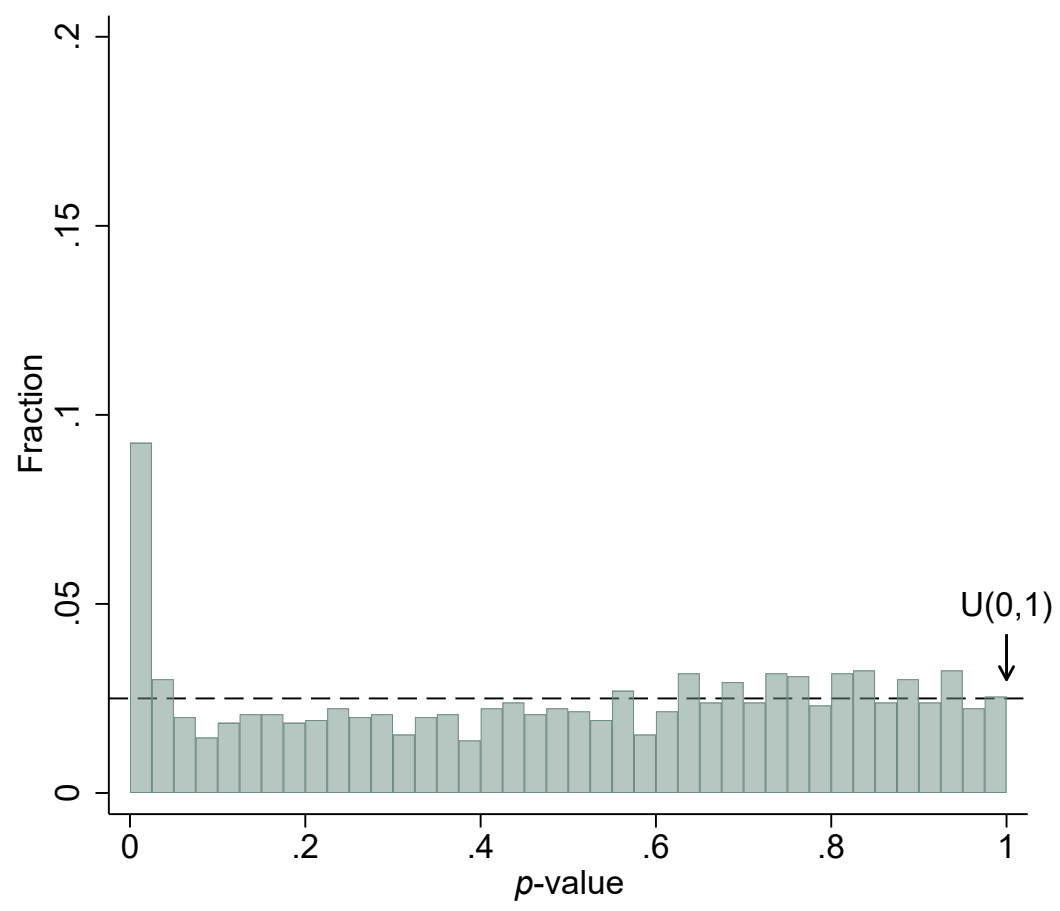

Panel B. By direction of the effect

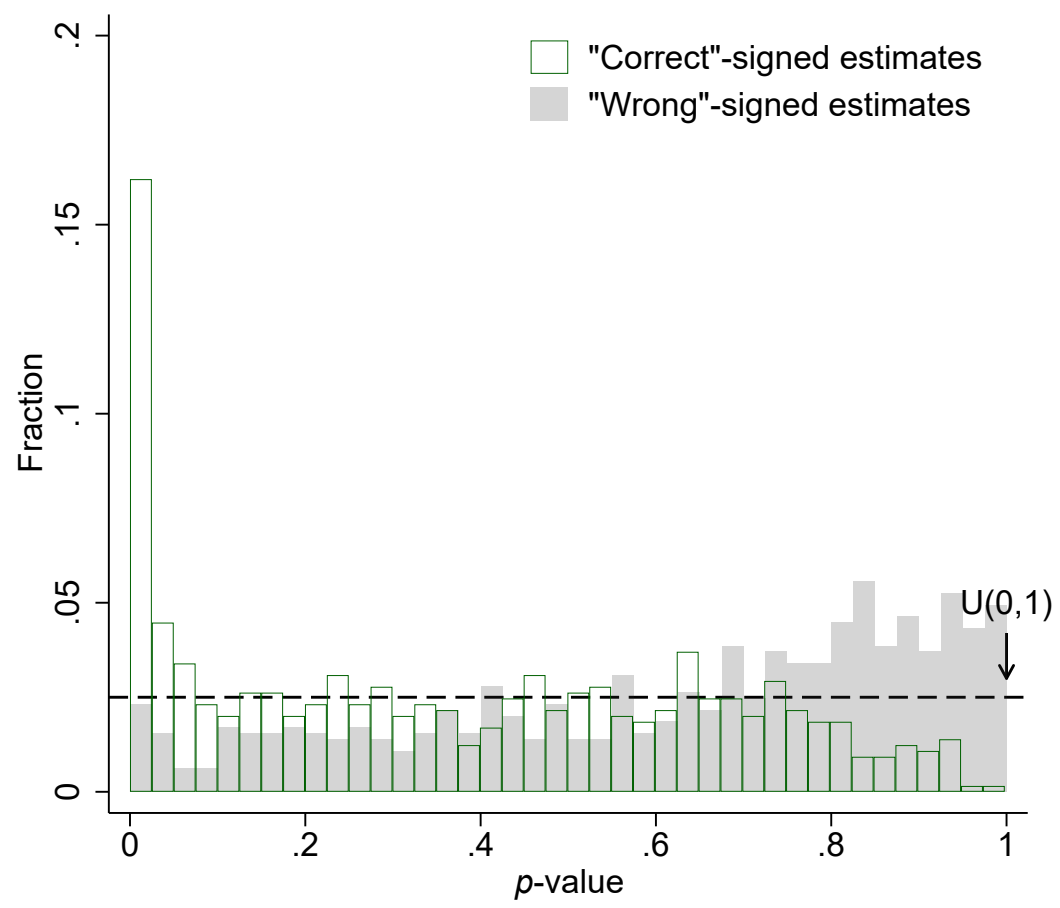

Notes: Panel A shows the distribution of $p$-values for all monitors. Test statistic equals the difference between mean capture rates across event days $[-3,3]$ and mean capture rates across event days $[-30,-10] \cup[10,30]$. Panel $B$ shows the breakdown by the sign of the estimated effect. Hollow bars show $p$-values for negative estimates (i.e., monitoring capture decreases around pollution alerts), and shaded bars show $p$-values for positive estimates (i.e., monitoring capture increases around pollution alerts). Horizontal dashed lines show the uniform distribution. 
Figure 3. Capture Rate for "Interesting" Monitors $(\triangle)$ and Other Monitors $(\bigcirc)$
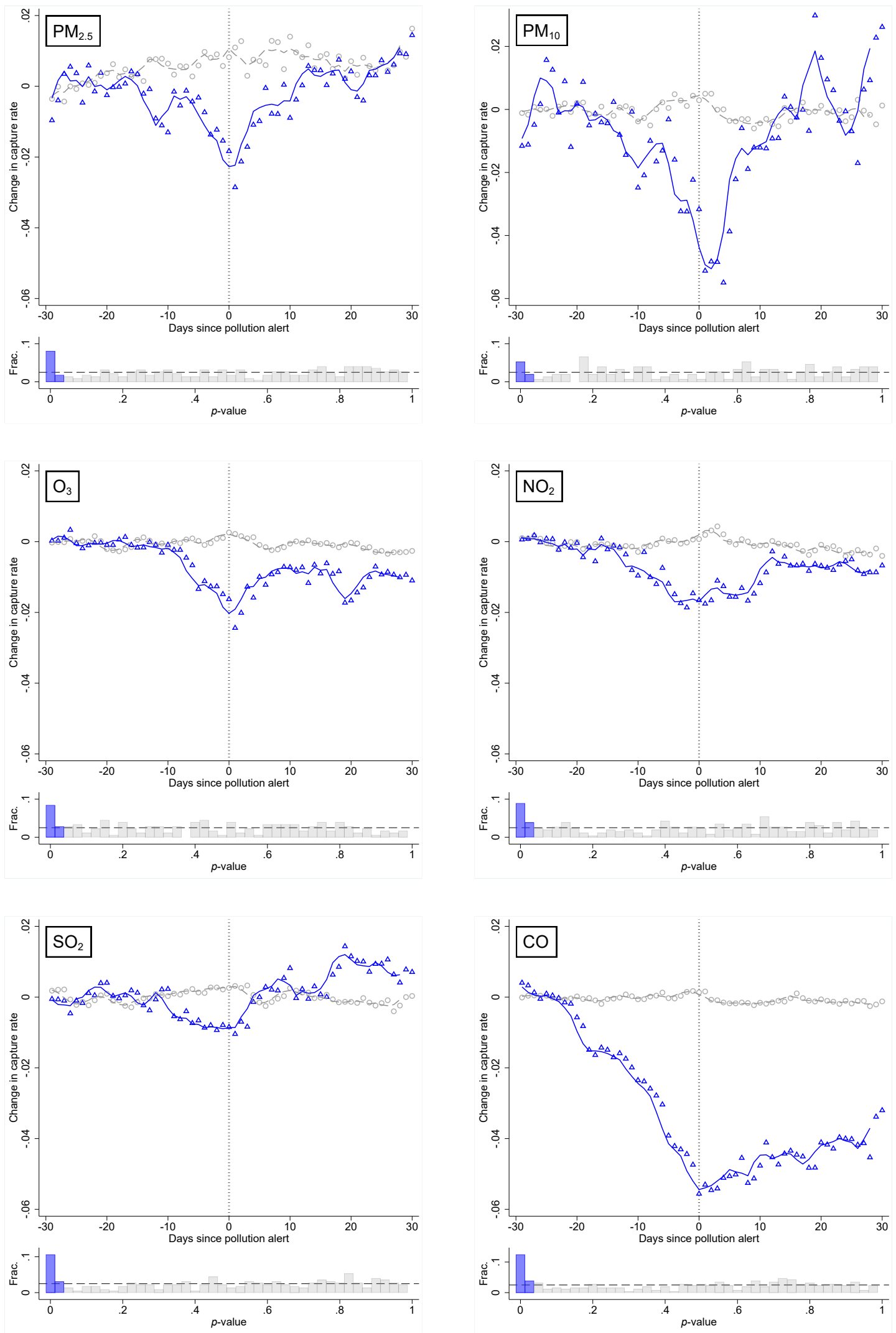

Notes: This graph shows mean monitoring capture rate for "interesting" monitors (those with $p$-value<0.05) and other monitors. Data are demeaned by the average capture rate across the first ten event days. Fitted lines show three-day moving averages of point estimates. Each panel corresponds to one pollutant. Histograms show the distributions of $p$-values for the corresponding pollutant monitors. 
Figure 4. Examples of "Very Interesting" Monitors
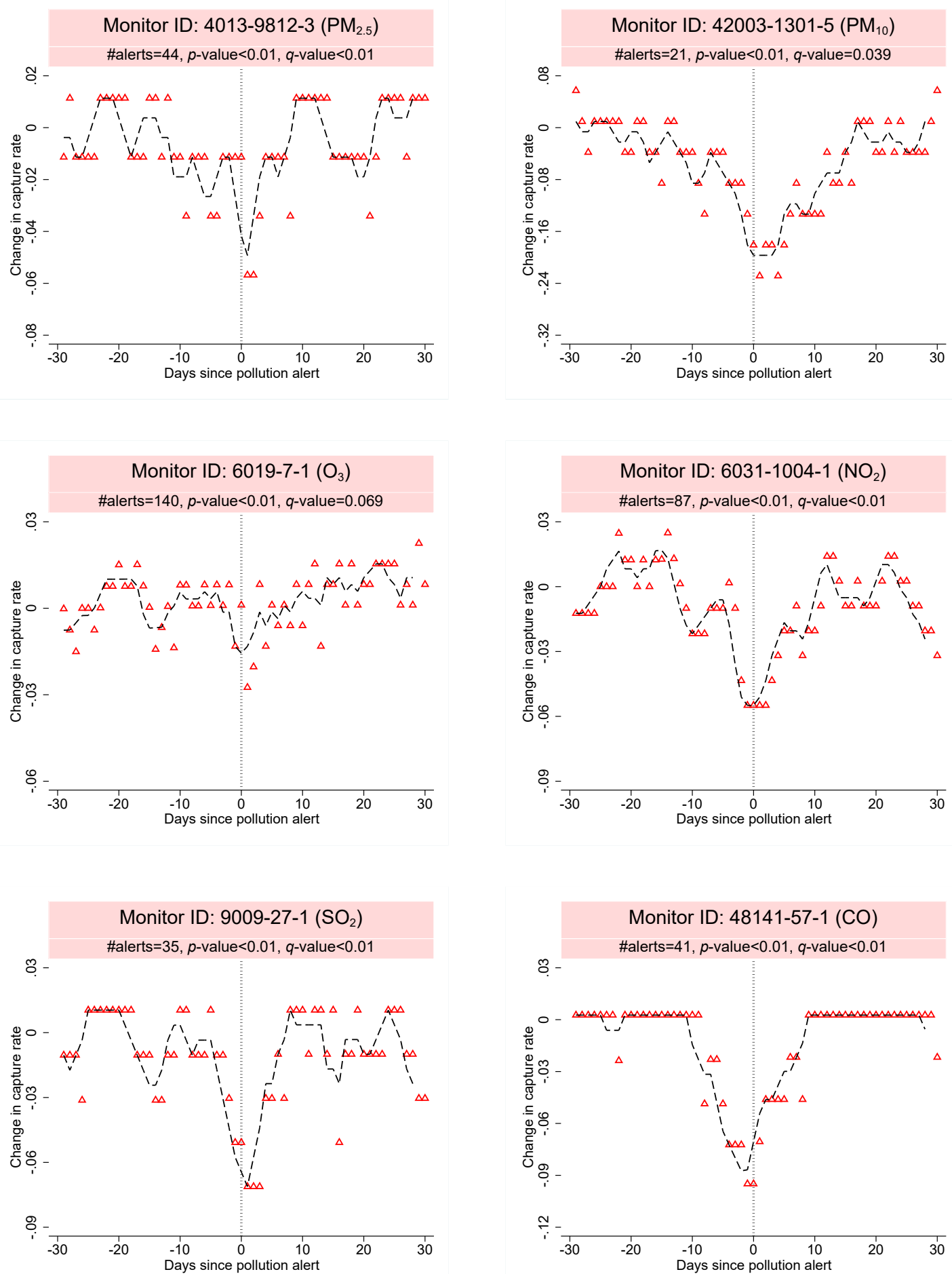

Notes: This graph shows monitor's capture rate for some example "very interesting" monitors (those with $p$-value $<0.05$ and compelling visual pattern). Data are demeaned by the average capture rate across the first ten event days. Fitted lines show three-day moving averages of point estimates. Each panel corresponds to one pollutant. "\#alerts" is total number of city's pollution alerts used in the event study. " $q$-value" is the False Discovery Rate adjusted significance level (Anderson, 2008). 
Figure 5. Study Website: Estimation Results for All Monitors

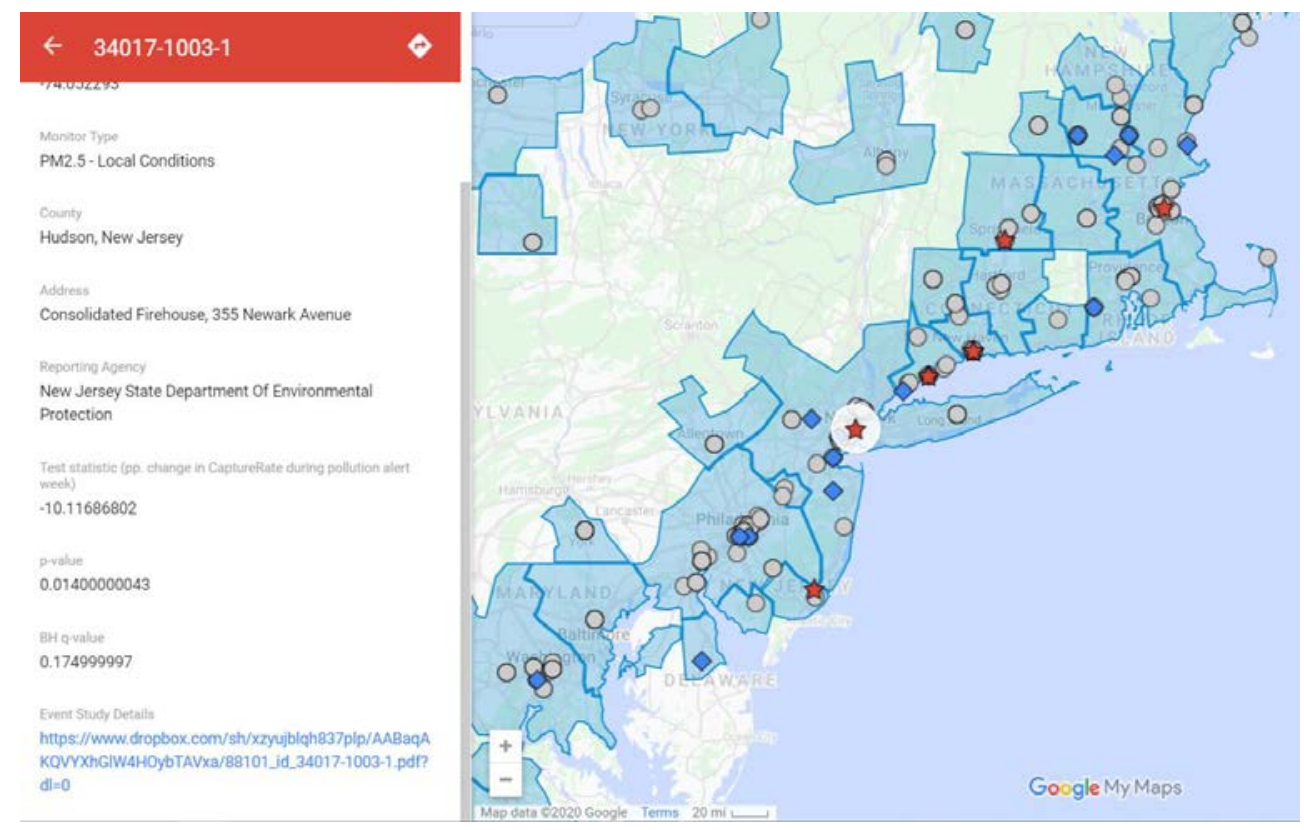

Notes: We store full estimation results at this website. Shaded areas highlight study regions. Click on each monitor to view estimation details. 
Figure 6. Locations of All Tested Monitors $(\bigcirc)$, "Interesting" Monitors $(\triangle)$, and "Very Interesting" Monitors ( $\lesssim$ )
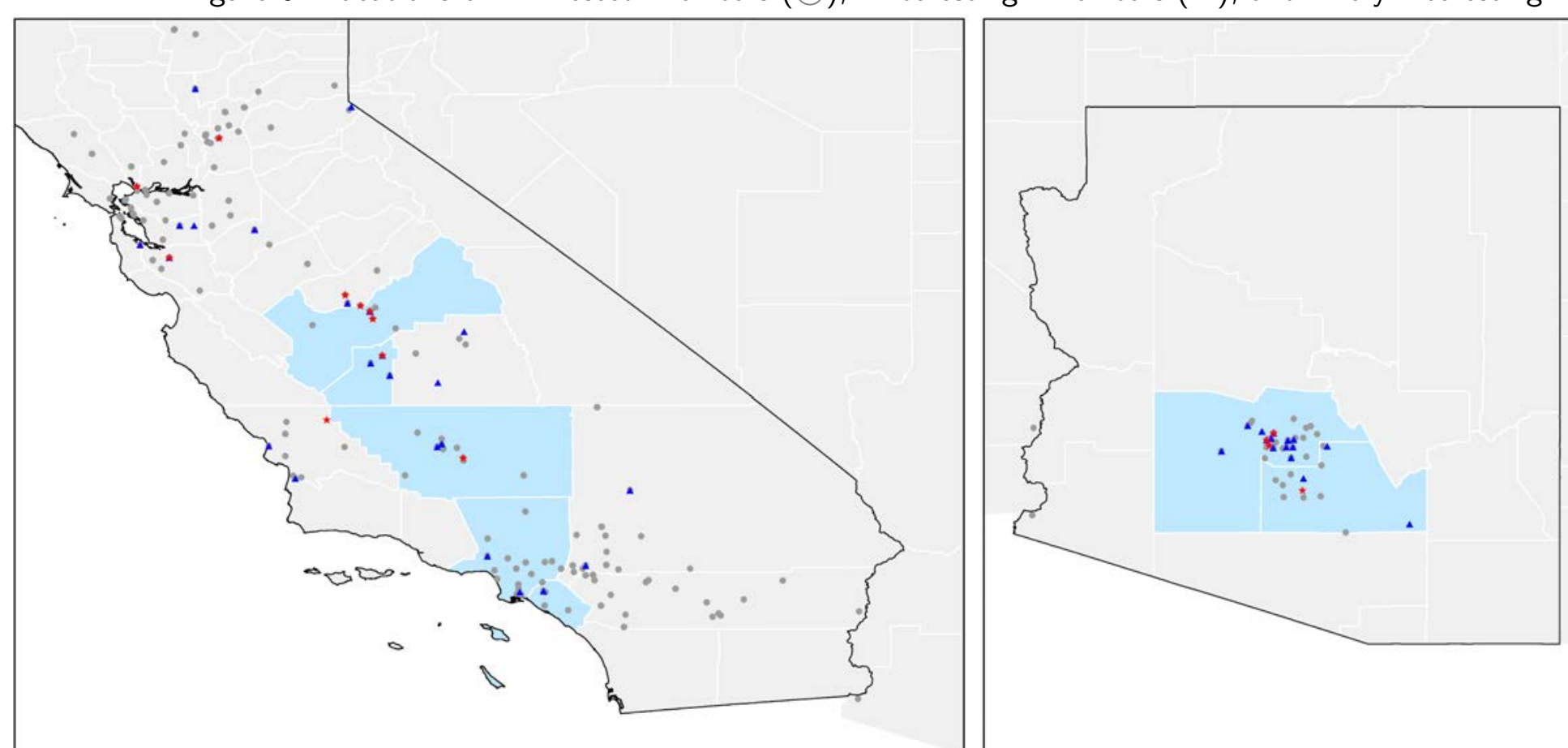

Notes: Blue shades indicate 14 CBSAs that together house $60 \%$ of all "interesting" monitors. Left panel: Bakersfield, CA; Fresno, CA; Hanford-Corcoran, CA; Los Angeles-Long Beach-Anaheim, CA. Right panel: Phoenix-Mesa-Scottsdale, AZ; El Paso, TX. 
Figure 6 (Cont.). Locations of All Tested Monitors ( $\bigcirc)$, "Interesting" Monitors $(\triangle)$, and "Very Interesting" Monitors $(\succsim)$

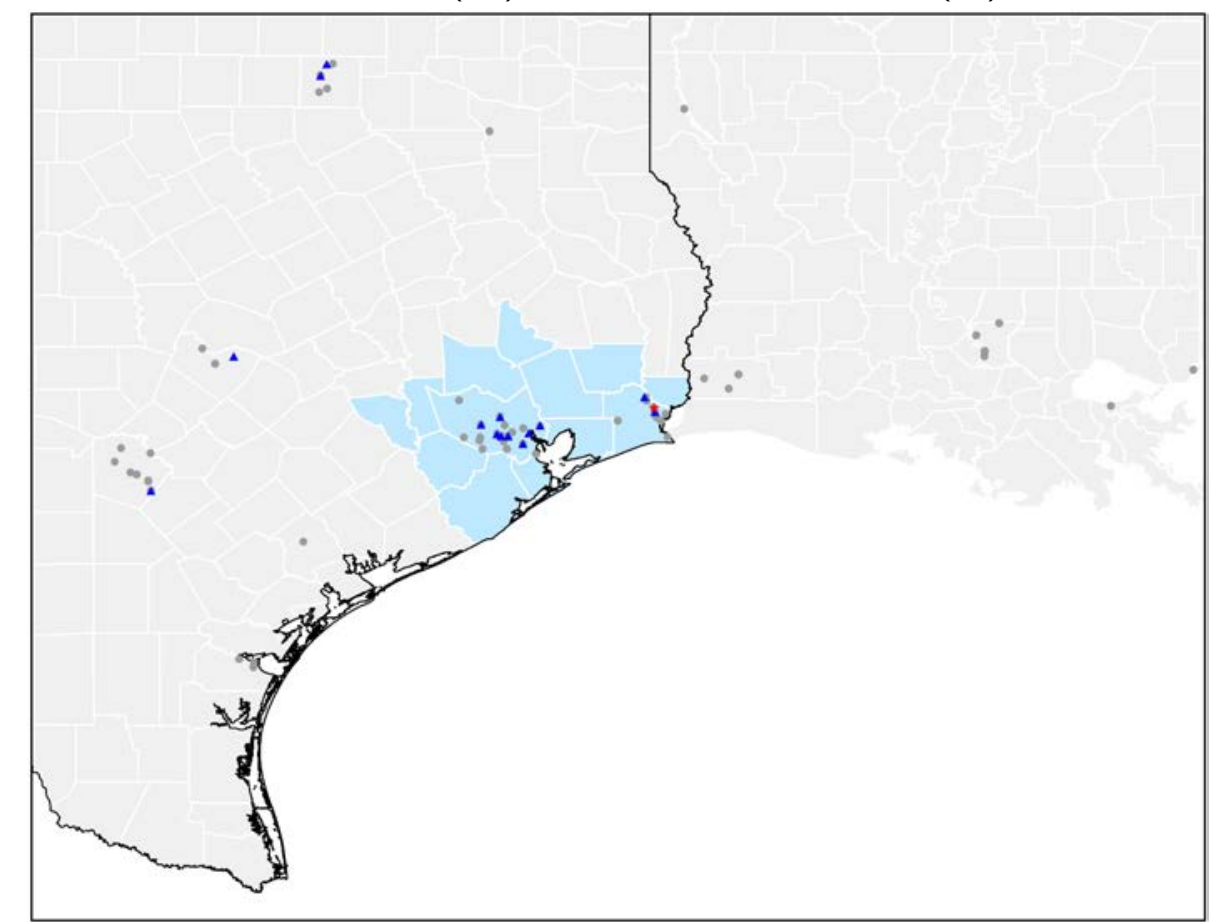

Notes: Blue shades indicate 14 CBSAs that together house $60 \%$ of all "interesting" monitors. Beaumont-Port Arthur, TX; Houston-The Woodlands-Sugar Land, TX. 
Figure 6 (Cont.). Locations of All Tested Monitors $(\bigcirc)$, "Interesting" Monitors $(\triangle)$, and "Very Interesting" Monitors $(ఓ)$
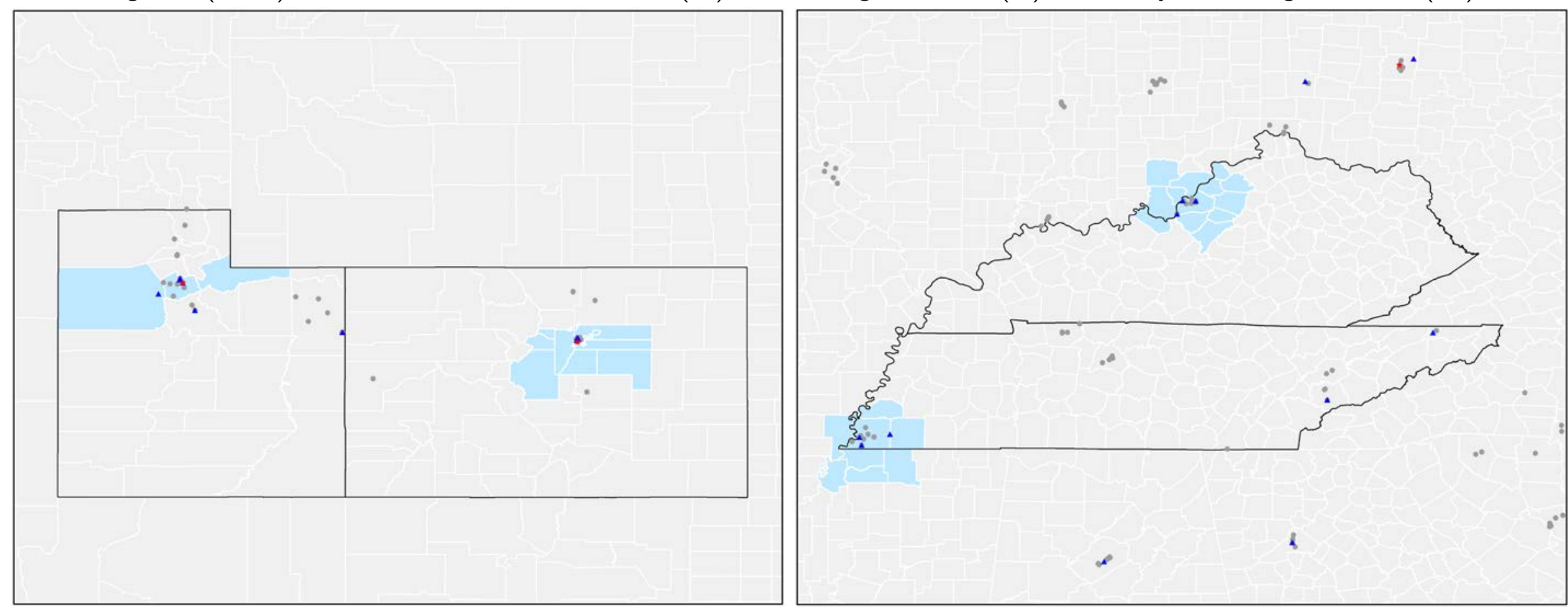

Notes: Blue shades indicate 14 CBSAs that together house $60 \%$ of all "interesting" monitors. Left panel: Salt Lake City, UT; Denver-Aurora-Lakewood, CO. Right panel: Louisville/Jefferson County, KY-IN; Memphis, TN-MS-AR. 
Figure 6 (Cont.). Locations of All Tested Monitors $(\bigcirc)$, "Interesting" Monitors $(\triangle)$, and "Very Interesting" Monitors $(\Sigma)$

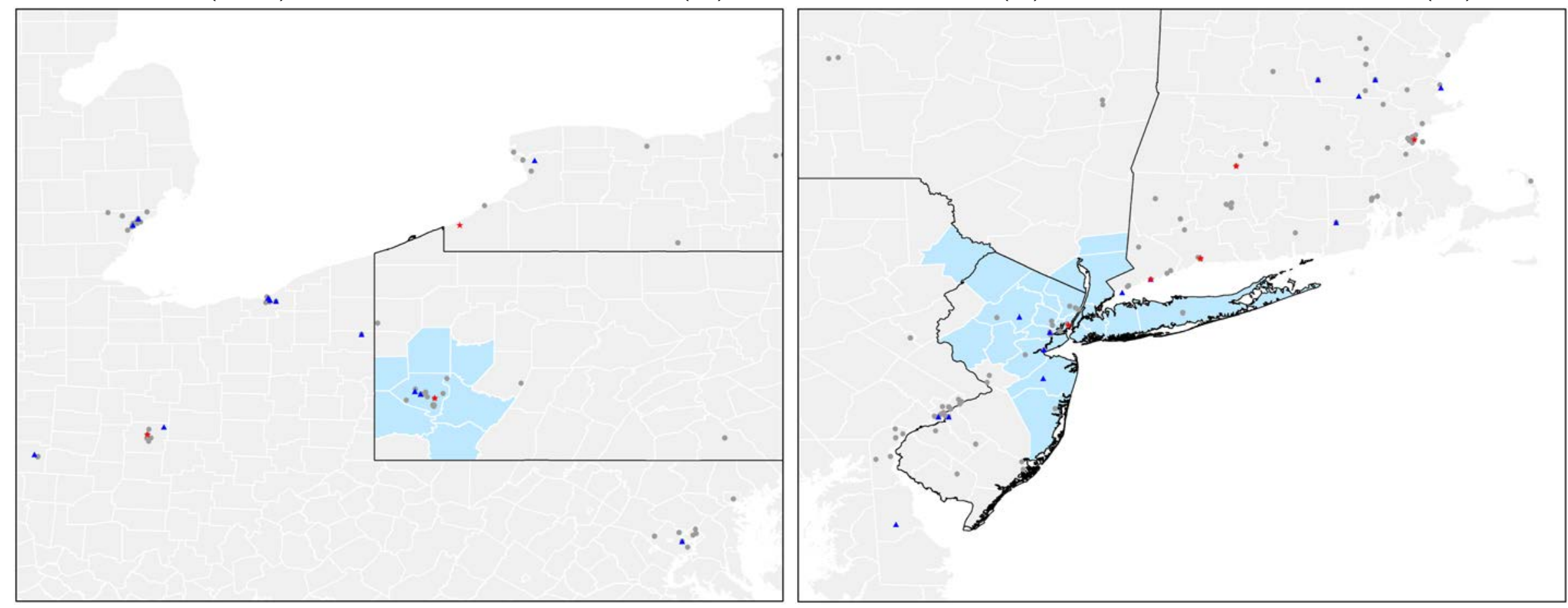

Notes: Left panel: Pittsburgh, PA. Right panel: New York-Newark-Jersey City, NY-NJ-PA. 
Figure 7. Distribution of $p$-values, by Regulatory Status and Pollution Abatement Costs

A. By non-attainment status

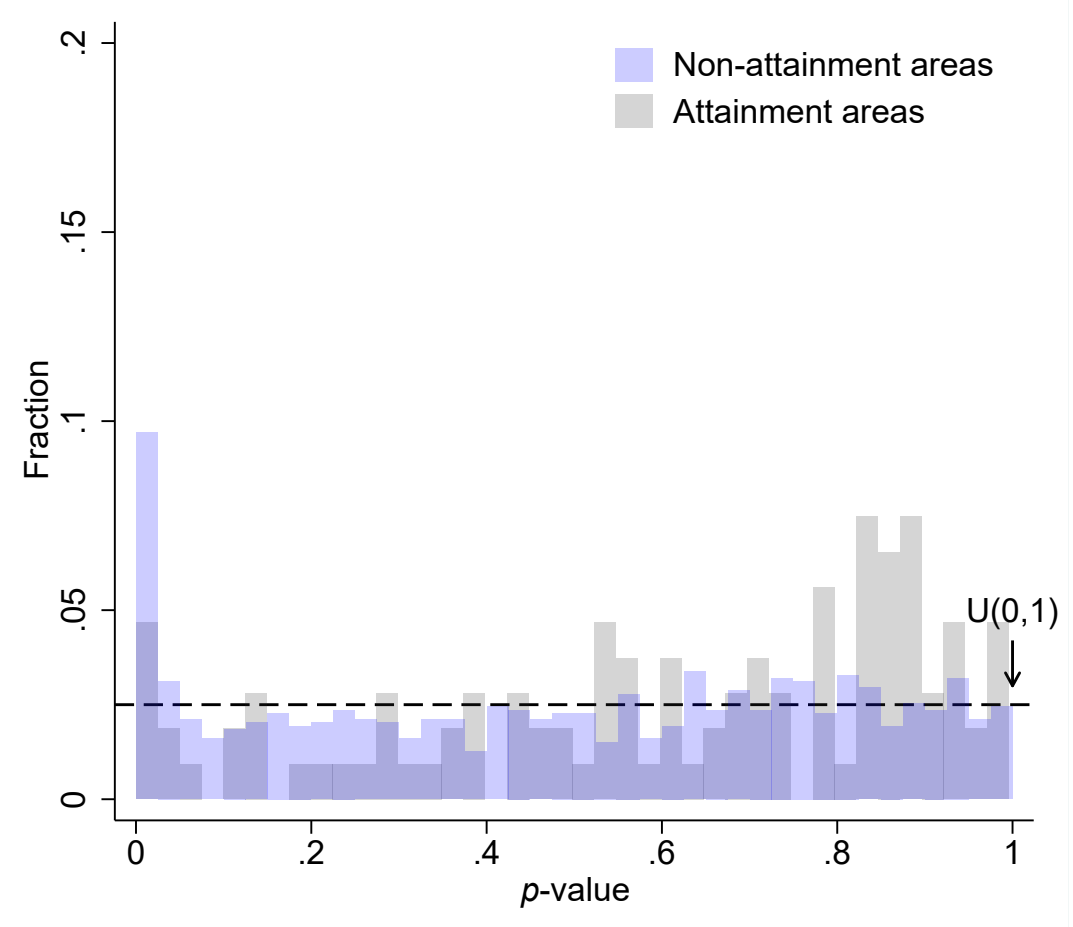

B. Non-attainment areas, by marginal abatement cost (Shapiro and Walker, 2020)
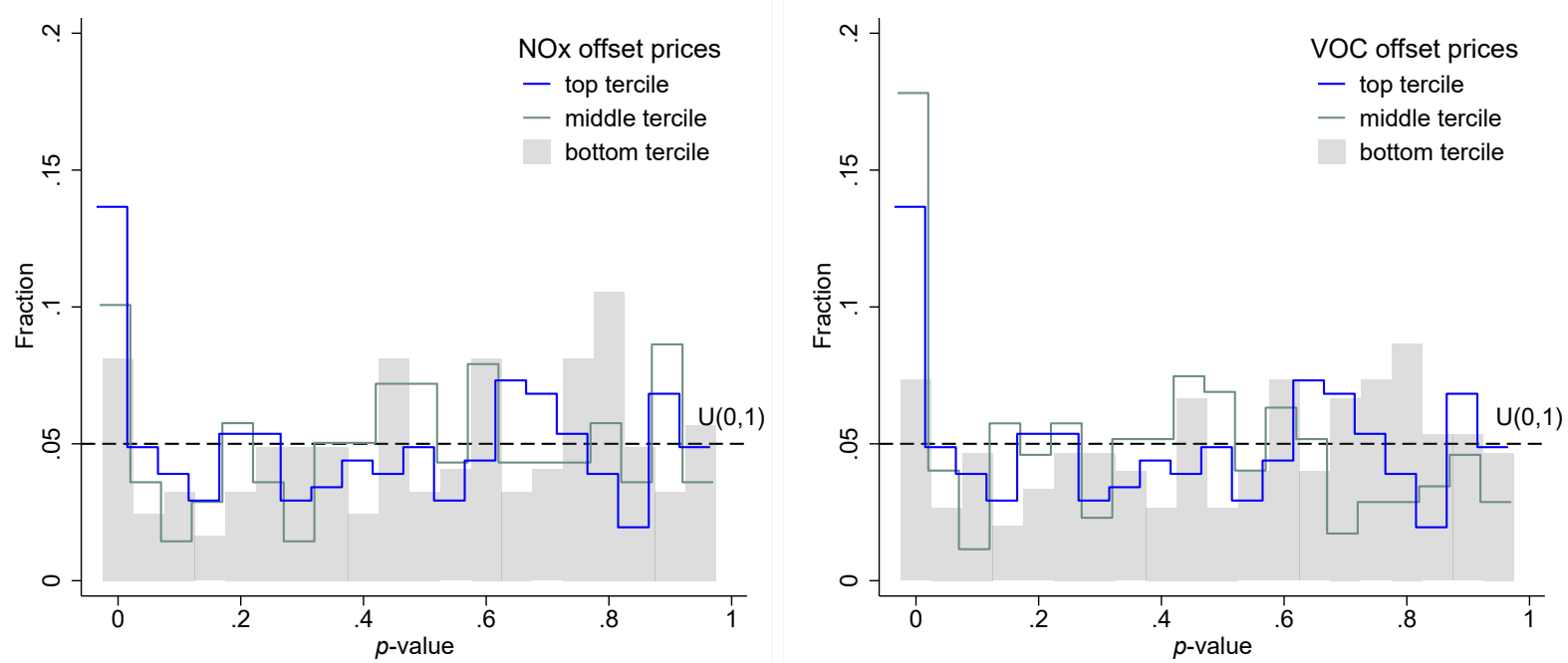

Notes: Panel A shows the breakdown of the distribution of $p$-values by whether the county has ever been in NAAQS non-attainment throughout the study period. Panel B restricts to 582 that located in offset trading markets as documented in Shapiro and Walker (2020), and shows the breakdown of the distribution of $p$-values by deciles of NOx and VOC emission offset prices. In both panels, horizontal dashed lines show the uniform distribution. 
Figure 8. Quarterly Capture Rate vs. Annual $\mathrm{PM}_{2.5}$ Design Value

A. "Interesting" monitors

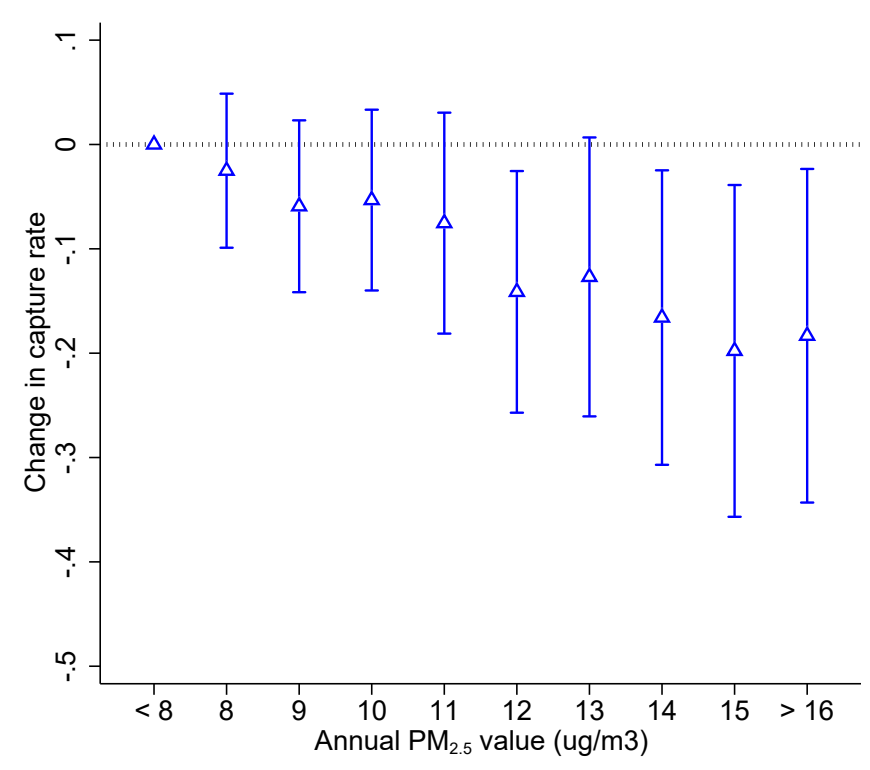

B. Other monitors

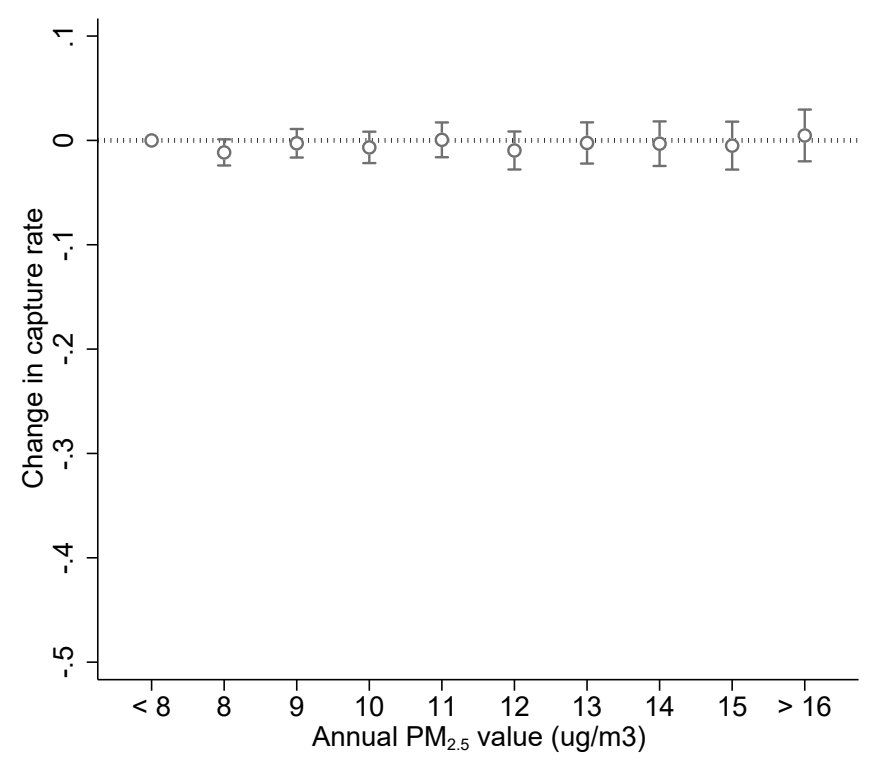

Notes: This figure reports regression of a monitor's quarterly capture rate (valid sampling days divided by required sampling days in a quarter) on $1-\mathrm{ug} / \mathrm{m} 3$ bins of annual mean $\mathrm{PM}_{2.5}$ concentration (i.e., design values for the $\mathrm{PM}_{2.5}$ annual standard). The " $<8$ ug/m3" bin is the omitted category. The regression controls for monitor fixed effects and year fixed effects. The regression is run separately for "interesting" monitors (panel A, number of observations $=300$ ) and other monitors (panel B, number of observations $=7,688$ ). 
Figure 9. Distributions of Observed and Imputed $\mathrm{PM}_{2.5}$ Concentration

A. Imputation method: inverse distance weighting (IDW)
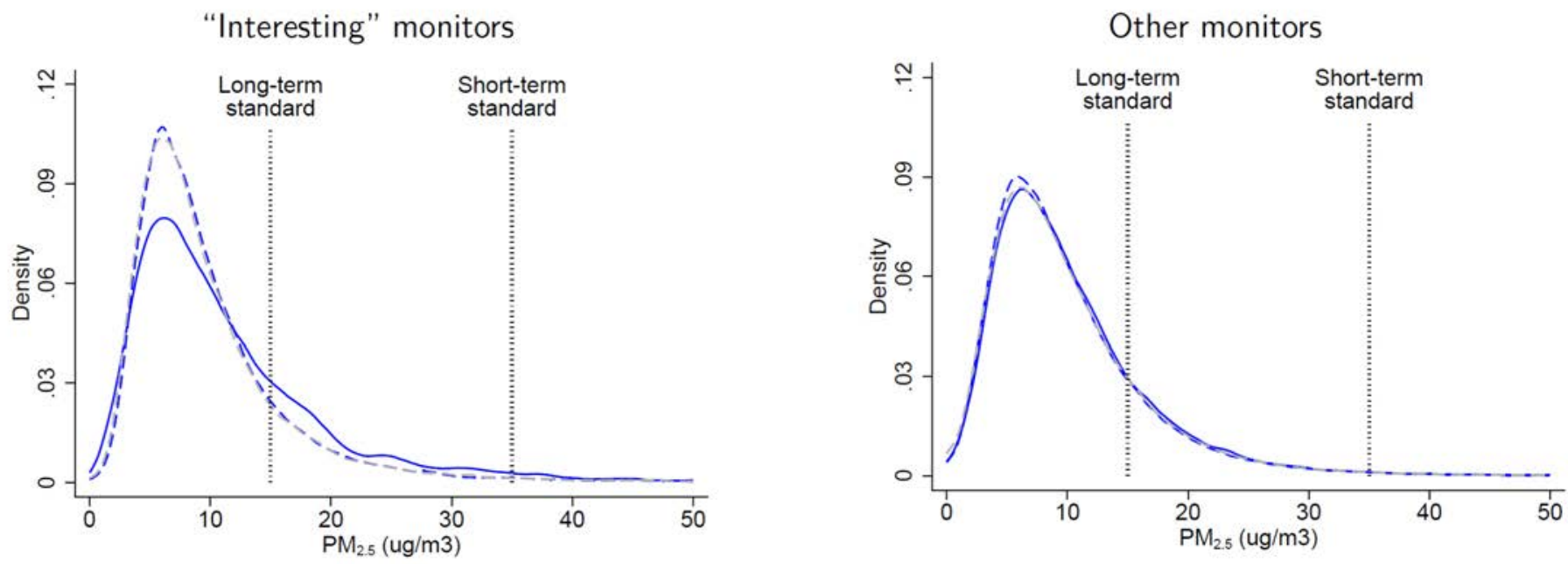

----- Observed value, when not missing monitoring
---- Predicted value, when not missing monitoring
- Predicted value, when missing monitoring

B. Imputation method: atmospheric modeling (Di et al., 2019)

"Interesting" monitors

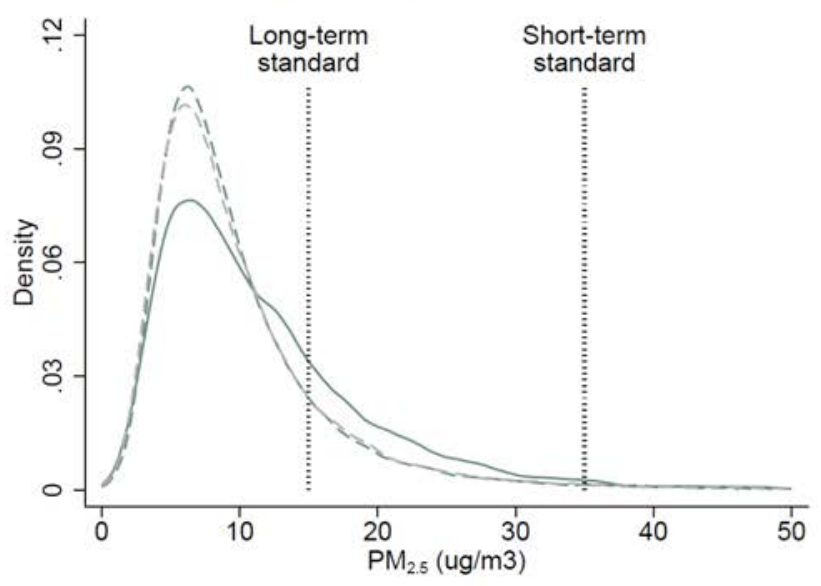

Other monitors

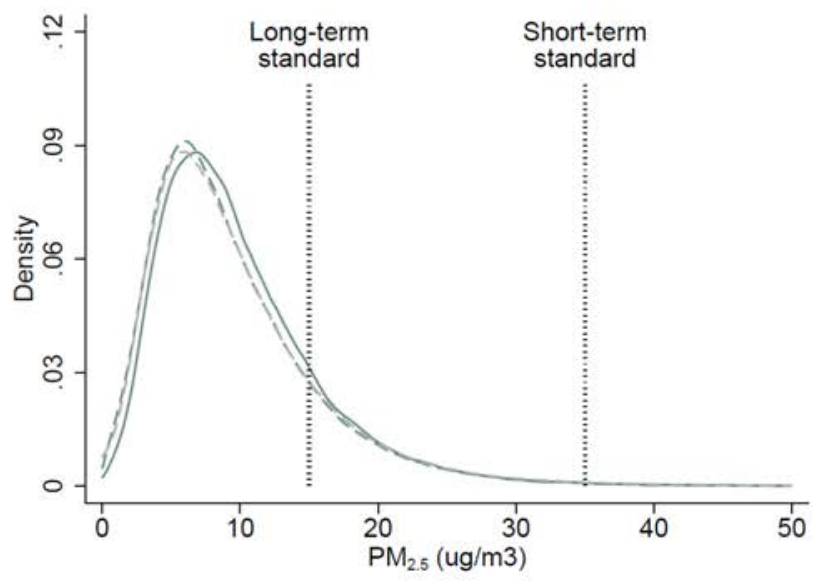

---- Observed value, when not missing monitoring
---- Predicted value, when not missing monitoring
Predicted value, when missing monitoring

Notes: Underlying data are monitor-daily level average $\mathrm{PM}_{2.5}$ concentration. "Observed value" is concentration recorded on the monitorday. In panel A, "Predicted value" is inverse distance-weighted concentration from all other operative PM $\mathrm{PM}_{2.5} \mathrm{monitors}$ within a 20 -mile radius. In panel B, "Predicted value" is from $1 \mathrm{~km} \times 1 \mathrm{~km}$ grid-daily prediction of $\mathrm{PM}_{2.5}$ from atmospheric ensemble-based modeling ( Di et al., 2019) which incorporates satellite observations. "Long-term standard" marks the 15 ug/m3 annual NAAQS standard. "Shortterm standard" marks the $35 \mathrm{ug} / \mathrm{m} 3$ 24-hr NAAQS standard. 
Table 1. List of CBSAs, Ranked by Cases of "Interesting" Monitors $(\triangle)$

\begin{tabular}{|c|c|c|c|c|}
\hline CBSA & \#alerts & 0 & $\triangle$ & 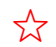 \\
\hline Phoenix-Mesa-Scottsdale, AZ & 51 & 86 & 23 & 4 \\
\hline Houston-The Woodlands-Sugar Land, TX & 199 & 49 & 12 & 0 \\
\hline Denver-Aurora-Lakewood, CO & 30 & 21 & 6 & 1 \\
\hline Fresno, CA & 83 & 32 & 6 & 4 \\
\hline Salt Lake City, UT & 66 & 21 & 5 & 1 \\
\hline Hanford-Corcoran, CA & 66 & 9 & 5 & 1 \\
\hline New York-Newark-Jersey City, NY-NJ-PA & 30 & 33 & 5 & 1 \\
\hline Memphis, TN-MS-AR & 18 & 13 & 4 & 0 \\
\hline El Paso, TX & 44 & 30 & 4 & 1 \\
\hline Los Angeles-Long Beach-Anaheim, CA & 22 & 72 & 4 & 0 \\
\hline Beaumont-Port Arthur, TX & 45 & 19 & 4 & 1 \\
\hline Pittsburgh, PA & 64 & 29 & 4 & 1 \\
\hline Louisville/Jefferson County, KY-IN & 27 & 18 & 4 & 0 \\
\hline Bakersfield, CA & 71 & 29 & 4 & 1 \\
\hline San Luis Obispo-Paso Robles-Arroyo Grande, CA & 17 & 16 & 3 & 1 \\
\hline San Jose-Sunnyvale-Santa Clara, CA & 18 & 11 & 3 & 1 \\
\hline San Francisco-Oakland-Hayward, CA & 32 & 57 & 3 & 0 \\
\hline Cleveland-Elyria, $\mathrm{OH}$ & 50 & 15 & 3 & 0 \\
\hline Boston-Cambridge-Newton, MA-NH & 19 & 27 & 3 & 1 \\
\hline Bridgeport-Stamford-Norwalk, CT & 40 & 10 & 3 & 1 \\
\hline Knoxville, TN & 50 & 7 & 2 & 0 \\
\hline Dallas-Fort Worth-Arlington, TX & 78 & 11 & 2 & 0 \\
\hline Modesto, CA & 82 & 10 & 2 & 0 \\
\hline Grand Rapids-Wyoming, MI & 10 & 5 & 2 & 0 \\
\hline Madera, CA & 45 & 9 & 2 & 1 \\
\hline Visalia-Porterville, CA & 91 & 7 & 2 & 0 \\
\hline Detroit-Warren-Dearborn, MI & 15 & 31 & 2 & 0 \\
\hline Philadelphia-Camden-Wilmington, PA-NJ-DE-MD & 24 & 51 & 2 & 0 \\
\hline Riverside-San Bernardino-Ontario, CA & 21 & 77 & 2 & 0 \\
\hline New Haven-Milford, CT & 35 & 9 & 2 & 2 \\
\hline Austin-Round Rock, TX & 28 & 6 & 2 & 0 \\
\hline Raleigh, NC & 22 & 7 & 2 & 0 \\
\hline Manchester-Nashua, NH & 11 & 9 & 2 & 0 \\
\hline Vernal, UT & 14 & 9 & 2 & 0 \\
\hline Sacramento-Roseville-Arden-Arcade, CA & 22 & 50 & 2 & 1 \\
\hline Columbus, $\mathrm{OH}$ & 28 & 8 & 2 & 1 \\
\hline Atlanta-Sandy Springs-Roswell, GA & 60 & 8 & 2 & 0 \\
\hline Kingsport-Bristol-Bristol, TN-VA & 35 & 4 & 1 & 0 \\
\hline Chicago-Naperville-Elgin, IL-IN-WI & 25 & 29 & 1 & 0 \\
\hline Lawton, OK & 3 & 1 & 1 & 0 \\
\hline Buffalo-Cheektowaga-Niagara Falls, NY & 25 & 10 & 1 & 0 \\
\hline Dayton, $\mathrm{OH}$ & 37 & 3 & 1 & 0 \\
\hline Birmingham-Hoover, AL & 63 & 14 & 1 & 0 \\
\hline San Antonio-New Braunfels, TX & 13 & 13 & 1 & 0 \\
\hline Washington-Arlington-Alexandria, DC-VA-MD-WV & 3 & 19 & 1 & 0 \\
\hline Springfield, MA & 36 & 7 & 1 & 1 \\
\hline Jamestown-Dunkirk-Fredonia, NY & 33 & 2 & 1 & 1 \\
\hline Providence-Warwick, RI-MA & 9 & 13 & 1 & 0 \\
\hline Dover, DE & 14 & 1 & 1 & 0 \\
\hline Yuba City, CA & 4 & 3 & 1 & 0 \\
\hline Gulfport-Biloxi-Pascagoula, MS & 4 & 5 & 1 & 0 \\
\hline Vallejo-Fairfield, CA & 51 & 11 & 1 & 1 \\
\hline Provo-Orem, UT & 101 & 9 & 1 & 1 \\
\hline Youngstown-Warren-Boardman, OH-PA & 6 & 4 & 1 & 0 \\
\hline
\end{tabular}

Notes: \#alerts $=$ number of alerts issued during the study period. $\bigcirc=$ number of tested monitors; $\Delta=$ number of "interesting" monitors; $\hat{s}=$ number of "very interesting" monitors. 
Table 2. Correlates of "Interesting" Monitors

(1) (2) (3) (4) (5)

5)

5)

(6)

(7)

(8)

Dep. var.:

$1(p$-value $\leq 0.05)$

$1(q$-value $\leq 0.05)$

Non-attainment

$0.066 * *$

(0.030)

$0.039 *$

(0.021)

Non-attainment $\times 1$ ("wrong" sign)

$\begin{array}{ccc}-0.014 & 0.011 & -0.001 \\ (0.033) & (0.034) & (0.041)\end{array}$

Non-attainment $\times 1$ ("correct" sign)

$0.203 * * * \quad 0.220 * * * \quad 0.223 * * *$

$\begin{array}{lll}(0.055) & (0.055) \quad(0.061)\end{array}$

$-0.022$

$(0.027)$

(0.061)

$-0.023$

(0.027)

$1(q$-value $\leq 0.05)$

Above median Democrats

0.007

$(0.017)$

$\begin{array}{ccc}-0.002 & 0.012 & 0.022 \\ (0.024) & (0.024) & (0.030)\end{array}$

$0.111^{* * *} \quad 0.124 * * * \quad 0.129 * * *$

$\begin{array}{lll}(0.039) & (0.039) \quad(0.044)\end{array}$

$-0.014$

(0.019)

Above median LCV score

$0.035^{*}$

$-0.021$

(0.019)

Above median government size

$(0.018)$

$-0.001$

(0.012)

0.008

(0.013)

State fixed effects

Mean dep. var.

0.117

0.117

0.117

1,359

1,359

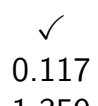

0.117

1,359

0.052

1,359

0.052

1,359

0.052

1,359

$\checkmark$

Observations

Notes: Each column is a separate regression. Underlying data is a cross-section of monitors matched to parenting county's characteristics. Dependent variable is an indicator for whether the monitor's $p$-value is less than 0.05 (columns $1-4$ ), or an indicator for whether the monitor's FDR-adjusted significance $q$-value is less than 0.05 (columns 5-8) where the family of tests is all 1,359 monitors. "Nonattainment" is an indicator for whether the county has ever been in NAAQS non-attainment throughout the study period. "1("correct" sign)" indicates a negative effect sign, i.e., capture rate drops near pollution alerts. "Above median"'s indicate the county has an above-median level of share of Democrats affiliation (2006 Gallup Poll), League of Conservation Voters score, share of government-sector employees (Bureau of Economic Analysis, NAICS=92), and per-capita number of federal convictions among state and local public officials (Glaeser and Saks, 2006). *: $p<0.10 ; * *: p<0.05 ; * * *: p<0.01$. 


\section{Appendix A. Proof of Propositions}

\section{Setup}

We consider a setting in which a regulator sets a standard for a regulated entity, and the regulated entity generates data to document its level of compliance with the regulator's standard. The regulated entity-the monitor henceforth-has private information (the local record of compliance) that the regulator desires (i.e., wants the local monitor to report truthfully). The regulator then penalizes the regulated entity based upon its lack of compliance to the standard - and potentially for missing observations. Finally, we allow for the possibility that the monitor incurs a cost for each observation it produces. Consider the following timeline:

1. Regulator sets standard: The regulator sets a standard for the regulated activity.

2. Monitor observes state: The monitor observes the state of the world. The regulator and the monitor know that there are $N$ observations in the period. Let $n_{v} \in(0, N)$ denote observations that violate the standard. There are thus $n_{c}=N-n_{v}$ observations that comply with the standard.

3. Monitor generates and reports data: The monitor chooses which observations to record and reports them to the regulator. The monitor generates data for $r_{v}\left(\leq n_{v}\right)$ violating observations and $r_{c}\left(\leq n_{c}\right)$ compliant observations. The monitor reports $\left\{r_{v}, r_{c}\right\}$ to the regulator. Reported records each cost the monitor $c$, i.e., in the absence of penalties, the monitor incurs a total cost of $c \times\left(r_{v}+r_{c}\right)$.

4. Regulator penalizes monitor: The regulator imposes penalties. The penalty for $r_{v}$ reported violations is $p_{v}\left(r_{v}\right)$. The penalty for $N-r_{v}-r_{c}$ missing observations is $p_{m}\left(N-r_{v}-r_{c}\right)$. In many cases, $p_{v}$ and $p_{m}$ are both likely convex for non-negative $r_{v}$ and $r_{c}$ (i.e., $p_{v}^{\prime \prime}>0$ and $p_{m}^{\prime \prime}>0$ ): some degree of violations or missingness are tolerated (i.e., $p_{v}^{\prime}(0)$ and $p_{m}^{\prime}(0)$ are relatively small), but as the violations or missingness increase, so does the marginal penalty. Here we assume the two penalties are additively separable.

\section{Minimizing costs}

Backsolving, the monitor chooses $r_{v}$ and $r_{c}$ (assumed continuous) that minimize the sum of its penalties $p_{v}\left(r_{v}\right)+p_{m}\left(N-r_{v}-r_{c}\right)$ and costs $c \times\left(r_{v}+r_{c}\right)$. Thus, the monitor's problem is

$$
\min _{r_{v}, r_{c}} C=p_{v}\left(r_{v}\right)+p_{m}\left(N-r_{v}-r_{c}\right)+c \times\left(r_{v}+r_{c}\right) \quad \text { s.t. } \quad 0 \leq r_{v} \leq n_{v} \text { and } 0 \leq r_{c} \leq n_{c}
$$

Flipping equation A to a constrained maximization problem,

$$
\max _{r_{v}, r_{c}} \mathscr{L}=-p_{v}\left(r_{v}\right)-p_{m}\left(N-r_{v}-r_{c}\right)-c \times\left(r_{v}+r_{c}\right)+\lambda_{1}\left(n_{v}-r_{v}\right)+\lambda_{2}\left(n_{c}-r_{c}\right)+\lambda_{3} r_{v}+\lambda_{4} r_{c}
$$




\section{First-order conditions}

The Karesh-Kuhn-Tucker (KKT) conditions for equation B imply ten first-order conditions:

$$
\begin{aligned}
\frac{\partial \mathscr{L}}{\partial r_{v}} & =-p_{v}^{\prime}+p_{m}^{\prime}-c+\lambda_{1}+\lambda_{3}=0 \\
\frac{\partial \mathscr{L}}{\partial r_{c}} & =p_{m}^{\prime}-c+\lambda_{2}+\lambda_{4}=0 \\
\frac{\partial \mathscr{L}}{\partial \lambda_{1}} & =n_{v}-r_{v} \geq 0 \\
\frac{\partial \mathscr{L}}{\partial \lambda_{2}} & =n_{c}-r_{c} \geq 0 \\
\frac{\partial \mathscr{L}}{\partial \lambda_{3}} & =r_{v} \geq 0 \\
\frac{\partial \mathscr{L}}{\partial \lambda_{4}} & =r_{c} \geq 0 \\
\frac{\partial \mathscr{L}}{\partial \lambda_{1}} \lambda_{1} & =\left(n_{v}-r_{v}\right) \lambda_{1}=0 \\
\frac{\partial \mathscr{L}}{\partial \lambda_{2}} \lambda_{2} & =\left(n_{c}-r_{c}\right) \lambda_{2}=0 \\
\frac{\partial \mathscr{L}}{\partial \lambda_{3}} \lambda_{3} & =\left(r_{v}\right) \lambda_{3}=0 \\
\frac{\partial \mathscr{L}}{\partial \lambda_{4}} \lambda_{4} & =\left(r_{c}\right) \lambda_{4}=0
\end{aligned}
$$

To consider the the feasible equilibria implied by this model, we examine four general cases:

1. No reporting: $r_{v}=0$ and $r_{c}=0$

2. No reporting of violations: $r_{c}>r_{v}=0$

3. No reporting of compliance: $r_{v}>r_{c}=0$

4. Non-zero reporting of violations and compliance: $r_{v}>0$ and $r_{c}>0$

Note that complete, honest reporting is a special subcase of case 4.

Case 1: No reporting $\left(r_{v}=0\right.$ and $\left.r_{c}=0\right)$

In this scenario, KKT first-order conditions $\mathscr{L}_{3}-\mathscr{L}_{6}, \mathscr{L}_{9}$, and $\mathscr{L}_{10}$ are automatically satisfied. Because $\mathscr{L}_{3}$ and $\mathscr{L}_{4}$ are satisfied with inequalities, satisfying $\mathscr{L}_{7}$ and $\mathscr{L}_{8}$ requires $\lambda_{1}=0$ and $\lambda_{2}=0$ (respectively). Thus, $\mathscr{L}_{1}$ becomes

$$
\begin{array}{r}
-p_{v}^{\prime}(0)+p_{m}^{\prime}(N)-c+\lambda_{3}=0 \\
\Longrightarrow \lambda_{3}=p_{v}^{\prime}(0)-p_{m}^{\prime}(N)+c \geq 0 \\
\Longrightarrow p_{v}^{\prime}(0)+c \geq p_{m}^{\prime}(N)
\end{array}
$$


Similarly, $\mathscr{L}_{2}$ becomes

$$
\begin{array}{r}
p_{m}(N)^{\prime}-c+\lambda_{4}=0 \\
\Longrightarrow \lambda_{4}=-p_{m}^{\prime}(N)+c \geq 0 \\
\Longrightarrow c \geq p_{m}^{\prime}(N)
\end{array}
$$

Therefore, in order for $\lambda_{4} \geq 0$ and for this case to be feasible, it must be true that $c \geq p_{m}^{\prime}(N)$. In other words, when the marginal costs of monitoring/reporting are greater than the marginal penalty for missingness when all observations are missing, then the monitor will not report any observations-neither compliance, nor violations. (The costs or reporting exceed the fines of not reporting-even at extreme non-reporting.) This case is really only feasible when monitoring costs are very large or when regulators impose essentially no penalties for missing observations.

Case 2: No reporting of violations $\left(r_{c}>r_{v}=0\right)$

Here, $r_{v}=0$ satisfies $\mathscr{L}_{3}$ with inequality, so $\lambda_{1}=0$ to satisfy $\mathscr{L}_{7} . r_{v}=0$ satisfies $\mathscr{L}_{5}$ with equality, so $\mathscr{L}_{9}$ is also satisfied. $r_{c}>r_{v}=0$ satisfies $\mathscr{L}_{6}$ with inequality, so $\lambda_{4}=0$ to satisfy $\mathscr{L}_{10}$. We now break this case into two subcases: $r_{c}=n_{c}$ or $r_{c}<n_{c}$.

Subcase 2a: $r_{c}=n_{c}$ and $r_{v}=0 \quad$ In this subcase, $r_{c}=n_{c}$ satisfies $\mathscr{L}_{4}$ with equality which also satisfies $\mathscr{L}_{8}$. Thus, $\mathscr{L}_{1}$ becomes

$$
\begin{array}{r}
-p_{v}^{\prime}(0)+p_{m}^{\prime}\left(n_{v}\right)-c+\lambda_{3}=0 \\
\Longrightarrow \lambda_{3}=p_{v}^{\prime}(0)-p_{m}^{\prime}\left(n_{v}\right)+c \geq 0 \\
\Longrightarrow p_{v}^{\prime}(0)+c \geq p_{m}^{\prime}\left(n_{v}\right)
\end{array}
$$

Similarly, $\mathscr{L}_{2}$ becomes

$$
\begin{array}{r}
p_{m}\left(n_{v}\right)^{\prime}-c-\lambda_{2}=0 \\
\Longrightarrow \lambda_{2}=p_{m}^{\prime}\left(n_{v}\right)-c \geq 0 \\
\Longrightarrow p_{m}^{\prime}\left(n_{v}\right) \geq c
\end{array}
$$

The two inequalities implied by KKT conditions $\mathscr{L}_{3}$ and $\mathscr{L}_{4}$ may apply in some cases but are also likely to be violated in other situations. The requirement that follows from KKT condition $\mathscr{L}_{3}$-namely $p_{v}^{\prime}(0)-p_{m}^{\prime}\left(n_{v}\right)+c \geq 0$-requires that the marginal penalty for violations moving from zero violations plus the marginal cost of monitoring is greater than the marginal penalty for missingness at $n_{v}$. Because many settings involve fairly light penalties for small numbers of violations, $p_{v}^{\prime}(0)$ may often be quite small (possibly near zero).

If $p_{v}^{\prime}(0)$ is indeed negligible, then these two inequalities nearly rule each other out: if the marginal cost of monitor exceeds the marginal penalty for missingness (at $n_{v}$ ), then $\mathscr{L}_{1}$ is satisfied but $\mathscr{L}_{2}$ is 
violated (and vice versa). When $p_{m}^{\prime}\left(n_{v}\right)>c$ (sufficiently steep penalization for missingness near $n_{v}$ either aggressive penalties or many violations) and when $p_{v}^{\prime}(0)$ (sufficiently steep penalization for the first violations) that this subcase is feasible.

We note that, in the case of AQS monitors, $p_{v}^{\prime}(0)$ and $c$ are both quite small; $p_{m}^{\prime}\left(n_{v}\right)$ is likely sufficiently large to make this case infeasible.

Subcase 2b: $n_{c}>r_{c}>0$ and $r_{v}=0 \quad$ In this subcase, $r_{c}<n_{c}$ satisfies $\mathscr{L}_{4}$ with inequality, so $\lambda_{2}=0$ to satisfy $\mathscr{L}_{8}$. Thus, $\mathscr{L}_{2}$ implies

$$
p_{m}^{\prime}\left(N-r_{c}\right)=c
$$

Therefore, $\mathscr{L}_{1}$ becomes

$$
\begin{array}{r}
-p_{v}^{\prime}(0)+p_{m}^{\prime}\left(N-r_{c}\right)-c+\lambda_{3}=0 \\
\Longrightarrow \lambda_{3}=p_{v}^{\prime}(0) \geq 0
\end{array}
$$

This subcase—no reporting of violations and imperfect reporting of compliance—is feasible for many settings.

\section{Subcase 3: No reporting of compliance $\left(r_{v}>r_{c}=0\right)$}

It is straightforward that this subcase would be infeasible: there are more penalties for reporting violations than compliance. $r_{c}=0$ satisfies $\mathscr{L}_{4}$ with inequality, implying $\lambda_{2}=0$ to satisfy $\mathscr{L}_{8} \cdot r_{c}=0$ satisfies $\mathscr{L}_{6}$ and $\mathscr{L}_{10}$ with equality. $r_{v}>0$ satisfies $\mathscr{L}_{5}$ with inequality, implying $\lambda_{3}=0$ to satisfy $\mathscr{L}_{9}$. As before, let us break this case into two subcases: $r_{v}=n_{v}$ and $r_{v}<n_{v}$.

Case 3a: $r_{v}=n_{v}$ and $r_{c}=0$ Here, $r_{v}=n_{v}$ satisfies $\mathscr{L}_{3}$ and $\mathscr{L}_{7}$ with equality. Therefore, $\mathscr{L}_{1}$ becomes

$$
\begin{array}{r}
-p_{v}^{\prime}\left(n_{v}\right)+p_{m}^{\prime}\left(n_{c}\right)-c-\lambda_{1}=0 \\
\Longrightarrow \lambda_{1}=p_{v}^{\prime}\left(n_{v}\right)-p_{m}^{\prime}\left(n_{c}\right)+c \geq 0 \\
\Longrightarrow p_{v}^{\prime}\left(n_{v}\right)+c \geq p_{m}^{\prime}\left(n_{c}\right)
\end{array}
$$

And $\mathscr{L}_{2}$ becomes

$$
\begin{array}{r}
p_{m}^{\prime}\left(n_{c}\right)-c+\lambda_{4}=0 \\
\Longrightarrow \lambda_{4}=c-p_{m}^{\prime}\left(n_{c}\right) \geq 0 \\
\Longrightarrow c \geq p_{m}^{\prime}\left(n_{c}\right)
\end{array}
$$

This second requirement, i.e., $c \geq p_{m}^{\prime}\left(n_{c}\right)$, is likely infeasible in most settings, where compliance is at least as common as violations. In such settings, one might expect that when half of the planned 
observations are missing, the marginal penalty levied for another missing observation would exceed the marginal cost of monitoring.

The first inequality $\left(p_{v}^{\prime}\left(n_{v}\right)+c \geq p_{m}^{\prime}\left(n_{c}\right)\right)$ is satisfied when the second is. Further, this case is infeasible as it is dominated by reporting compliance. Consider the scenario where the monitor would report $r^{*}$ violations and 0 compliant observations. Now switch to $r^{*}$ compliant violations and 0 violations.(Or as many compliant observations as possible before $n_{c}$ binds and then fill in with reports of violations.) Both scenarios generate the same costs and the same penalties for missingness. However, the $r^{*}$ violations generate a violations-based penalty of $p_{v}\left(r^{*}\right)$, while the scenarios with $r^{*}$ compliant observations generates zero penalty. Thus, reporting $r^{*}$ compliant observations dominates reporting $r^{*}$ violation observations. In other words: This subcase is infeasible.

Subcase 3b: $n_{v}>r_{v}>0$ and $r_{c}=0$ Here, $r_{v}<n_{v}$ satisfies $\mathscr{L}_{3}$ with inequality, requiring $\lambda_{1}=0$ to satisfy $\mathscr{L}_{7}$. Consequently, $\mathscr{L}_{1}$ becomes

$$
-p_{v}^{\prime}\left(r_{v}\right)+p_{m}^{\prime}\left(N-r_{v}\right)-c=0
$$

And, $\mathscr{L}_{2}$ becomes

$$
p_{m}^{\prime}\left(N-r_{v}\right)-c+\lambda_{4}=0
$$

Jointly, these two conditions imply $\lambda_{4}=-p_{v}^{\prime}\left(r_{v}\right)$, which is infeasible, as it implies $\lambda_{4}<0$. Recall that we assume $p_{v}^{\prime}>0$ (i.e., strictly increasing penalties for violations). This subcase is infeasible.

\section{Case 4: Non-zero reporting of violations and compliance $\left(r_{v}>0\right.$ and $\left.r_{c}>0\right)$}

In this case, $r_{v}>0$ satisfies $\mathscr{L}_{5}$ with inequality, implying $\lambda_{3}=0$ to satisfy $\mathscr{L}_{9} . r_{c}>0$ satisfies $\mathscr{L}_{6}$ with inequality, implying $\lambda_{4}=0$ to satisfy $\mathscr{L}_{10}$. We now break this case into four subcases based upon whether $r_{v}<n_{v}$ or $r_{v}=n_{v}$ and whether $r_{c}<n_{c}$ or $r_{c}=n_{c}$.

Subcase 4a: $r_{v}=n_{v}(>0) r_{c}=n_{c}(>0) \quad$ Here, $r_{v}=n_{v}$ satisfies $\mathscr{L}_{3}$ and $\mathscr{L}_{7}$ with equality. $r_{c}=n_{c}$ satisfies $\mathscr{L}_{4}$ and $\mathscr{L}_{8}$ with equality. $\mathscr{L}_{1}$ becomes

$$
\begin{array}{r}
-p_{v}^{\prime}\left(n_{v}\right)+p_{m}^{\prime}(0)-c-\lambda_{1}=0 \\
\Longrightarrow \lambda_{1}=-p_{v}^{\prime}\left(n_{v}\right)+p_{m}^{\prime}(0)-c \geq 0 \\
\Longrightarrow p_{m}^{\prime}(0) \geq c+p_{v}^{\prime}\left(n_{v}\right)
\end{array}
$$


$\mathscr{L}_{2}$ becomes

$$
\begin{aligned}
p_{m}^{\prime}(0)-c-\lambda_{2} & =0 \\
\Longrightarrow \lambda_{2}=p_{m}^{\prime}(0)-c & \geq 0 \\
\Longrightarrow p_{m}^{\prime}(0) & \geq c
\end{aligned}
$$

The inequality implied by $\mathscr{L}_{1}$, i.e., $p_{m}^{\prime}(0) \geq c+p_{v}^{\prime}\left(n_{v}\right)$, requires that the marginal penalty for missingness at zero missing observations is larger than the sum of marginal monitoring costs and the marginal penalty for violations at $n_{v}$. Thus, for complete, honest reporting to be feasible, the marginal cost of missing any observations must overwhelm marginal operating costs and penalties from additional violations when all violations have been reported. Unless $n_{v} \approx 0$, the assumed convexity of $p_{m}$ and $p_{v}$ likely rules out this possibility.

Subcase 4b: $n_{v}>r_{v}>0$ and $r_{c}=n_{c}>0$ Here, $n_{v}>r_{v}>0$ satisfies $\mathscr{L}_{3}$ with inequality, requiring $\lambda_{1}=0$ to satisfy $\mathscr{L}_{7} . r_{c}=n_{c}$ satisfies $\mathscr{L}_{4}$ and $\mathscr{L}_{8}$ with equality. $\mathscr{L}_{1}$ becomes

$$
\begin{aligned}
& -p_{v}^{\prime}\left(r_{v}\right)+p_{m}^{\prime}\left(n_{v}-r_{v}\right)-c=0 \\
& \Longrightarrow p_{m}^{\prime}\left(n_{v}-r_{v}\right)=p_{v}^{\prime}\left(r_{v}\right)+c
\end{aligned}
$$

$\mathscr{L}_{2}$ becomes

$$
\begin{aligned}
p_{m}^{\prime}\left(n_{v}-r_{v}\right)-c-\lambda_{2} & =0 \\
\Longrightarrow \lambda_{2}=p_{m}^{\prime}\left(n_{v}-r_{v}\right)-c & \geq 0 \\
\Longrightarrow p_{m}^{\prime}\left(n_{v}-r_{v}\right) & \geq c
\end{aligned}
$$

These conditions are feasible for most settings.

Subcase 4c: $r_{v}=n_{v}>0$ and $n_{c}>r_{c}>0$ Here, $r_{v}=n_{v}$ satisfies $\mathscr{L}_{3}$ and $\mathscr{L}_{7}$ with equality. $r_{c}<n_{c}$ satisfies $\mathscr{L}_{4}$ with inequality, which requires $\lambda_{2}=0$ to satisfy $\mathscr{L}_{8} \cdot \mathscr{L}_{1}$ becomes

$$
-p_{v}^{\prime}\left(n_{v}\right)+p_{m}^{\prime}\left(n_{c}-r_{c}\right)-c-\lambda_{1}=0
$$

$\mathscr{L}_{2}$ becomes

$$
p_{m}^{\prime}\left(n_{c}-r_{c}\right)-c=0
$$

Jointly, these two conditions imply $\lambda_{1}=-p_{v}^{\prime}\left(n_{v}\right)<0$, which is infeasible.

Subcase 4d: $n_{v}>r_{v}>0$ and $n_{c}>r_{c}>0 \quad$ Finally, $r_{v}<n_{v}$ satisfies $\mathscr{L}_{3}$ with inequality, requiring $\lambda_{1}=0$ to satisfy $\mathscr{L}_{7} . r_{c}<n_{c}$ satisfies $\mathscr{L}_{4}$ with inequality, requiring $\lambda_{2}=0$ to satisfy $\mathscr{L}_{8} . r_{v}>0$ satisfies $\mathscr{L}_{5}$ 
with inequality, requiring $\lambda_{3}=0$ to satisfy $\mathscr{L}_{9} . r_{c}>0$ satisfies $\mathscr{L}_{6}$ with inequality, requiring $\lambda_{4}=0$ to satisfy $\mathscr{L}_{10} \cdot \mathscr{L}_{1}$ becomes

$$
-p_{v}^{\prime}\left(r_{v}\right)+p_{m}^{\prime}\left(N-r_{v}-r_{c}\right)-c=0
$$

$\mathscr{L}_{2}$ becomes

$$
p_{m}^{\prime}\left(N-r_{v}-r_{c}\right)-c=0
$$

Jointly, $\mathscr{L}_{1}$ and $\mathscr{L}_{2}$ imply $p_{v}^{\prime}\left(r_{v}\right)=0$, which is not feasible (By assumption: $p_{v}^{\prime \prime}>0$.)

To summarize, Table A.1 tabulates our conclusions for all the cases above.

\begin{tabular}{|c|c|c|c|c|c|}
\hline \multirow[b]{2}{*}{ Case } & \multirow[b]{2}{*}{ Reporting } & \multicolumn{2}{|c|}{ Pct. of observations reported } & \multirow[b]{2}{*}{ Feasibility } & \multirow[b]{2}{*}{ Constraint(s) } \\
\hline & & Violations & Compliance & & \\
\hline 1 & $r_{v}=r_{c}=0$ & $0 \%$ & $0 \%$ & Unlikely & $c \geq p_{m}^{\prime}(N)$ \\
\hline $2 \mathrm{a}$ & $r_{v}=0$ and $r_{c}=n_{c}$ & $0 \%$ & $100 \%$ & Feasible & $\begin{aligned} p_{v}^{\prime}(0)+c & \geq p_{m}^{\prime}\left(n_{v}\right) \\
p_{m}^{\prime}\left(n_{v}\right) & \geq c\end{aligned}$ \\
\hline $2 b$ & $r_{v}=0$ and $n_{c}>r_{c}>0$ & $0 \%$ & $\in(0 \%, 100 \%)$ & Feasible & $p_{m}^{\prime}\left(N-r_{c}\right)=c$ \\
\hline $3 a$ & $r_{v}=n_{v}$ and $r_{c}=0$ & $100 \%$ & $0 \%$ & Infeasible & $c \geq p_{m}^{\prime}\left(n_{c}\right)$ \\
\hline $3 b$ & $n_{v}>r_{v}>0$ and $r_{c}=0$ & $\in(0 \%, 100 \%)$ & $0 \%$ & Infeasible & $\lambda_{4}=-p_{v}^{\prime}\left(r_{v}\right)<0$ \\
\hline $4 a$ & $r_{v}=n_{v}$ and $r_{c}=n_{c}$ & $100 \%$ & $100 \%$ & Unlikely & $p_{m}^{\prime}(0) \geq c+p_{v}^{\prime}\left(n_{v}\right)$ \\
\hline $4 b$ & $n_{v}>r_{v}>0$ and $r_{c}=n_{c}$ & $\in(0 \%, 100 \%)$ & $100 \%$ & Feasible & $p_{m}^{\prime}\left(n_{v}-r_{v}\right) \geq c$ \\
\hline $4 \mathrm{c}$ & $r_{v}=n_{v}$ and $n_{c}>r_{c}>0$ & $100 \%$ & $\in(0 \%, 100 \%)$ & Infeasible & $\lambda_{1}=-p_{v}^{\prime}\left(n_{v}\right)<0$ \\
\hline $4 d$ & $n_{v}>r_{v}>0$ and $n_{c}>r_{c}>0$ & $\in(0 \%, 100 \%)$ & $\in(0 \%, 100 \%)$ & Infeasible & $p_{v}^{\prime}\left(r_{v}\right)=0$ \\
\hline
\end{tabular}

\section{Table A.1: Summary of Possible Scenarios}


Appendix B. Additional Figures and Tables 
Figure B.1. Monitoring Data Completeness Goals

Table 6-4 Completeness Goals for Ambient Air Monitoring Data

\begin{tabular}{|c|c|c|c|c|c|c|}
\hline & \multicolumn{6}{|c|}{ Completeness Goals and Associated Standards (highlighted) } \\
\hline Pollutants & 1-hour & 3-hour & 8-hour & 24-hour & Quarterly & Annual \\
\hline $\mathrm{CO}$ & $45,1 \mathrm{~min}$. values & & $\begin{array}{l}75 \% \text { of } \\
\text { hourly values }\end{array}$ & $\begin{array}{l}75 \% \text { of } \\
\text { hourly values }\end{array}$ & & $\begin{array}{l}75 \% \text { of hourly values } \\
\text { per quarter }\end{array}$ \\
\hline $\mathrm{O}_{3}$ & 45,1 min. values & & $\begin{array}{l}75 \% \text { of } \\
\text { hourly values }\end{array}$ & $\begin{array}{l}13 \text { of } 178- \\
\text { hour periods. }\end{array}$ & & $\begin{array}{l}75 \% \text { of days within } \\
\text { season }^{1}\end{array}$ \\
\hline $\mathrm{SO}_{2}$ & $45,1 \mathrm{~min}$. values & $\begin{array}{l}\text { All } 3 \text { hours } \\
75 \% \text { complete }\end{array}$ & & $\begin{array}{l}75 \% \text { of } \\
\text { hourly values }\end{array}$ & & $\begin{array}{l}75 \% \text { of hourly values } \\
\text { per quarter }\end{array}$ \\
\hline $\mathrm{NO}_{2}$ & $45,1 \mathrm{~min}$. values & & & & & $\begin{array}{l}75 \% \text { of hourly values } \\
\text { per quarter }\end{array}$ \\
\hline $\mathrm{PM}_{10}$ Cont & $45,1 \mathrm{~min}$. values & & & 18 Hours & & \\
\hline $\mathrm{PM}_{2.5}$ Cont. & $45,1 \mathrm{~min}$. values & & & 18 Hours & & \\
\hline $\begin{array}{l}\mathrm{PM}_{10} \\
\text { Manual }\end{array}$ & & & & 23 Hours** & & \\
\hline $\begin{array}{l}\text { PM2s } \\
\text { Manual }\end{array}$ & & & & 23 hours & $75 \%$ of samples & \\
\hline $\mathrm{Pb}$ & & & & 23 Hours & $\begin{array}{l}3 \text { mo avg }>75 \% \text { of } \\
\text { monthly means }\end{array}$ & \\
\hline PAMS & & & & 23 Hours & & \\
\hline NATTS & & & & 23 Hours & & \\
\hline $\mathrm{CSN}$ & & & & 23 Hours & & \\
\hline
\end{tabular}

** not defined in CFR

$1=$ For ozone the requirements are met for a 3-year period at a site if valid daily maximum 8 -hour average $\mathrm{O}_{3}$

concentrations are available for at least $90 \%$ of the days within the $\mathrm{O}_{3}$ monitoring season, on average, for the $3-\mathrm{year}$

period, with a minimum of at least $75 \%$ of the days within the $\mathrm{O}_{3}$ monitoring season in any one year

Notes: Sourced from U.S. EPA. Quality Assurance Handbook for Air Pollution Measurement Systems Volume II Ambient Air Quality Monitoring Program. Vol. 2. EPA-454/B-13-003 (2013). From Section 6.0, page 66: "The data cells highlighted in Table 6-4 refer to the standards that apply to the specific pollutant. Even though a highlighted cell lists the completeness requirement, CFR provides additional detail, in some cases, on how a design value might be calculated with less data than the stated requirement. Therefore, the information provided in Table 6-4 should be considered the initial completeness goal." 
Figure B.2. Air Pollution Monitoring Site Example

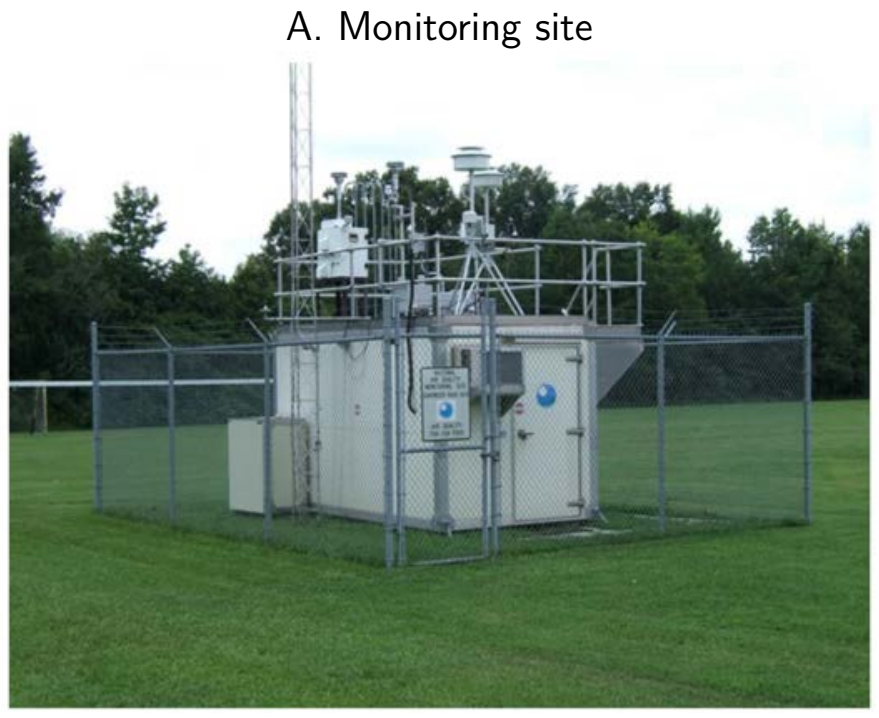

B. Shelter Design
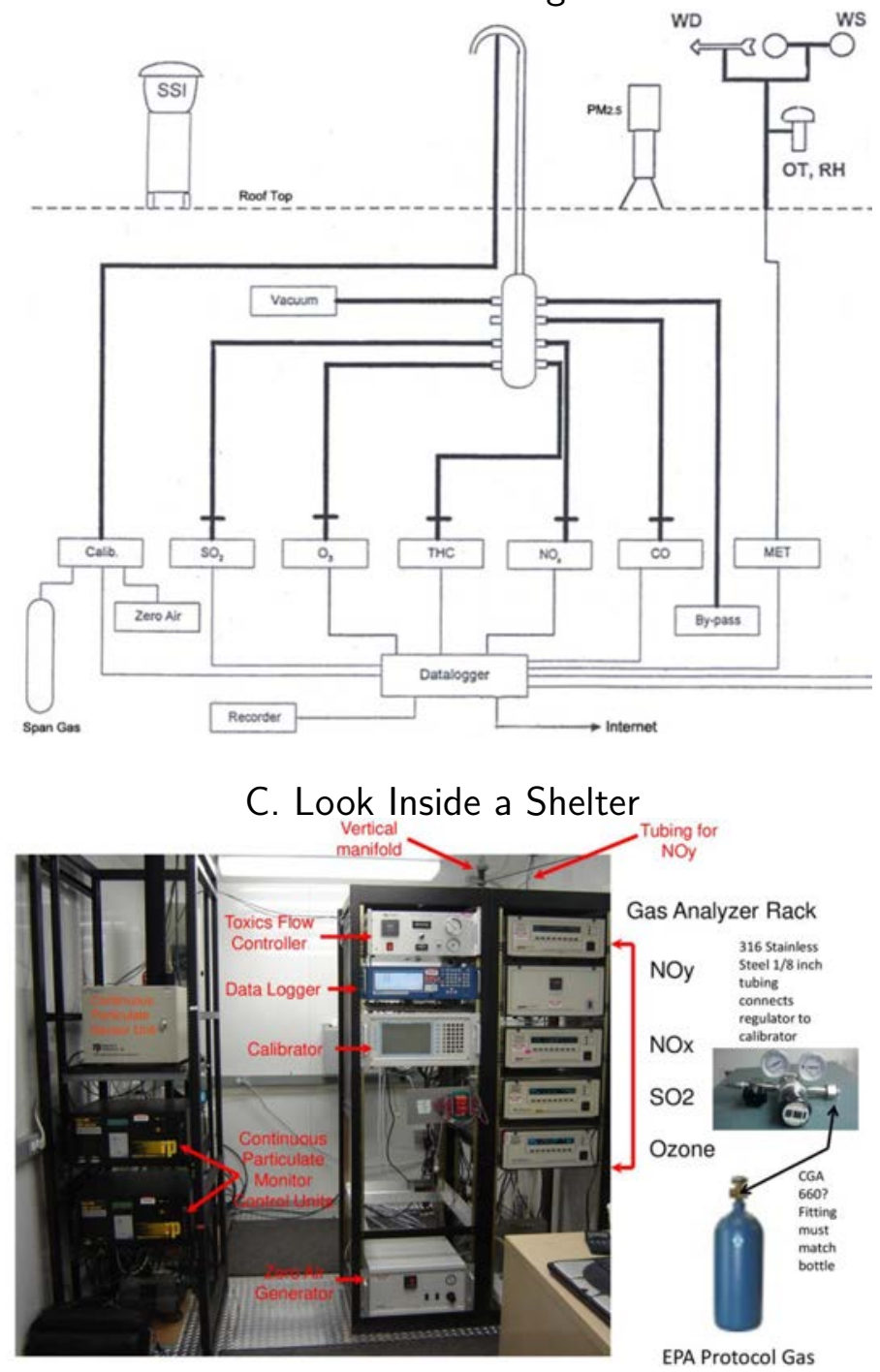

Sources: U.S. EPA (panel A), California Resources Board (panel B), and Glenn Gehring (panel C). 
Figure B.3. Distribution of Forecasted AQI on Alert Days

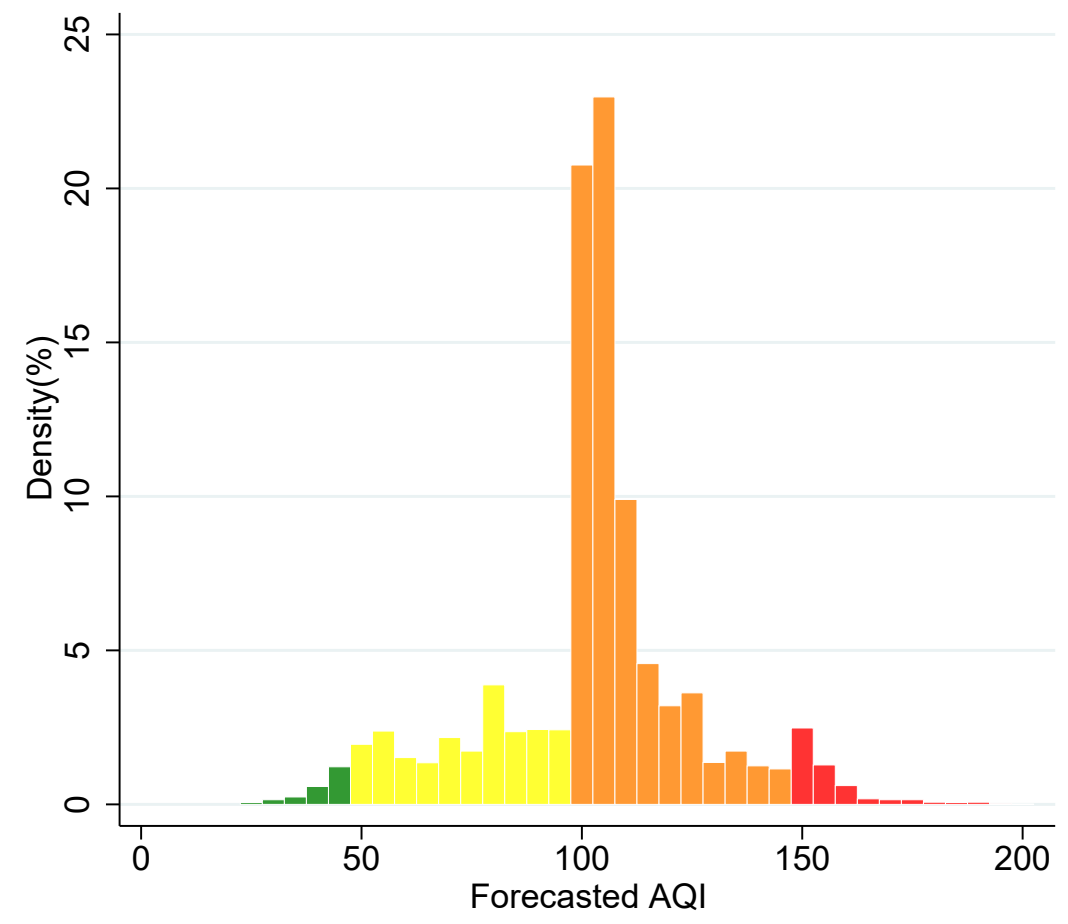

Notes: Distribution of forecasted Air Quality Index on days with pollution alerts issued.

Figure B.4. Distribution of $p$-values: Robustness

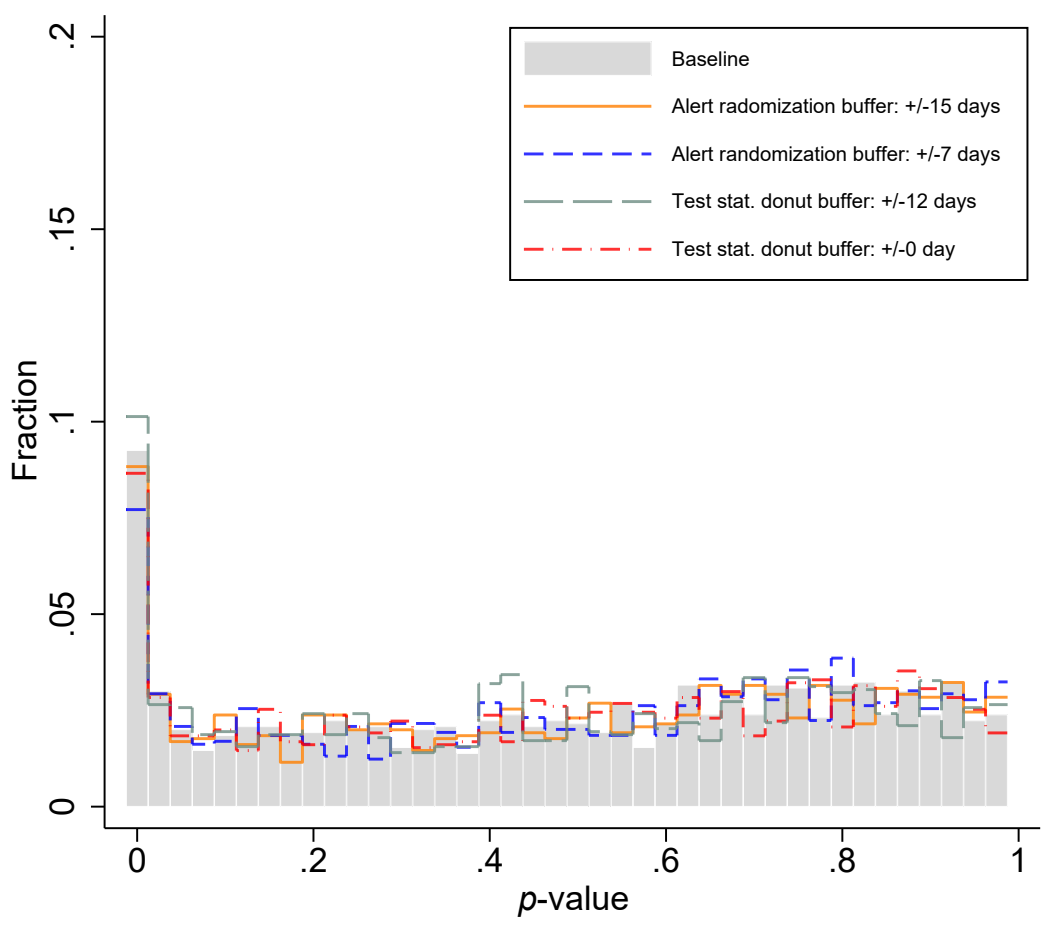

Notes: Robustness checks of panel A, Figure 2. See Section 4 of the paper for details. 
Figure B.5. Robustness: "Interesting" Monitors $(\triangle)$ and Other Monitors $(\bigcirc)$ Separation with the Sharpened Test Statistic
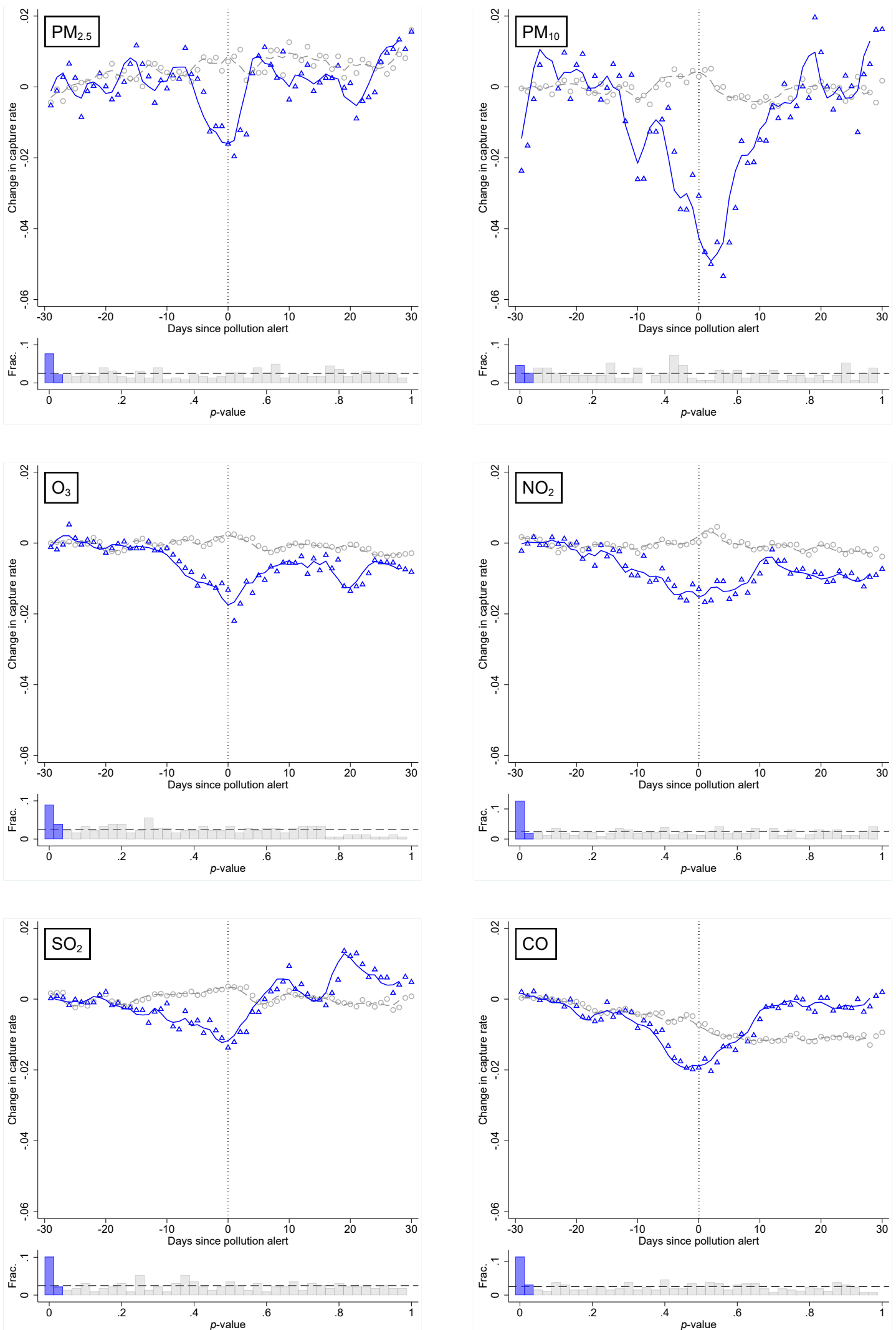

Notes: This graph shows mean monitoring capture rate for "interesting" monitors (those with $p$-value<0.05) and other monitors. The sharpened test rejects the null if the capture rate around time zero is lower than both the pre-period and the post-period. See Section 4.1 for more details. Data are demeaned by the average capture rate across the first ten event days. Fitted lines show three-day moving averages of point estimates. Each panel corresponds to one pollutant. Histograms show the distributions of $p$-values for the corresponding pollutant monitors. 
Figure B.6. Distributions of Daily Observed and Nearby-Monitor-Predicted Concentration, Non-PM 2.5 Monitors A. $\mathrm{PM}_{10}$ monitors, "interesting" (left) and "non-interesting" (right)
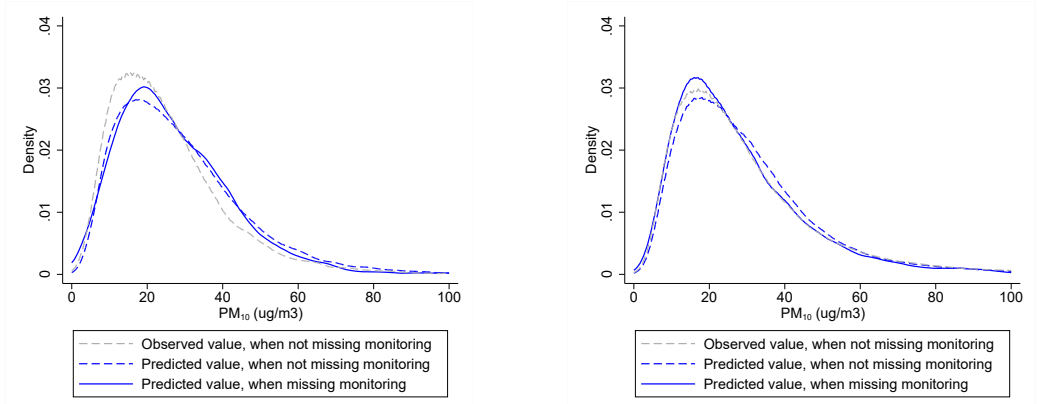

B. $\mathrm{O}_{3}$ monitors, "interesting" (left) and "non-interesting" (right)
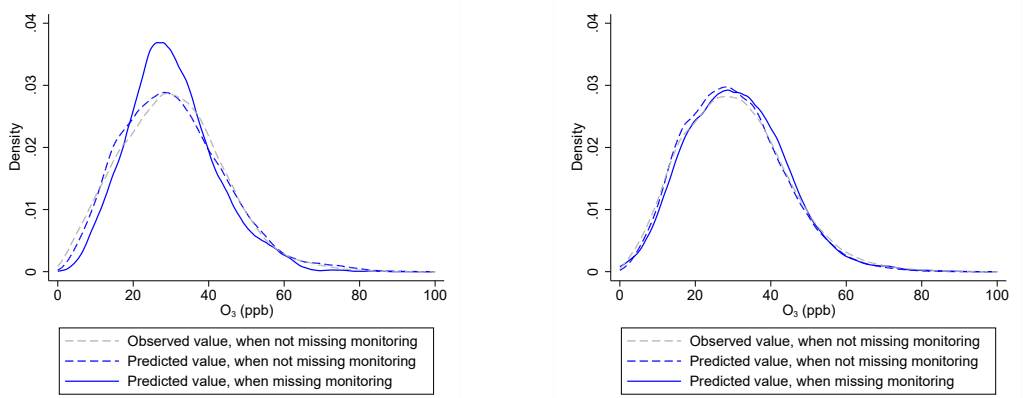

C. $\mathrm{NO}_{2}$ monitors, "interesting" (left) and "non-interesting" (right)
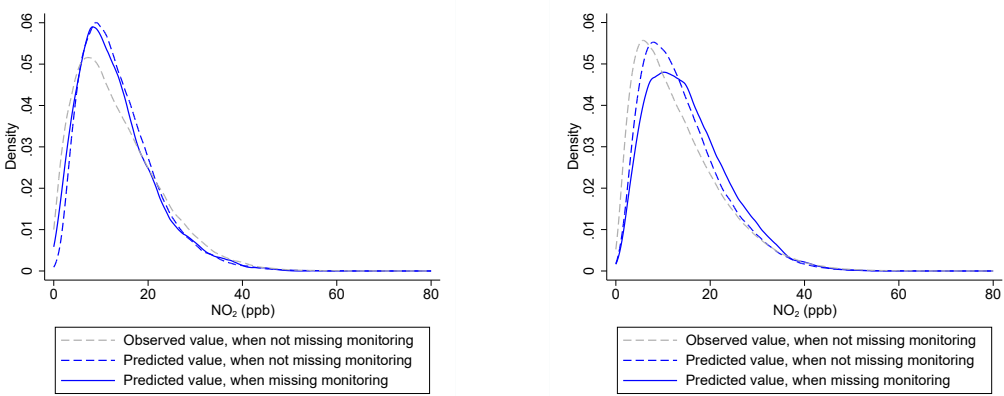

D. $\mathrm{SO}_{2}$ monitors, "interesting" (left) and "non-interesting" (right)
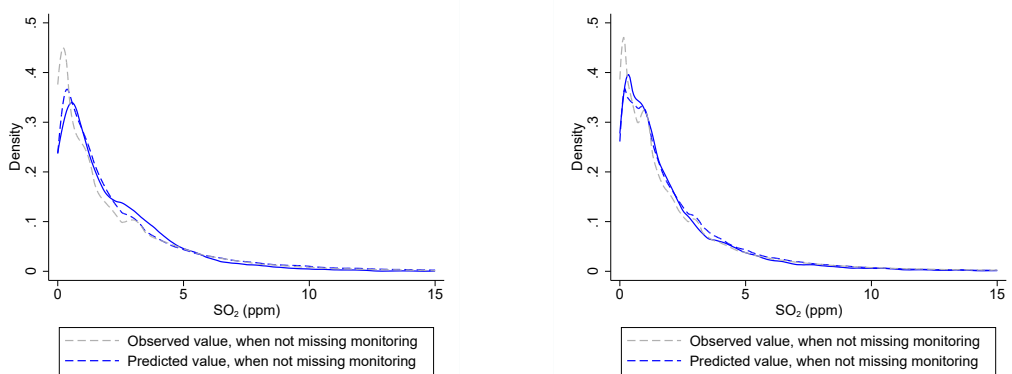
value, when not missing monitoring

E. CO monitors, "interesting" (left) and "non-interesting" (right)
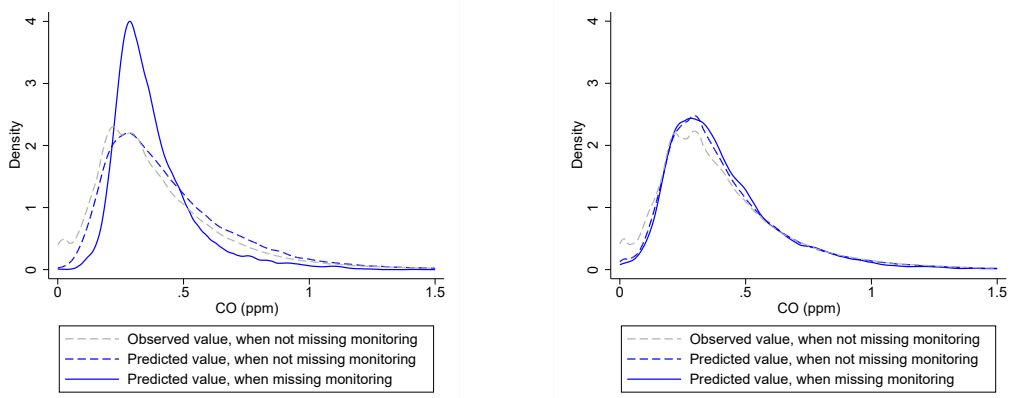

Notes: Repetition of panel A, Figure 9 with other pollutant monitors. See Section 5 of the paper for details. 